TOPOLOGY CONTROL VECTOR BASED FORWARDING ALGORITHM FOR UNDERWATER ACOUSTIC NETWORKS

A THESIS SUBMITTED TO

THE GRADUATE SCHOOL OF NATURAL AND APPLIED SCIENCES

$\mathrm{OF}$

MIDDLE EAST TECHNICAL UNIVERSITY

BY

İLKAY YAZGI

IN PARTIAL FULFILLMENT OF THE REQUIREMENTS

FOR

THE DEGREE OF MASTER OF SCIENCE

IN

ELECTRICAL AND ELECTRONICS ENGINEERING

DECEMBER 2015 

Approval of the thesis:

\section{TOPOLOGY CONTROL VECTOR BASED FORWARDING ALGORITHM FOR UNDERWATER ACOUSTIC NETWORKS}

submitted by IIlkay Yazgı in partial fulfillment of the requirements for the degree of Master of Science in Electrical and Electronics Engineering Department, Middle East Technical University by,

Prof. Dr. Gülbin Dural Ünver

Dean, Graduate School of Natural and Applied Sciences

Prof. Dr. Gönül Turhan Sayan

Head of Department, Electrical and Electronics Engineering

Prof. Dr. Buyurman Baykal

Supervisor, Electrical and Electronics Eng. Dept., METU

\section{Examining Committee Members:}

Prof. Dr. Ali Özgür Y1lmaz

Electrical and Electronics Engineering Dept., METU

Prof. Dr. Buyurman Baykal

Electrical and Electronics Engineering Dept., METU

Assoc. Prof. Dr. Cüneyt F. Bazlamaçcı

Electrical and Electronics Engineering Dept., METU

Assoc. Prof. Dr. Senan Ece Güran Schmidt

Electrical and Electronics Engineering Dept., METU

Prof. Dr. Bülent Tavlı

Electrical and Electronics Engineering Dept., TOBB-UET

Date:

$09 / 12 / 2015$ 
I hereby declare that all information in this document has been obtained and presented in accordance with academic rules and ethical conduct. I also declare that, as required by these rules and conduct, $I$ have fully cited and referenced all material and results that are not original to this work.

Name, Last name $\quad$ : Illkay Yazg1

Signature 


\title{
ABSTRACT \\ TOPOLOGY CONTROL VECTOR BASED FORWARDING ALGORITHM FOR UNDERWATER ACOUSTIC NETWORKS
}

\author{
Yazg1, İlkay \\ M.S., Department of Electrical and Electronics Engineering \\ Supervisor: Prof. Dr. Buyurman Baykal
}

December 2015, 86 pages

The communication range of underwater acoustic sensor networks (UASN) is limited by the underwater environment. Acoustic networks with huge number of sensors may have long communication range with appropriate protocols in literature. On the other hand, especially, the networks including small number of nodes have communication problems for long ranges. To challenge this problem, topology control in underwater acoustic networks is a promising solution. In this study, a novel approach, Topology Control Vector Based Forwarding Protocol (TC-VBF) is introduced and developed. In the TC-VBF, when the communication with source node and target node becomes a problem due to range, the actor node will help the packet transfer. The actor node is moving to the mid-point of the distance between master node and sink node. When the packet transfer is successfully finished, the actor node moves back to previous position. Existing MAC, transport and routing protocols and topology control algorithms are classified and analyzed. A novel algorithm, TC-VBF, is presented and evaluated comparatively with alternative routing protocols. It shows better performance for reliable data transfer and long communication range.

Keywords: Underwater Acoustic Networks, Transmission Range, Topology Control, Topology Control Vector Based Forwarding (TC-VBF) 


\title{
SUALTI AKUSTIK AĞLAR İĊIN TOPOLOJİ KONTROLLÜ VEKTÖR TABANLI YÖNLENDIRME ALGORITMASI
}

\author{
Yazg1, İlkay \\ Yüksek Lisans, Elektrik ve Elektronik Mühendisliği Bölümü \\ Tez Yöneticisi: Prof. Dr. Buyurman Baykal
}

Aralık 2015, 86 sayfa

Sualtı akustik algılayıcı ağların (UASN) haberleşme mesafesi sualtı ortamının şartlarından dolayı kısıtlıdır. Çok fazla sayıda algılayıcı içeren akustik ağlar mevcut olan uygun protokollerin kullanımı ile daha geniş haberleşme mesaflerine sahip olabilirler. Öte yandan, özellikle az sayıda algılayıcı içeren akustik ağlarda uzak mesafelerde haberleşme problemdir. Bu problemin çözümü için sualtı akustik ağlarda topoloji kontrolü gelecek vadeden bir çözümdür. Bu çalışmada, yeni TCVBF yaklaşımı sunulmakta ve geliştirilmektedir. Kaynak ağ elemanı ile hedef ağ elemanı arasında haberleşme problem olmaya başladığında, TC-VBF yaklaşımında aracı ağ elemanı paket aktarımına yardım eder. Bu aracı ağ elemanı kaynak ağ elemanı ile hedef ağ elemanı arasındaki uzaklığın orta noktasına hareket eder. Paket aktarımı başarıyla tamamlandığında aracı ağ elemanı eski pozisyonuna döner. Var olan MAC, taşıma katmanı ve yönlendirme protokolleri ile topoloji kontrol algoritmaları sınıflandırılip analiz edilmektedir. Yeni TC-VBF algoritması önerilerek, alternatif yönlendirme protokolleri ile karşılaştırılmalı değerlendirilmektedir. Yeni protokolün güvenilir bilgi aktarımında ve uzun haberleşme mesafesinde daha iyi performansa sahip olduğu görülmektedir.

Anahtar Kelimeler: Sualtı Akustik Ağları, İletim Mesafesi, Topoloji Kontrolü, Topoloji Kontrollü Vektör Tabanlı Yönlendirme 
To my family 


\section{ACKNOWLEDGEMENTS}

I sincerely thank to my supervisor Prof. Dr. Buyurman Baykal for all his guidance and support throughout my study.

I would like to express my appreciation to my employer, ASELSAN.

I also thank Turkish Scientific and Technological Research Council (TÜBİTAK) for their financial support during my study.

I am grateful to my family for their love, trust and support throughout my life. 


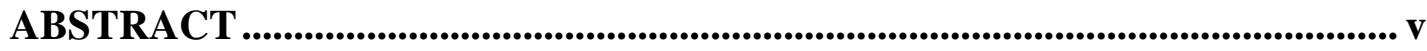

ÖZ

ACKNOWLEDGEMENTS........................................................................................... viii

TABLE OF CONTENTS................................................................................................. ix

LIST OF TABLES ...........................................................................................................

LIST OF FIGURES ...................................................................................................... xiii

LIST OF ABBREVIATIONS ..................................................................................... $\mathrm{xv}$

1. INTRODUCTION................................................................................................................. 1

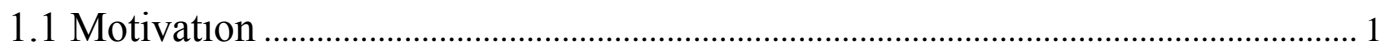

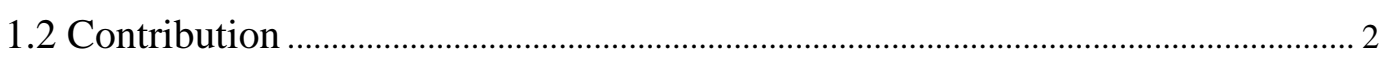

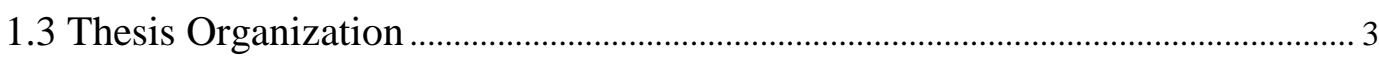

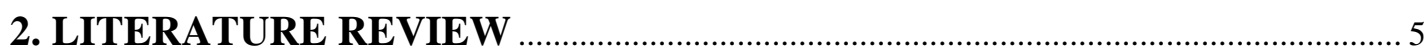

2.1 Underwater Acoustic Channel Characteristics ....................................................... 5

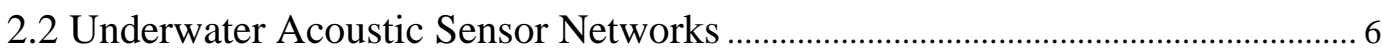

2.3 Medium Access Control (MAC) Protocols ................................................................. 8

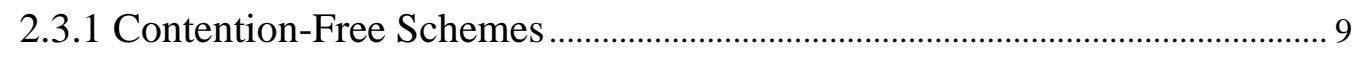

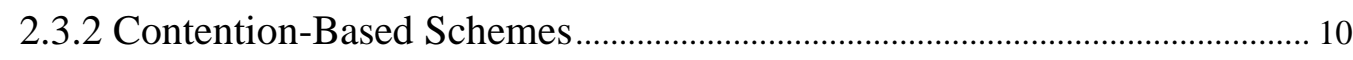

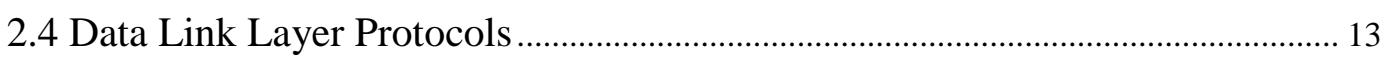

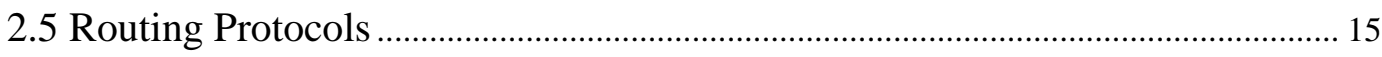

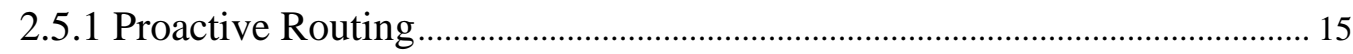

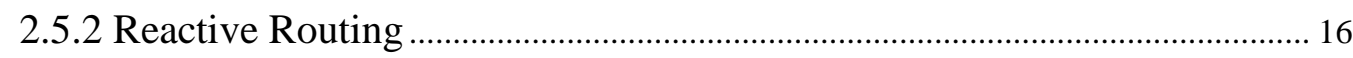

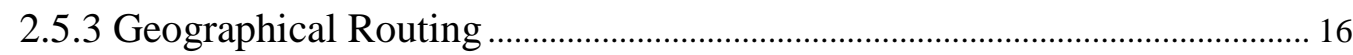

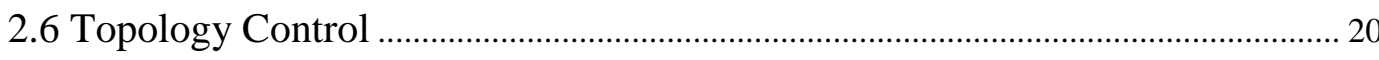


2.6.1 Definition of Topology Control ...........................................................................

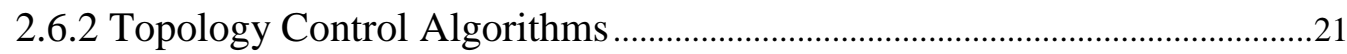

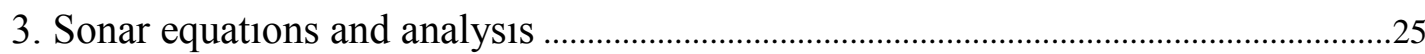

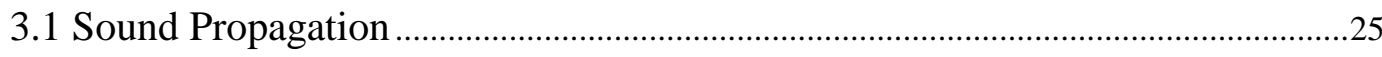

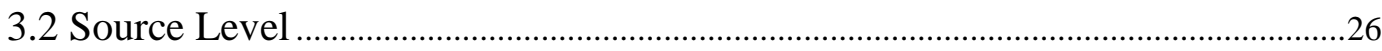

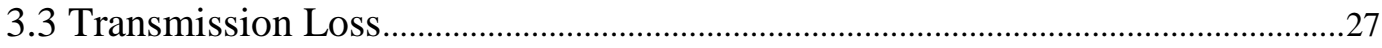

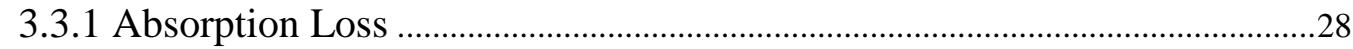

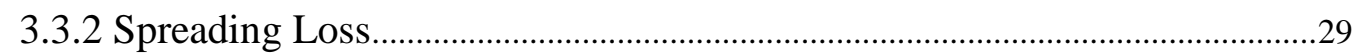

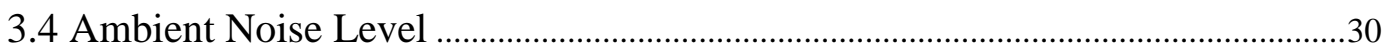

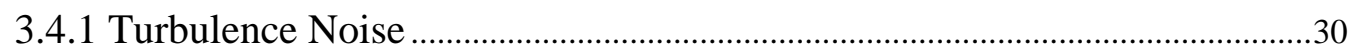

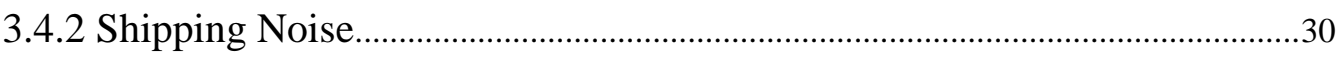

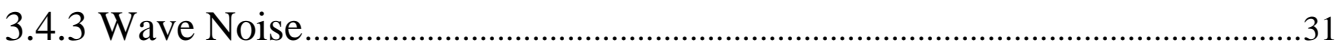

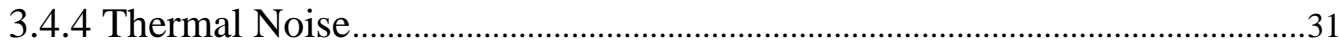

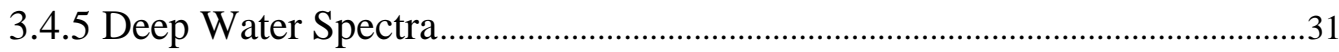

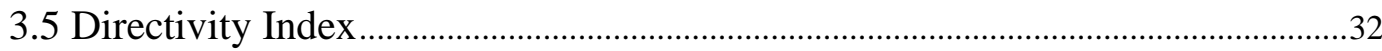

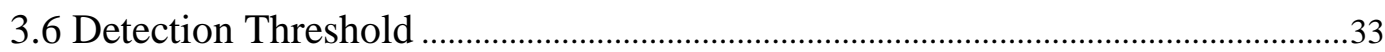

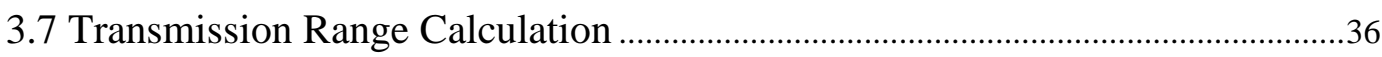

4. TOPOLOGY CONTROL VECTOR BASED FORWARDING PROTOCOL

4.1 Topology Model for Underwater Acoustic Sensor Networks .................................37

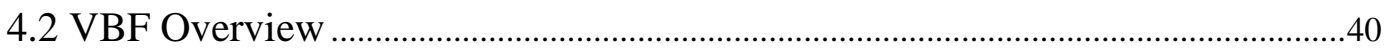

4.3 Topology Control Vector Based Forwarding Protocol ..........................................42

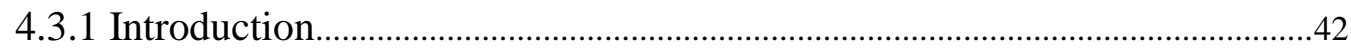

4.3.2 Protocol Description........................................................................................42

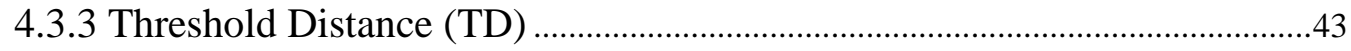

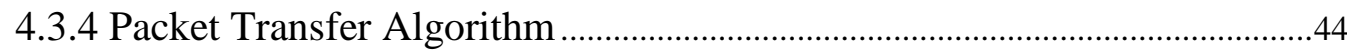

5. SIMULATION BASED PERFORMANCE EVALUATION …….......................49

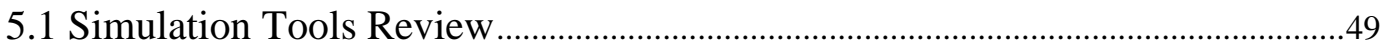

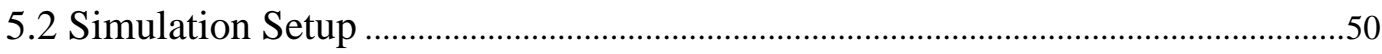


5.2.1 Point-to-Point Communication Model

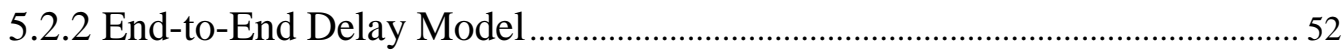

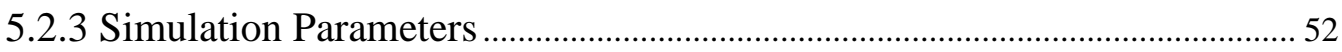

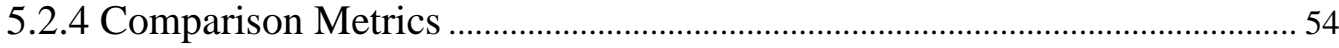

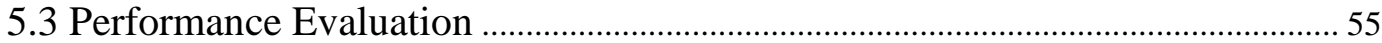

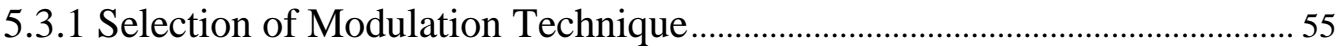

5.3.2 Performance Comparison for Topology 1 ……………………………........ 56

5.3.3 Performance Comparison for Topology 2 ……………………………........ 61

5.3.4 Performance Comparison for Long Range with $\mathrm{N}=2$ ….................................. 66

5.3.5 Performance Comparison for Long Range with $\mathrm{N}=3$ ……........................... 71

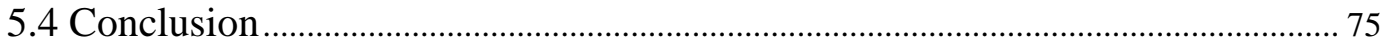

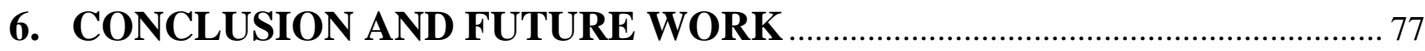

REFERENCES 


\section{LIST OF TABLES}

\section{TABLES}

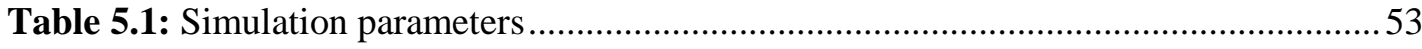

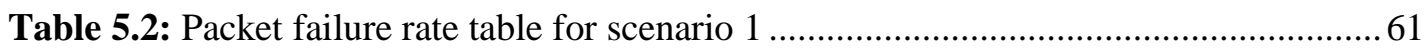

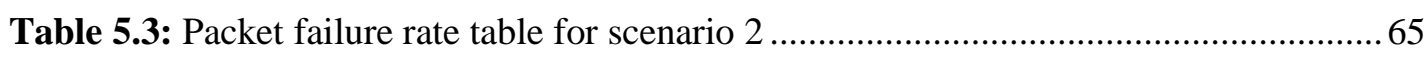

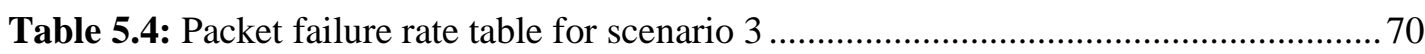

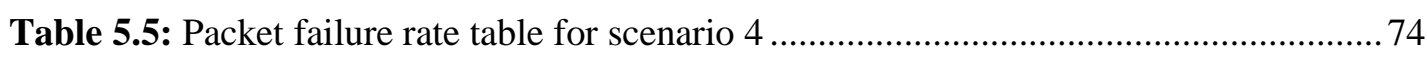




\section{LIST OF FIGURES}

\section{FIGURES}

Figure 3.1: Absorption loss coefficient characteristics, 10log $\alpha f$ [68].

Figure 3.2: The sample spectrum of the deep-sea noise showing different frequency bands

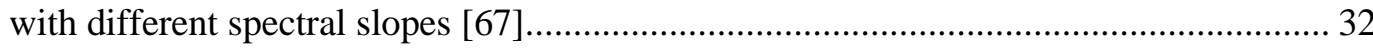

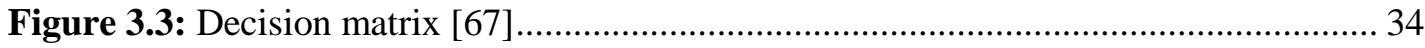

Figure 3.4: Example ROC curve of detection probability $p(D)$ against false-alarm

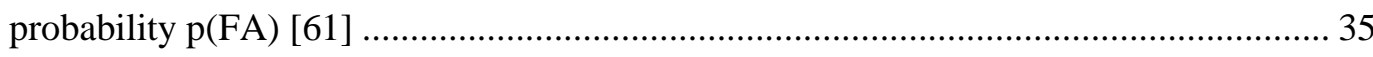

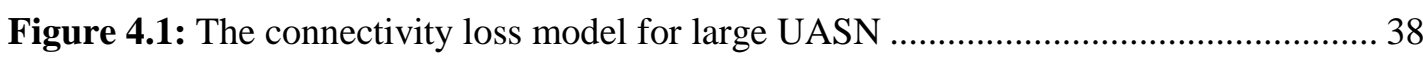

Figure 4.2: Generalized connectivity loss model for UASNs.............................................. 39

Figure 4.3: The topology control mechanism against the loss of connectivity.................... 40

Figure 4.4: The data vector and the data path for packet transfer........................................4 40

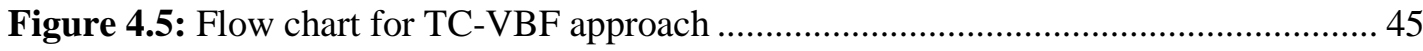

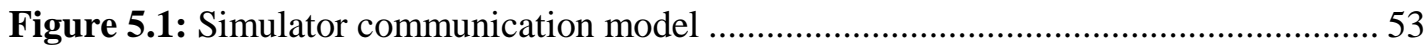

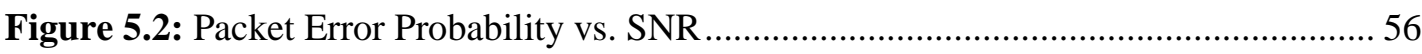

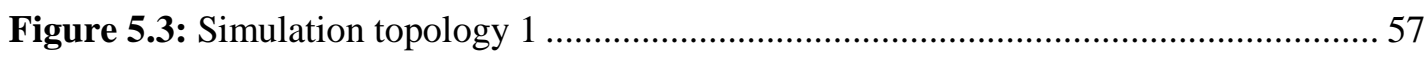

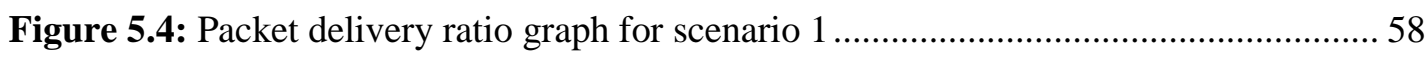

Figure 5.5: Average end-to-end delay graph for scenario 1 …........................................... 59

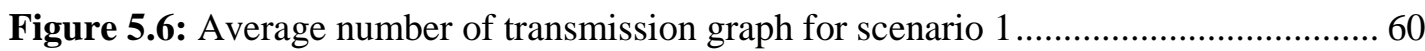

Figure 5.7: Histogram of successful transmission numbers for scenario 1.......................... 60

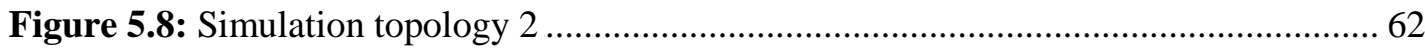

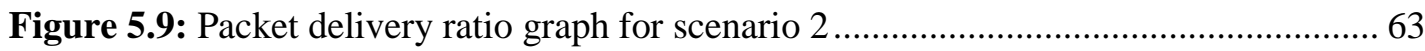

Figure 5.10: Average end-to-end delay graph for scenario 2 2............................................... 64

Figure 5.11: Average number of retransmission graph for scenario 2.................................6 64

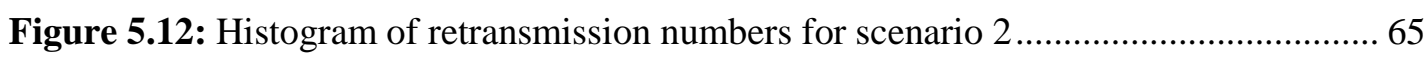

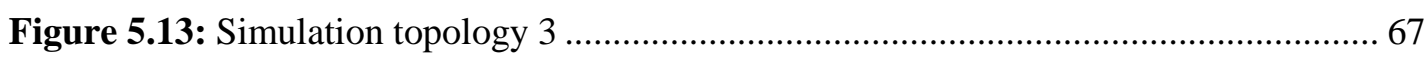

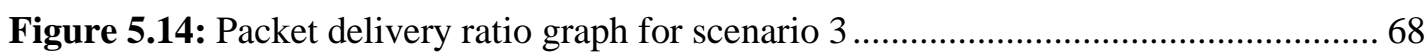

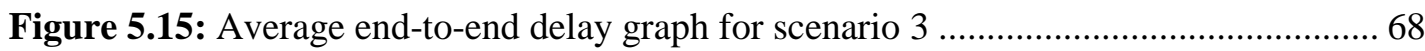

Figure 5.16: Average number of retransmission graph for scenario 3............................... 69 
Figure 5.17: Histogram of retransmission numbers for scenario 3 .70

Figure 5.18: Packet delivery ratio graph for scenario 4 ................................................... 71

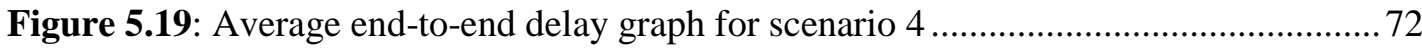

Figure 5.20: Average number of retransmission graph for scenario 4 ................................73

Figure 5.21: Histogram of retransmission numbers for scenario 4 .................................... 74 


\section{LIST OF ABBREVIATIONS}

$\begin{array}{ll}\text { ARQ } & \text { Automatic Repeat Request } \\ \text { BER } & \text { Bit Error Rate } \\ \text { BPSK } & \text { Binary Phase-Shift Keying } \\ \text { CDMA } & \text { Code Division Multiple Access } \\ \text { CSMA } & \text { Carrier Sense Multiple Access } \\ \text { DBR } & \text { Depth Based Routing } \\ \text { DI } & \text { Directivity Index } \\ \text { DT } & \text { Detection Threshold } \\ \text { FDMA } & \text { Frequency Division Multiple Access } \\ \text { FEC } & \text { Forward Error Correction } \\ \text { HH-VBF } & \text { Hop-by-Hop Vector Based Forwarding } \\ \text { MAC } & \text { Medium Access Control } \\ \text { OFDMA } & \text { Orthogonal Frequency Division Multiple Access } \\ \text { QPSK } & \text { Qaudrature Phase-Shift Keying } \\ \text { SNR } & \text { Signal-to-Noise Ratio } \\ \text { TC-VBF } & \text { Topology Control Vector Based Forwarding } \\ \text { TDMA } & \text { Time-Division Multiple Access } \\ \text { UASN } & \text { Underwater Acoustic Sensor Networks } \\ \text { UWA } & \text { Underwater Acoustic } \\ \text { VBF } & \text { Vector Based Forwarding }\end{array}$




\section{CHAPTER 1}

\section{INTRODUCTION}

Underwater research and applications are important since water covers three fourths of earth. The underwater acoustic networks are envisioned to be used in several underwater applications such as monitoring environment, prevention of disaster, navigation, military defense systems, sonar systems and seismic exploration. According to the underwater medium, these communication networks use acoustic waves instead of radio-frequency or optical waves.

\subsection{Motivation}

Although considerable number of technological developments, research and applications related to terrestrial networks has been conducted, most of those are inapplicable for underwater acoustic networks. The reasons for this problem are mainly

- high absorption rate,

- limited bandwidth,

- large propagation delay,

- limited power,

- and rapid time variation.

These challenges for underwater environment will be detailed in Chapter 2 .

When limitations and challenges of the underwater medium are considered, the communication between long distances becomes harder. The transmission range of 
underwater networks is shorter when compared to terrestrial networks. The increase in the communication range is a challenging problem for underwater acoustic networks.

ASELSAN, a leading defense system company in Turkey, possesses countermeasure products for underwater military applications, namely ZOKA $^{\mathrm{TM}}$ Acoustic Torpedo Countermeasure Decoy. It is the state of the art countermeasure used against torpedo threat. These acoustic decoys have the ability to move. It is aimed to develop communication systems for those countermeasure decoys while they are performing their missions, i.e. to add a communication ability to these decoys, in order to perform better tactical surveillance against threats.

The acoustic communication system in ASELSAN project is designed to be controlled by the master device that is located at a fixed position. Moreover, this master device is capable of taking position knowledge of decoys with the help of specific sensors. The acoustic network has a small number of nodes. The energy consumption and delay is relatively not important. However, the reliability of data transfer is critical. Furthermore, it is aimed to have a large communication range.

\subsection{Contribution}

In this thesis, we target to tackle communication range problem of underwater acoustic communication networks. Especially, the master-controlled small networks are focused. Furthermore, reliable data transfer is considered.

A new algorithm is proposed to communicate reliably with long ranges by controlling the topology. This algorithm is heuristic approach for communication range problems and there can be other possible solutions for underwater acoustic networks. Morever, the new algorithm is developed by benefiting from the movement capability of nodes. The simulations worked in this thesis show that the proposed algorithm has better maximum communication range than the existing ones in the literature. In addition, reliable data transfer is also obtained. 
The new proposed algorithm also has better retransmission and delay performance.

\subsection{Thesis Organization}

The outline of the thesis is organized as follows.

In Chapter 2, some background information and definitions are given related to underwater acoustic channel and its characteristics. The challenges of underwater acoustic communication networks are explained in detail. In addition, MAC Layer,

Transport Layer and Routing Protocols and Topology Control algorithms are investigated and evaluated for underwater communication networks.

Chapter 3 theoretically analyzes the communication networks. The related theories and sonar equations are also mentioned. The communication range formula is obtained for underwater communication networks.

Chapter 4 presents proposed algorithm for underwater communication networks. The details of the algorithm are given in this chapter.

Chapter 5 begins with the review of simulation tools. Then, our simulation setup and works are given in detail. In the rest of the chapter, the simulation scenarios and their results are discussed.

In Chapter 6, the thesis is concluded with discussions of the study. Moreover, some ideas for related future works are evaluated. 


\section{CHAPTER 2}

\section{LITERATURE REVIEW}

In order to provide foundation for the proposed work in this thesis, basic concepts of underwater acoustic channel and underwater acoustic sensor networks will be provided. Moreover, current literature on MAC, routing and transport layer protocols and topology control algorithms is discussed and reviewed in this chapter. Protocols and algorithms are briefly explained and summarized according to their solution approaches.

\subsection{Underwater Acoustic Channel Characteristics}

Underwater acoustic network has become an important topic with research interests about acoustics and underwater applications. Collection of oceanographic data, seismic monitoring, disaster prevention, tactical surveillance, ocean-bottom pollution monitoring can be considered as underwater applications. To implement these applications, the underwater acoustic (UWA) channel characteristics should be investigated. Understanding of UWA characteristics is highly important to realize these applications. However, channel characteristics of underwater communication are significantly different from the one of terrestrial communication. The UWA channel is characterized as limited bandwidth, effect of multipath and fading, rapid time variation, large Doppler shift, large propagation delay, low data rate, high bit error rate(BER), limited power when compared to terrestrial communication channel. These challenges are explained in [1], [2], [3], and [4]: 
- Bandwidth in underwater communications is very limited since acoustic channel has strong attenuation with increasing frequency. It depends on the transmission distance because of high noise at low frequencies (lower than $1 \mathrm{kHz}$ ) and high absorption at high frequencies (greater than $50 \mathrm{kHz}$ ).

- UWAC is affected by the multipath interference caused by reflections and refractions. It causes frequency-selectivity of the channel. This frequencydependency causes fading underwater.

- As the speed of the sound under water $(1500 \mathrm{~m} / \mathrm{s})$ is 200000 times lower than the speed of light, large propagation delay occurs in underwater acoustics.

- Large propagation delay and the movement of underwater devices such as transducers, ocean surface, etc. lead to Doppler Effect and rapid time variation.

- Limited bandwidth and large propagation delay affect data rate of networks. In underwater communication networks, data rate is relatively low compared to RF communications.

- Because of underwater environment, acoustic signals sometimes cannot reach the receiver. This results in temporary loss of connectivity (shadow zones) in underwater networks. Connectivity loss, Doppler Effect, multipath, time variation and low SNR increase bit error rate (BER).

- Batteries limit power and cannot be recharged. Thus, there is limited power in underwater.

- Underwater devices and sensors are more prone to failures of fouling and corrosion.

\subsection{Underwater Acoustic Sensor Networks}

Sensor nodes are deployed under water to activate underwater applications such as oceanographic data collection, disaster prevention and tactical surveillance 
applications. Unmanned Underwater Vehicles (UUV) and Autonomous Underwater Vehicles (AUV) with underwater sensors are also made in use of exploration of natural resources under water, gathering scientific data, etc. To realize these applications, the underwater acoustic network is needed to be used. Sensor nodes and vehicles should coordinate their operations by exchanging configuration, location information and they should relay data to sink.

Underwater acoustic communication networking is the technology that presents the opportunity of enabling these applications. Underwater Acoustic Sensor Networks (UASN) consists of underwater sensors and vehicles that are deployed to execute their missions. UASN must be able to organize these sensors and vehicles and adapt to the characteristics of underwater medium. Moreover, UASN is based on the acoustic wireless technology [76].

Sensor nodes under water can be connected as centralized, distributed, and multihop topologies for underwater acoustic sensor networks [69]. Centralized networks use a central station to manage data flow between other sensor nodes. These networks are connected to a backbone at the central station. They are more suitable for deep-water acoustic sensor networks. A major disadvantage of centralized topologies is existence of a single point failure. If the master node fails, then the entire network will not operate. On the other hand, the other two topologies are classified as peer-to-peer networks. They provide point-to-point links between all nodes of networks.

Since UASN uses the underwater communication channel, the same challenges of the underwater acoustic channel that are mentioned previous part are valid for UASN. It is significantly different from terrestrial sensor networks. The unique features of the UASN are given in [40].

- The UASN has low bandwidth and high latency as the acoustic channel is used as a communication method. The propagation speed of the acoustic sound in water is much lower than the one in the terrestrial network. 
According to [77], any underwater acoustic communication system cannot exceed $40 \mathrm{~km} *$ kbps as the maximum attainable Range * Rate product.

- The UASN is highly dynamic network. The UASN consists of sensor nodes and they have low or medium mobility due to water activities.

- The UASN is more prone to errors compared with terrestrial sensor networks. Underwater environment causes path loss, noise, multi-path, Doppler spread and fading and it affects underwater sensor networks negatively. Therefore, acoustic communication links in the UASN are considerably error-prone because of the underwater environment.

- UASNs are generally 3-dimensional networks. Sensor nodes and vehicles are deployed in 3-dimensional area. This is different from the 2-dimensional deployment of most land-based sensor networks.

These characteristics of UASNs bring about many challenges for every level of protocol stacks.

\subsection{Medium Access Control (MAC) Protocols}

Medium access control or multiple access control (MAC) is a network layer between the data link layer and the medium. MAC layer provides addressing and channel access control mechanisms that help network nodes to communicate within a multiple access network. For underwater networks, MAC protocols organize the access to the underwater acoustic communication channel. The main objective of MAC protocols is to avoid collisions. Moreover, MAC protocols make contributions to the network performance, such as increasing throughput, decreasing latency, and increasing energy efficiency [5]. MAC protocols can be divided into two schemes:

i) contention-free schemes

ii) contention-based schemes 


\subsubsection{Contention-Free Schemes}

Contention-free schemes are Frequency-Division Multiple Access (FDMA)-based, Time-Division Multiple Access (TDMA)-based and Code-Division Multiple Access (CDMA)-based.

The Classical FDMA protocol assigns different frequency bands to different nodes. This provides nodes to communicate at the same time without interfering in other nodes. However, in the underwater communication, since bandwidth is limited and multipath propagation occurs, FDMA is stated to be unsuitable for the underwater acoustic communication. Orthogonal Frequency-Division Multiple Access (OFDMA) is another FDMA-based protocol. In OFDMA, medium access assigns subsets of subcarriers to individual network users. OFDMA gives an opportunity of low data rate transmission to multiple users [6]. OFDMA may be suitable for underwater networks when Orthogonal Frequency-Division Multiplexing (OFDM) technique is used in physical layer.

TDMA approach divides the time into small slots and provides time guards to limit collisions for same time slots. It is very difficult to realize precise synchronization because of the underwater environment. Furthermore, TDMA is not efficient in the underwater communication as the UWA channel has high propagation delay and delay variation [7]. Due to these factors TDMA protocols are not suitable for underwater communications. Static TDMA(s-TDMA) [6] and Dynamic TDMA (dTDMA) [8] are TDMA-based protocols.

CDMA protocols allow multiple users to share frequency band with a special coding scheme by assigning different code to each users. CDMA performance is over FDMA performance by robustness of the frequency selectivity and over TDMA performance by accessing nodes simultaneously. According to [2], CDMA protocol is a good choice for the underwater communication. Moreover, Frequency Hoping Spread Spectrum (FHSS) CDMA and Direct Sequence Spread Spectrum (DSSS) CDMA are proposed for underwater applications in [1]. UW-MAC [9] and Interleave-Division Multiple Access (IDMA) [10] are CDMA-based protocols 
developed for underwater communications. On the other hand, when one of nodes is stronger than another node, the interference from the stronger one affects demodulation of the weaker one. This, called as a near-far problem, affects CDMAbased protocols negatively. Thus, although CDMA-based protocols can be considered as a promising for underwater communications, they are not suitable due to the near-far problem.

\subsubsection{Contention-Based Schemes}

Contention-based schemes can be categorized as Request-to-Send (RTS)-Clear-toSend (CTS) exchange based, Carrier Sense Multiple Access (CSMA)-based, reservation-based and ALOHA-based protocols [11].

ALOHA protocol has a simple method of transmitting the data in communication. In ALOHA, when the data is wanted to send, transmitter immediately sends the data without channel sensing or retransmission [11]. ALOHA may be a good choice for networks that has small packet transmission time and collision unlikely. SlottedALOHA [12] protocol is an ALOHA-based protocol. Slotted-ALOHA provides random access without the reservation. The time is divided into slots, like TDMA. The data is transmitted at the beginning of each time slot.

Multiple Access with Collision Avoidance (MACA) [13], RTS-CTS exchange based protocol, has a handshaking algorithm to reserve channel. Firstly, transmitter sends RTS to reserve channel for data transmission. RTS carries information about length of the data packet to be transmitted. When RTS is received, receiver replies CTS packet also including packet length if the channel is not used and not reserved. Then, sender begins to transmit the data when CTS is received. MACA protocol solves the hidden terminal problem, but it does not solve the exposed terminal problem. MACA-Wireless (MACAW) [14] protocol, extended version of MACA, is an RTSCTS exchange based protocol with acknowledgment on the data link layer. MACAW helps channels of low reliable network to increase throughput. On the other hand, MACA for Underwater (MACA-U) [15], also an extension of MACA 
protocol, is proposed for multi-hop underwater networks. In MACA-U, nodes have states and transition rules. MACA-U has better throughput than classical MACA approach in underwater networks. Another RTS-CTS exchange based protocol is Propagation-Delay-Tolerant Collision Avoidance Protocol (PCAP) proposed for underwater networks [16]. PCAP tries to solve the propagation delay problem in underwater. The receiver delays CTS for two times of propagation delay in underwater after RTS is received in PCAP. The requirement of synchronization can be the main disadvantage of PCAP. Adaptive Propagation-Delay-Tolerant Collision Avoidance Protocol (APCAP) [17], the RTS-CTS exchange protocol, is an improved version of the PCAP. In APCAP, the time between reception of RTS and sending of CTS is adaptive with an algorithm to improve efficiency and throughput of networks. Moreover, Distance Aware Collision Avoidance Protocol (DACAP) [18] is another RTS-CTS exchange protocol proposed for underwater networks due to high propagation delay. DACAP minimizes the handshaking time by improving tolerance of receiver node for interference when the nodes are closer than the maximal transmission power. Spatially Fair MAC Protocol (SF-MAC) [19] is proposed protocol for underwater acoustic sensor networks. SF-MAC is also an RTS-CTS exchange based protocol. SF-MAC determines transmission sequentially at the receiver side after receiver node waits for RTS from all nodes. It can avoid collisions and guarantee the fairness of the transmission for underwater communications. Furthermore, another proposed RTS-CTS exchange based protocol is Dual Busy Tone Multiple Access (DBTMA) [20]. DBTMA provides parallel handshaking by dividing channel into two sub-channels: the data channel and the control channel. It deals with the exposed node problem for underwater networks.

Floor Acquisition Multiple Access (FAMA) [21] is a CSMA-based protocol. When two nodes want to reserve the same channel simultaneously, collision may occur. Collisions, due to this problem, are prevented by increasing RTS-CTS packets. Although FAMA solves the collision problem, high packet delays and waste of energy occur due to multiple RTS-CTS transmission in underwater networks. In addition, an extended version of FAMA is developed as a Slotted-FAMA [22]. 
Slotted FAMA provides time slots for RTS-CTS exchange instead of excessively long control packets. The length of slot is the addition of maximum propagation delay and CTS transmission time. It also guarantees the collision-free transmission. Ordered-CSMA [23] is another CSMA-based protocol developed for underwater acoustic networks. It is basically a combination of CSMA and Round-Robin Scheduling. Instead, nodes wait for a time period of maximum propagation delay; the sender immediately begins to transmit the data after transmission of last node in the order. In addition, Propagation Delay-Aware Opportunistic Transmission Schedules (DOTS) [24], a CSMA-based protocol, is developed for underwater networks. This approach uses local information passively obtained to increase concurrent transmissions. It also reduces collisions. However, clock synchronization is needed for DOTS and it is a problem for underwater communications. Lastly, Queue-Length-Based CSMA (Q-CSMA) [25] is a CSMA-based protocol. Q-CSMA divides time slots into control and data slots. Q-CSMA provides collision-free data transmission schedule while allowing collisions in control slots. Thus, Q-CSMA is appropriate for static networks.

UWAN-MAC, reservation-based approach, is an energy-efficient MAC protocol for underwater acoustic wireless sensor networks [26]. UWAN-MAC protocol focuses on energy efficiency. Nodes keep sleep mode until the specific timeout period is up after it is synched from transmitter node. The protocol needs synchronization through periodic data exchange. Another reservation-based MAC protocol is Tone Lohi (T-Lohi) [27]. Tones are used in T-Lohi during contention periods to reserve the channel. Contender detection comes from listening the channel after it sends reservation time. Moreover, Reservation-based MAC(R-MAC) protocol [28] is an energy efficient MAC protocol for underwater sensor networks. In R-MAC, each node periodically states listen and sleep modes. All nodes have same listen and sleep mode durations. Centralized scheduling and synchronization are not needed since each node determines its own schedule randomly. R-MAC increases energy efficiency. Lastly, Receiver-Initiated Reservation-Based Protocol (RIPT) [29] is developed for underwater acoustic networks. In RIPT, every node needs to know the 
inter-node propagation delay between itself and its neighbors. The receiver reserves the channel with packet train. This is not a suitable protocol for dynamic network topologies.

\subsection{Data Link Layer Protocols}

Data link layer protocols are needed to make reliable data transport in networks. ARQ (Automatic Repeat Request) is a basic mechanism in the data link layer to deliver information through the higher layers keeping overhead, retransmissions, and discarded information as low as possible.

ARQ is an error-control method for the data transmission that uses acknowledgements and timeouts to achieve reliable data transmission over an unreliable service. If the sender does not receive an acknowledgment before the timeout, it usually re-transmits the frame/packet until the sender receives an acknowledgment or exceeds a predefined number of re-transmissions.

Forward error correction (FEC) is an important contributor to ensure low bit error rates. FEC reduces the error rates in the physical layer, such that data link layer protocols have a better chance of being successful.

For the underwater acoustic channel, physical link has a poor quality due to multipath and Doppler effects. This results in high bit error rate (BER). Thus, data link layer protocols, such as ARQ mechanisms, are required to achieve reliable data transfer in underwater networks.

Classical ARQ techniques are Stop-and-Wait ARQ, Go-Back-N ARQ and Selective Repeat ARQ. Firstly, in Stop-and-Wait ARQ, source node transmits data packets and when destination node receives them it replies ACK. Source node waits for ACK before sending next data packets. When the underwater acoustic channel has high round-trip time, Stop-and-Wait ARQ is inefficient for underwater networks. Moreover, the source node sends a great deal of enumerated data frames continuously in one transmission for Go-Back-N ARQ mechanism. When the errors 
occur, the destination node sends back the number of the first data frame received in error. However, Go-Back-N ARQ consumes too much energy and time. Due to limited energy and time varying environment in underwater acoustic channel, GoBack-N is not suitable for underwater networks. Lastly, in Selective Repeat ARQ, the source node transmits a great deal of enumerated data frames continuously in one transmission, same as Go-Back-N ARQ. On the other hand, the destination node sends back the sequence number of all data frames received as errors. For the best transmission, the source node only sends these erroneous frames. Although Selective Repeat ARQ has better time and energy performance than Stop-and-Wait ARQ and Go-Back-N ARQ, it is not so efficient for underwater networks due to low throughput [5].

Modified Stop-and-Wait ARQ [30] is proposed for underwater communication networks as a transport layer protocol. In this mechanism, classical Stop-and-Wait ARQ approach is improved with transmitting data packets in groups and receiving ACKs selectively to increase throughput. According to network properties, such as range, data rate, error probability, optimal packet size is selected. Moreover, Multihop ARQ [31] is also an improved Stop-and-Wait ARQ mechanism. It acknowledged implicitly or explicitly in multi-hop networks. Multi-hop ARQ provides better latency and energy efficiency performance.

Hybrid ARQ approach is basically combination of ARQ and FEC. The simplest form using FEC at the physical layer and ARQ at the link layer is called as Type-1 Hybrid ARQ. On the other hand, using incremental redundancy check ARQ is called as Type-2 Hybrid ARQ. It relies on FEC code to provide different amount of redundancy. Type-1 Hybrid ARQ and Type-2 Hybrid ARQ were compared in [32]. According to the comparison, Type-2 Hybrid ARQ outperforms Type-1 Hybrid ARQ. Furthermore, Underwater Hybrid ARQ (UW-HARQ) [33] is developed for underwater acoustic networks. UW-HARQ combines Random Binary Linear Coding as a FEC coding and ARQ. An adaptive packet error ratio (PER) estimation scheme is proposed for minimization of retransmissions. In addition, ARQ uses NACK packets to inform transmitter number of packets sent out in a transmission, and it 
uses ACK packets to indicate success of recovered data packets. Lastly, Segmented Data Reliable Transport (SDRT) protocol [34] is another hybrid ARQ approach for underwater networks. It uses erasure codes, called SVT codes that transfer encoded packets block by block and hop by hop. The advantages of SDRT are few numbers of retransmissions, good channel utilization, and simple protocol management.

Cooperative ARQ [35] protocol is proposed for underwater acoustic networks. Reliable alternative paths for the source to the destination connection are achieved by using cooperative nodes in the cooperative ARQ. It is based on knowledge of inter-node distances. When an error occurs, the cooperative node selects a node for retransmission according to closest-one-first rule. This selection continues until the data is transferred to the next cooperative node. This ARQ scheme improves throughput of network.

\subsection{Routing Protocols}

Routing is said to be crucial part of any wireless network. Routing protocols can provide better energy efficiency, better throughput of the network, and more reliable data transmission. Routing protocols play an important role for the data transmission in underwater acoustic networks. Due to high propagation delay, multi-hop topologies are more feasible than single-hop ones. Spreading loss factor and absorption losses cause attenuation in underwater communication. Because of high attenuation, high power at the transmitter node is needed in order to provide sufficient Signal-to-Noise Ratio.

Routing protocols can be divided into three sub-groups: Proactive Routing, Reactive Routing, and Geographical Routing.

\subsubsection{Proactive Routing}

Proactive routing protocols find the path between the source and the destination and they exchange the network topology information periodically. Since the routing 
paths are established in advance and are provided, proactive routing protocols have an advantage to achieve good end-to-end delay. However, they are not good at high load and high mobility conditions. Optimized Link State Routing (OLSR) [36] and Destination-Sequence Distance-Vector Routing (DSDV) [37] are mostly used proactive routing protocols. Moreover, in proactive routing, to establish a route for the first time large overhead signaling is provoked. Thus, proactive routing protocols are not suitable for underwater communication networks when limited bandwidth conditions are considered.

\subsubsection{Reactive Routing}

Reactive routing protocols work finding path only when the data is transmitted. In reactive routing, low traffic and routing overhead are controlled. The advantage of them is to work well for mobile networks. Thus, they are more suitable for networks having dynamic environments. Ad-hoc On Demand Distance Vector (AODV) [38] and Dynamic Source Routing (DSR) [39] are known as common reactive routing protocols. On the other hand, latency is high in reactive routing protocols and a source is required to initiate control packets that establish routing paths. Because of high latency in routing protocols and high propagation delay of the underwater environment, reactive routing is not appropriate for underwater networks.

\subsubsection{Geographical Routing}

In geographical routing protocols, the data packet is progressively advanced from the source to the destination. Every network station has at least one neighbor to relay data for geographical routing. The geographical routing protocols have many advantages. It is virtually stateless and limited signaling is required. However, local disconnections may occur in networks and the geographical routing protocols are based on knowledge of nodes' positions. 
Vector-Based Forwarding (VBF) [40] is a location-based routing approach. In VBF algorithm, nodes will transmit data packets if it is close to the data forwarding vector from the source to the destination. It is assumed that nodes know their positions and they can measure the distance and the angle to the forwarder for this algorithm. The forwarding vector is actually said to be a virtual pipe from the source to the destination and only the network nodes in this pipe can participate in the data transmission. Furthermore, Hop-by-Hop Vector-Based Forwarding (HH-VBF) is a developed-VBF approach. This protocol also uses the virtual pipe. The main difference is that the forwarder node is decided by per hop forwarding path. HHVBF provides better forwarding path for the networks having limited number of nodes.

Depth-Based Routing (DBR) [42] protocol is proposed to provide scalable and effective routing without the localization process. DBR only needs depth sensors, not full localization information, for routing in underwater acoustic networks. DBR is an efficient routing protocol for dynamic dense networks. In DBR protocol, the data is forwarded to the sinks in water surface. Data packets include depth information of its forwarder. This information is updated in every node participating in active data transfer. When the data packet arrives at the node, the node calculates its distance to the water surface and it forwards the data packet if its depth is smaller than the depth information of previous node in data packet. There are two queues, namely priority queue and packet history buffer, to handle data packet retransmissions for energy consumptions in DBR.

Hop-by-Hop Dynamic Addressing Protocol (H2-DAB) [43] is a flooding-based routing protocol. Each node has an ID, called as a hop ID. This hop ID is determined by the help of "hello message" transmitted from the sink node. The packet transfer occurs according to hop ID of a node. The proposed transmission for $\mathrm{H} 2-\mathrm{DAB}$ is as follows. The sender node transmits an "inquiry packet" message. Nodes respond this message with "inquiry reply" message including their hop ID if they are in communication range. Then, the sender node transmits the data packet through the node that has a smaller hop ID than the others. 
Focused Beam Routing (FBR) [44] is proposed for avoiding redundant flooding of broadcast queries. In FBR, the source node must be aware of its positions and also the position of destination. Moreover, every node is aware of its positions. The routes are established during the packet transmission.

Reliable and Energy Balanced Routing (REBAR) protocol [45] is another locationbased approach. REBAR uses geographical information of nodes. Each node has a specific ID and it is assigned to the fixed range. According to the REBAR algorithm, source node sends the data including the information about distance $\mathrm{d}$ between source and sink node towards the vector $\mathrm{V}$ through the sink. When intermediate node receives the packet, it calculates distance di to the sink. If di-d is less than the threshold distance pre-defined by the algorithm, the node will participate in the data transmission to the sink.

Sector-Based Routing with Destination Location (SBR-DLP) [46] is a locationbased routing protocol. SBR-DLP performs the flooding by dividing the communication area into the different sector areas. SBR-DLP algorithm assumes that every node in the network knows its position and the planned movement of destination node. Data forwarding is done by the hop-by-hop for this algorithm.

In Directional Flooding-Based Routing (DFR) [47], transmission of data packets is done in a limited flooding zone. This zone is selected according to the angle between source-to-receiver vector and receiver-to-destination vector. DFR assumes that every node knows the geographical position of all other nodes. In addition, each node is also capable of measuring links quality.

Location Aware Source Routing (LASR) [48] is proposed for high-latency of acoustic communication links by using two techniques, namely location awareness and link quality metrics. LASR assumes that network has symmetric links and small number of nodes. The packet transmission is performed according to the link quality metrics and location of neighbor nodes.

Multipath Power-control Transmission (MPT) [49] is a location-based approach for energy optimization. In MPT algorithm, firstly the available multiple routing paths 
are obtained from source node by sending request message to intermediate nodes and receiving reply messages from them. Then, the source node determines the optimal paths according to path lengths.

Moreover, it calculates optimal overall energy consumption for these routing paths. This calculation is made by the help of collected information during establishment of routing path phase. Therefore, the packets are delivered based on the established paths and calculated energy consumptions.

Minimum Cost Clustering Protocol (MCCP) [50] is a cluster-based protocol. The cluster costs are computed by the required energy by the cluster nodes to reach the cluster-head node, the cluster-head node's position according to sink node and residual energy of cluster nodes and cluster-head node. The packet transmission occurs according to computed cluster costs.

Distributed Underwater Clustering Scheme (DUCS) [51] is also a cluster-based approach. DUCS algorithm does not need the location information of nodes. It is based on distributed scheme approach. Firstly, the algorithm forms clusters by selecting cluster-head node and cluster nodes. The cluster-head node is determined by the residual energy. Then, cluster-head node specifies its cluster nodes. The data packet transmission occurs based on propagation delays of cluster-head node and its cluster members.

In underwater networks, acoustic signals can give better precision than radio signals. Locations of nodes in underwater can be determined by using beacons placed in known locations. Therefore, geographical routing protocols are promising for underwater communication networks. 


\subsection{Topology Control}

\subsubsection{Definition of Topology Control}

The efficient use of energy resources and the reliable data transfer for ad-hoc and sensor networks are fundamental tasks of the network designers. Reducing energy consumption and increasing quality of service are important issues for communication networks. To enhance network quality and energy consumption, network topology can be controlled. Topology Control is the art of coordinating nodes' decisions in order to generate a network with desired properties while reducing energy consumption and/or increasing network capacity. The topology control does not impose any constraints on the nature of the mechanism. Thus, topology control can be centralized or distributed techniques [52].

Topology control is classified into two categories as homogeneous critical transmission range (CTR) and nonhomogeneous topology control. On one hand, in homogeneous CTR, all nodes in the network use the same transmitting range and topology control problem becomes determining the minimum value of the transmission range such that desired network property is satisfied. On the other hand, nodes are allowed to use different transmission ranges in nonhomogeneous networks. The nonhomogeneous case is categorized into three categories, depending on the type of information used for computation of topology. Firstly, in location based approaches, node positions are used. Secondly, in direction-based techniques, nodes do not know their positions, but they can estimate the direction of their neighbors. Thirdly, in neighbor-based approaches, nodes are assumed to have access to minimum information regarding their neighbors [52].

According to [53], topology control techniques can be systematically classified into two categories: network coverage and network connectivity. Firstly, network coverage technique is related to how well the target field is monitored by the sensor network. It aims to reach a reliable sensing area while consuming less power. 
Secondly, network connectivity is related to efficient sensor connectivity topology with controlling and managing power.

The topology control protocol have an interaction with both routing layer and MAC layer protocol. Yet, the place of the topology control mechanism in protocol stack is not clear in the literature and the integration of topology control techniques in the protocol stack is seen one of the open research areas [52]. Hence, it can be between MAC layer and routing layer or close to one of them.

\subsubsection{Topology Control Algorithms}

Most of topology control algorithms aim to solve the energy problem of networks in radio communication. Network devices consume a great amount of energy by sending or receiving data. Thus, the transmission power control is important for the energy problem. Energy efficient topology control algorithm for both fixed and variable power [54] is developed to maximize the lifetime of the network by using minimum spanning tree construction. Furthermore, three energy-aware geographical routing and topology control forwarding schemes are proposed to improve network lifetime by considering residual energy of neighbor nodes in deciding next hop [55].

To increase network capacity and prolong network lifetime, distributed topology control algorithm is developed and proposed for a network of heterogeneous devices [56]. Topology control is done by adjusting power transmission per node for sensor networks in this algorithm. [57] is also proposed as a distributed topology control algorithm based on the directional information for heterogeneous networks. According to this algorithm, the transmitter node increases transmission power until finding a neighbor node in every direction. Another topology control algorithm, MobileGrid [58], targets to achieve best possible network capacity by contention index via dynamically adjusting the transmission range on each of nodes in networks.

Minimum Routing Cost Connected Dominating Set (MOC-CDS) based topology control algorithm is improved and proposed for topology control problem with 
routing cost constraints in wireless networks [59]. MOC-CDS provides efficient routing and network performance.

In [60], a cooperative, distributed and lightweight topology control approach is proposed to adapt transmission power of sensors in order to match local connectivity for underwater applications.

Interference Prediction-based Topology Control (IPBTC) [61] is proposed for threedimensional wireless sensor network. This algorithm makes use of the interference factor control to predict the interference probability of different links and determines the link selection criterion. IPBTC can provide the network topology with low interference and energy balance characteristics.

Since underwater environment have a challenging characteristics for communication, topology control techniques in underwater applications are considerably different from those in terrestrial networks. Some topology control algorithms are developed for underwater acoustic sensor networks.

For underwater sensor networks, QoS-Based Topology Control Algorithm is developed [62]. It is a depth-based strategy adjustment algorithm for better network performance. Moreover, in [63], another similar topology control approach, distributed radius determination algorithm, is presented. It is also a depth-based approach for mobility-based topology control problem to improve network performance.

Topology control strategy-based on complex network theory (TCSCN) [64] is a topology control algorithm for underwater sensor networks. Two-cluster structure is constructed in this algorithm and cluster-heads are used to ensure connectivity and coverage for $3 \mathrm{D}$ hierarchical topologies. In addition, TCSCN optimizes energy consumption and propagation delay.

Opportunistic Localization by Topology Control (OLTC) [65] is proposed against unlocalization of sensor nodes for underwater sensor networks. OLTC uses a gametheoretic model based on Single-Leader-Multi-Follower Stackelberg game for 
topology control of both localized and unlocalized nodes. It increases localization coverage performance of UWSN. Moreover, OLTC is an energy-efficient algorithm. Topology control algorithms for underwater applications in the literature mostly aims to improve energy efficiency. Moreover, small networks are not considered in these algorithms. Although TCSCN is developed to increase network coverage and connectivity, small networks are not considered in this approach. 


\section{CHAPTER 3}

\section{SONAR EQUATIONS AND ANALYSIS}

In this chapter, sonar equations are investigated and presented. Detection threshold concept is also given. Maximum transmission range for underwater communication is derived from sonar equations. How to obtain the maximum transmission range is explained in detail.

\subsection{Sound Propagation}

The sound propagates several different paths in sea. The particular travelled paths for sound are dependent of the sound speed characteristics. When the sound speed characteristics in sea are investigated, it is seen that the depth (z), the salinity (s) and the temperature (T) of water affects the sound speed [66]. Therefore the sound speed is formulated by;

$$
\begin{aligned}
c=1448.96+ & 4.591 * T-0.05204 * T^{2}+0.0002374 * T^{3}+1.340 \\
& *(s-35)+0.0163 * z+1.675 * 10^{-7} * z^{2}-0.01025 \\
& * T *(s-35)-7.139 * 10^{-13} * T * z^{3}
\end{aligned}
$$

Equation 3.1 is valid for $0 \leq T \leq 30^{\circ}, 30 \leq s \leq 40$ and $0 \leq z \leq 8000$.

The many specialties of underwater acoustic sound make an impact on design characteristics of sonar systems and behavior of sonar equipment. These effects can be logically grouped as a sonar parameters and sonar equations. Sonar parameters are mostly related to underwater sound and medium characteristics. Moreover, sonar equations reflect the relationship between the medium, the source and the target 
equipment for underwater sound applications. There are two concepts for sonar systems, namely passive sonar and active sonar. If the sonar equipment is acting as both sound source and receiver, it is called active sonar system. In passive sonar case, on the other hand, sonar equipment's either produce the signals or receive them.

One node transmits the signals to the other node in underwater acoustic communication. Therefore, the underwater acoustic communication channel can be considered as a passive sonar. The passive sonar equation can be seen in equation

$$
S L-T L=N L-D I+D T
$$

SL: the source level that represents the signal power transmitted from the sound source

TL: the transmission loss that is the absorbed loss from the underwater medium

NL: the noise level of underwater medium

DI: the receiving directivity index

DT: the detection threshold that is the minimum power level of receiver to detect the signal

\subsection{Source Level}

The source level (SL) in sonar equations is defined as the amount of sound power transmitted from the sound source. It is the intensity of a plane wave of rms pressure $1 \mathrm{uPa}$, referred to a point 1 yard from the acoustic center of the projector in the direction of the target. Since the directional projector points in the direction of the target, the reference point for source level lies along the axis of the projector's beam pattern [67]. 


$$
S L=171.5+10 \log P_{\text {elec }}+10 \log E+D I_{T}
$$

$D I_{T}$, called transmitting directivity index, is the power level difference between the power level produced by the sound source and the power level that would be produced by the non-directional source.

$P_{\text {elec }}$ is the electrical input power produced from the source. On the other hand, $E$ is the electro-acoustic power conversion efficiency of the transducer. Sonar system efficiencies mostly vary between 0.2 and 0.7 .

$$
E=\frac{P_{\text {acoustic }}}{P_{\text {elec }}}
$$

$P_{\text {acoustic }}$ is the total acoustic input power radiated from the transducer.

\subsection{Transmission Loss}

Sound is highly propagated in the sea water depending on the physical and the chemical properties of water. The sound propagation causes the power reduction from the source level (SL) generated by the sound source through the underwater medium. This reduction is called as a transmission loss (TL). The definition of the transmission loss (TL) is the weakening of the sound between 1 yard from the source and a point at a distance in the water. The transmission loss depends on the frequency of a signal and the path distance. In general, the TL can be given by

$$
T L(l, f)=\left(l / l_{r}\right)^{k} * \alpha(f)^{l-l_{r}}
$$

where $l$ is the transmission path distance, taken in reference distance $l_{r}, f$ is the signal frequency, and $k$ is the spreading loss coefficient. $\alpha(f)$ is the absorption loss coefficient.

The transmission loss can be divided into two physical factors: Absorption Loss and Spreading Loss. 


\subsubsection{Absorption Loss}

Absorption loss is a loss dependent to the characteristics of medium. It covers the conversion process of acoustic energy into heat. Furthermore, it represents true loss of acoustic energy to the medium in which propagation is taking place [67]. Absorption loss can be approximately calculated or measured with fixed parameters (e.g. temperature, salinity, $\mathrm{pH}$ values) of the underwater medium.

According to the Thorp's formula, the absorption loss coefficient under water is given in Equation 3.6.

$$
\alpha(f)=\frac{0,11 * f^{2}}{1+f^{2}}+\frac{44 * f^{2}}{4100+f^{2}}+3 * 10^{-4} * f^{2}+3,3 * 10^{-3}
$$

$\alpha(f)$ is the absorption loss coefficient in $\mathrm{dB} / \mathrm{km}$ and $\mathrm{f}$ is the operating frequency in $\mathrm{kHz}$.

$$
\text { Absorption Loss }=\alpha(f) * R * 10^{-3}
$$

Absorption Loss is in $\mathrm{dB}, \mathrm{R}$ is the distance between source and receiver in $\mathrm{m}$.

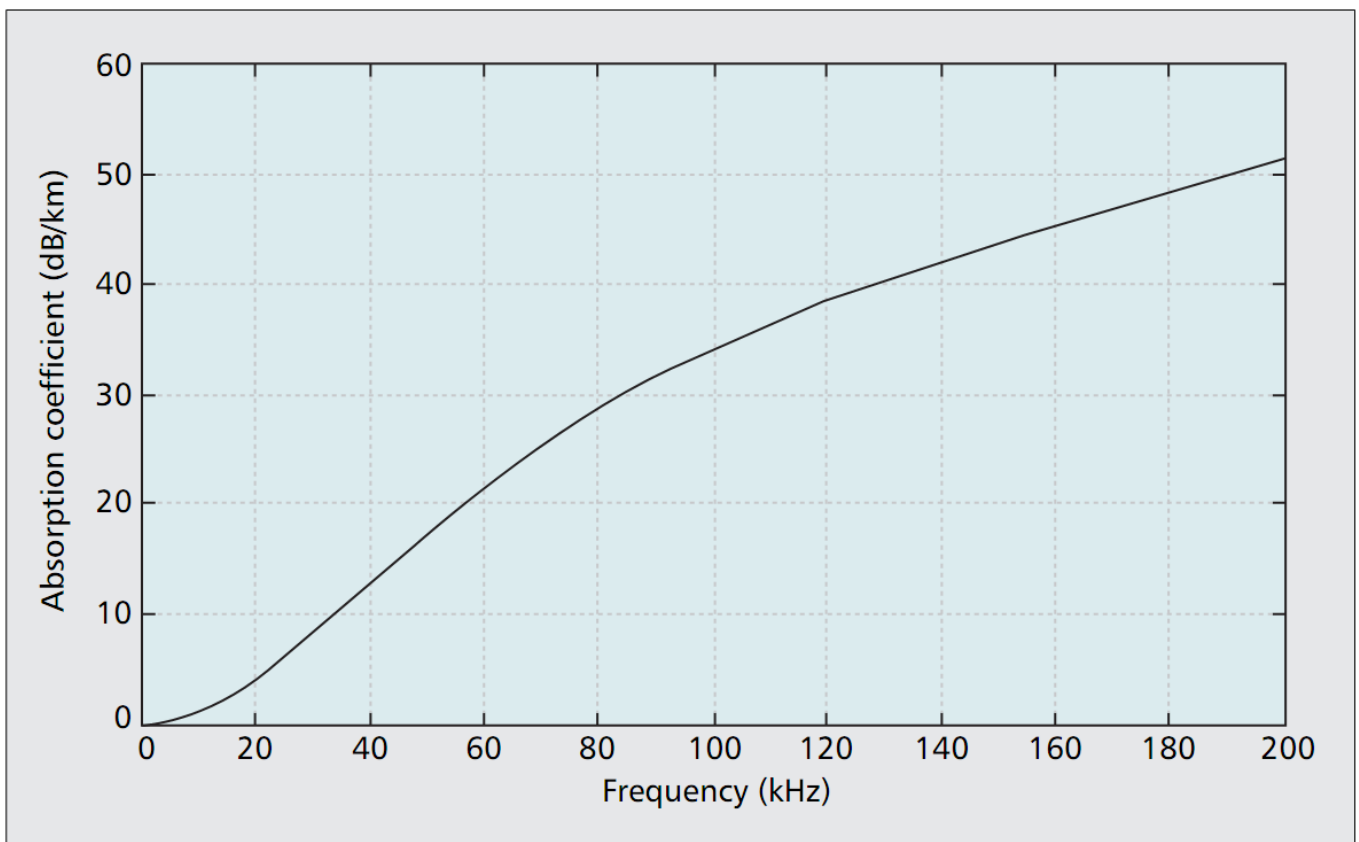

Figure 3.1: Absorption loss coefficient characteristics, $10 \log \alpha(f)$ [68] 
When equation 3.6 and Figure 3.1 are considered, the absorption loss coefficient is dramatically increasing with frequency. Thus, the transmission loss increases with increasing frequency. The operational frequency should be selected low frequencies so that the long transmission distance is achieved for underwater communication channel.

\subsubsection{Spreading Loss}

Spreading loss, geometrical factor that affects the transmission loss, is the weakening of a sound signal as it is spreading outward from the sound source. Therefore, while the sound signal is travelling from the source through the destination, it losses power due to the spreading. The spreading loss is changing with the water depth as the acoustic signals differently propagate within the different depths in water. Acoustic signal sound propagates within a cylinder bounded by the surface and the sea floor in shallow water whereas it spreads spherically in deep water. As a result, the absorption loss is different for deep water and shallow water.

For shallow water, the spreading loss is cylindrical spreading and the equation 3.8 is valid for the distance $\mathrm{R}$ from the sound source.

$$
\text { Spreading Loss }=10 \log R
$$

On the other hand, the spreading loss is spherical spreading for deep water and the equation 3.9 is valid for the distance $\mathrm{R}$ from the sound source.

$$
\text { Spreading Loss }=20 \log R
$$

Consequently, the transmission loss becomes as an equation 3.10 for deep water.

$$
T L=\alpha(f) * R * 10^{-3}+20 \log R
$$




\subsection{Ambient Noise Level}

Ambient noise level is the total noise background of the sea. It can be observed with a non-directional hydrophone. The ambient noise level is the intensity of the noise background, in decibel. It is referred to the intensity of a plane wave having an rms pressure of $1 \mathrm{uPa}$. Although ambient noise levels are measured for different frequency bands, they are always normalized to a $1-\mathrm{Hz}$ band. Thus, they are called ambient-noise spectrum levels.

The sources of ambient noise are basically turbulences in sea, ships, waves of water, and temperature of water.

\subsubsection{Turbulence Noise}

The form of irregular random water currents of large or small scale, called turbulence, creates the noise background in several ways. It is called turbulence noise. Shakes of hydrophone, pressure changes by the water current are the major factors that compose the turbulence noise. Turbulence noise $N_{t}(f)$ is formulated in the equation 3.11 .

$$
10 \log N_{t}(f)=17-30 \log f
$$

\subsubsection{Shipping Noise}

Ship traffic in sea creates the noise background, called shipping noise. The shipping noise is dominant source for ambient noise level for especially in $100 \mathrm{~Hz}$ [67]. It is described by the formula in 3.12 .

$$
10 \log N_{s}(f)=40+20 *(s-0.5)+26 \log f-60 \log (f+0.03)
$$

where $s$ is the shipping activity factor whose value is varying between 0 and 1 . The value of 0 and 1 represents low and high shipping activity, respectively. 


\subsubsection{Wave Noise}

One of the ambient noise sources is the wave-generation action of the wind on the surface of the sea. It is called wave noise caused by the wind force. The noise level is related with the wind speed over the hydrophone. The wind force generates the waves having different wavelengths that travel the surface with different speeds depending on their wavelengths as it is always turbulent near the sea surface. Long waves can travel with a speed greater than sound speed in the sea and they will radiate pressure waves into the sea. This is the characteristics of wave noise. Therefore, the wave noise level depends on the speed of wind and frequency.

$$
10 \log N_{w}(f)=50+7,5 * w^{0,5}+20 \log f-40 \log (f+0.4)
$$

where $\mathrm{w}$ is the wind speed, in $\mathrm{m} / \mathrm{s}$.

\subsubsection{Thermal Noise}

Thermal noise is another source of the ambient noise. Temperature of the molecules of the sea places the limit to the hydrophone sensitivity for especially high frequencies. The thermal noise level can be calculated in equation 3.14.

$$
10 \log N_{t h}(f)=-15+20 \log f
$$

\subsubsection{Deep Water Spectra}

When the overall ambient noise is considered for deep water, an example of the spectrum in Figure 3.2 is observed at one deep-sea location [67]. The ambient noise spectrum has different behavior for different frequency bands under different conditions. For different frequency bands, some noise sources are dominant whereas some are passive. 


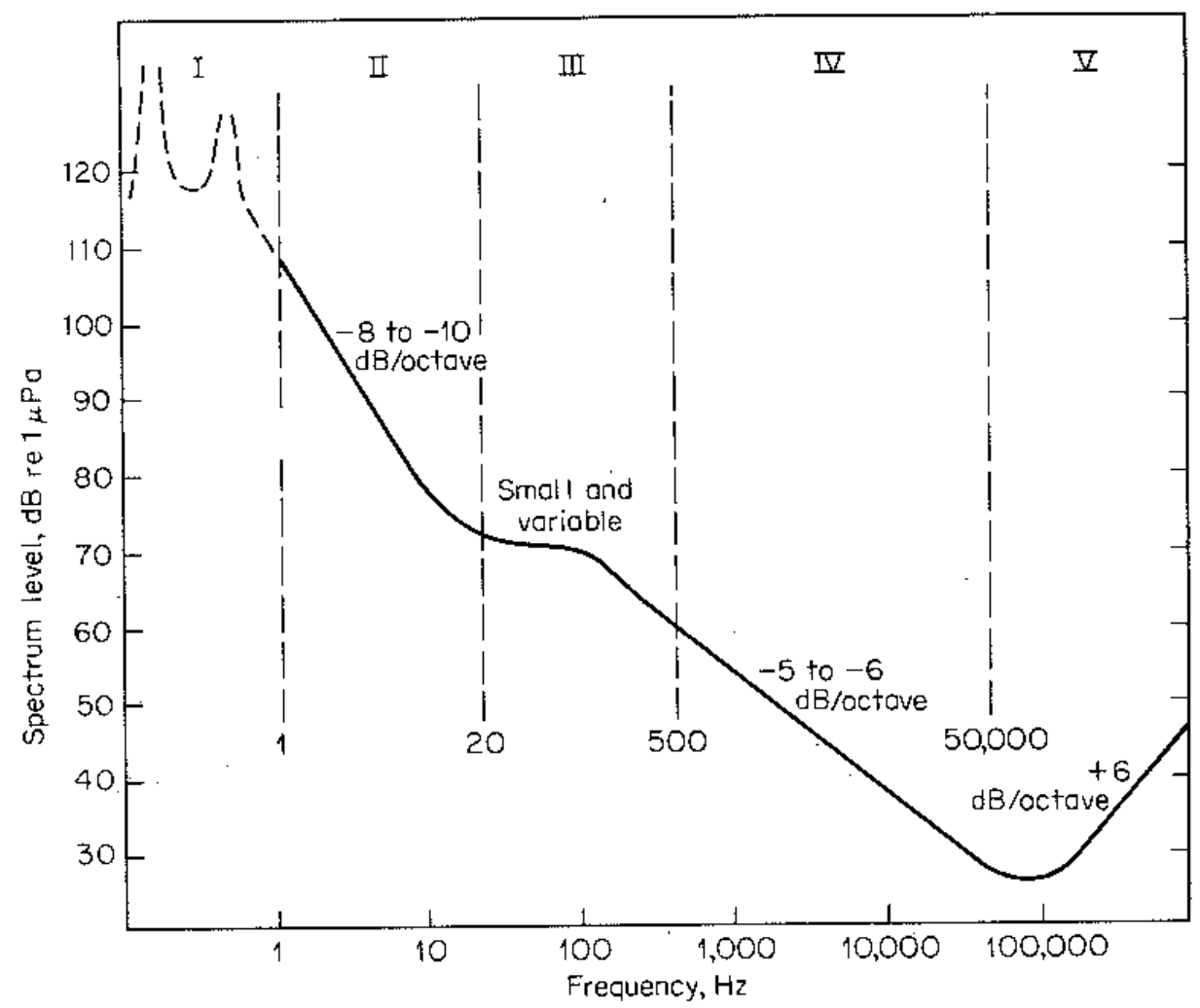

Figure 3.2: The sample spectrum of the deep-sea noise showing different frequency bands with different spectral slopes [67]

The sample deep-sea ambient noise spectrum is seen in Figure 3.2 for different frequency bands with different spectral slopes. The noise level, in $\mathrm{dB}$, can be calculated for specific frequency with the help of the spectrum graph in this figure.

\subsection{Directivity Index}

Sonar systems may have hydrophones assembled in arrays. When this type of hydrophones is used, there is an improvement on the signal-to-noise $\operatorname{ratio}(\mathrm{S} / \mathrm{N})$. The improvement of the $\mathrm{S} / \mathrm{N}$ is known as an array gain. The array gain is defined as in equation 3.15 . 


$$
\text { Array Gain }=10 \log \frac{(S / N)_{\text {array }}}{(S / N)_{\text {element }}}
$$

However, it is difficult to compute the array gain since it relies on knowing the coherences of a signal and noise across the dimensions of the array. On the other hand, for the important case that the signal is coherent and a plane wave, and the noise is incoherent and isotropic, the array gain can be reduced as easily visualized quantity, called as the directivity index(DI) [69].

$$
D I=10 \log \frac{\text { peak intensity of radiated pattern }}{\text { average intesity of radiated pattern }}
$$

The hydrophone array type is important for directivity index. It can be line array, planar array, square array, circular array and cylindrical array. Therefore, directivity index formula can be specified and derived for different hydrophone array types.

\subsection{Detection Threshold}

Sonar systems can hear the sea in any time. However, the obtained signal may be the sound signal or the noise background. Firstly, sonar systems should detect the presence of the sound signals in spite of the noise background. After that, they can perform other system functions. Some pre-assigned level for the signal-to-noise ratio is required in order to make a decision and detect the sound signal. At this preassigned level for signal-to-noise ratio is called the detection threshold. It refers to input terminals of the receiver, display and observer combination. According to sonar equations, the detection threshold is a term that satisfies the equality for these equations when the sound signal is just being detected.

Detection threshold (DT) is defined as the ratio of the signal power in the receiver bandwidth to the noise power in $1-\mathrm{Hz}$ band, measured at the receiver, required for the detection at some pre-assigned level of the detection decision. 


$$
D T=10 \log \frac{S}{N}
$$

where $\mathrm{S}$ is the signal power in the receiver and $\mathrm{N}$ is the noise power in a $1-\mathrm{Hz}$ frequency band at the receiver.

In fact, when a signal is detected at the receiver input terminals, there are two possibilities for decision: signal is present and signal is absent. On the other hand, for the other possibility of signal, there are again two possibilities that can occur. Therefore, when the all detection situations are considered, the four possibilities occur. The possibility of signal detection table is given in Figure 3.3 by detecting the signal at the receiver input terminal.

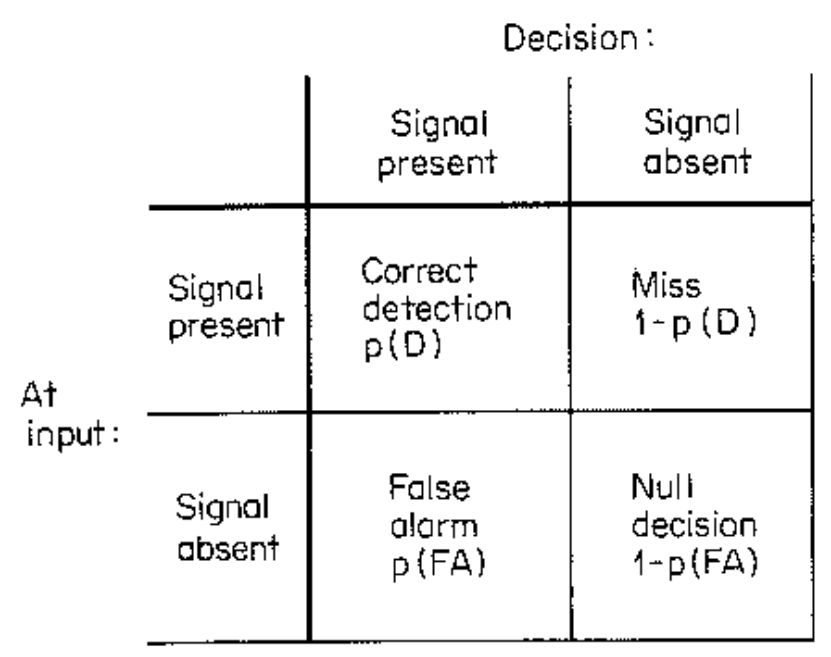

Figure 3.3: Decision matrix [67]

Decision matrix and probabilities is shown in figure 3.3. According to the matrix, The probability of presence of the signal that if the signal is correctly decided, "signal present" is made is called detection probability, p(D). On the other hand, the probability of absence of the signal that if the decision is incorrect, "signal present" is made is called the false alarm probability, $\mathrm{p}(\mathrm{FA})$. 


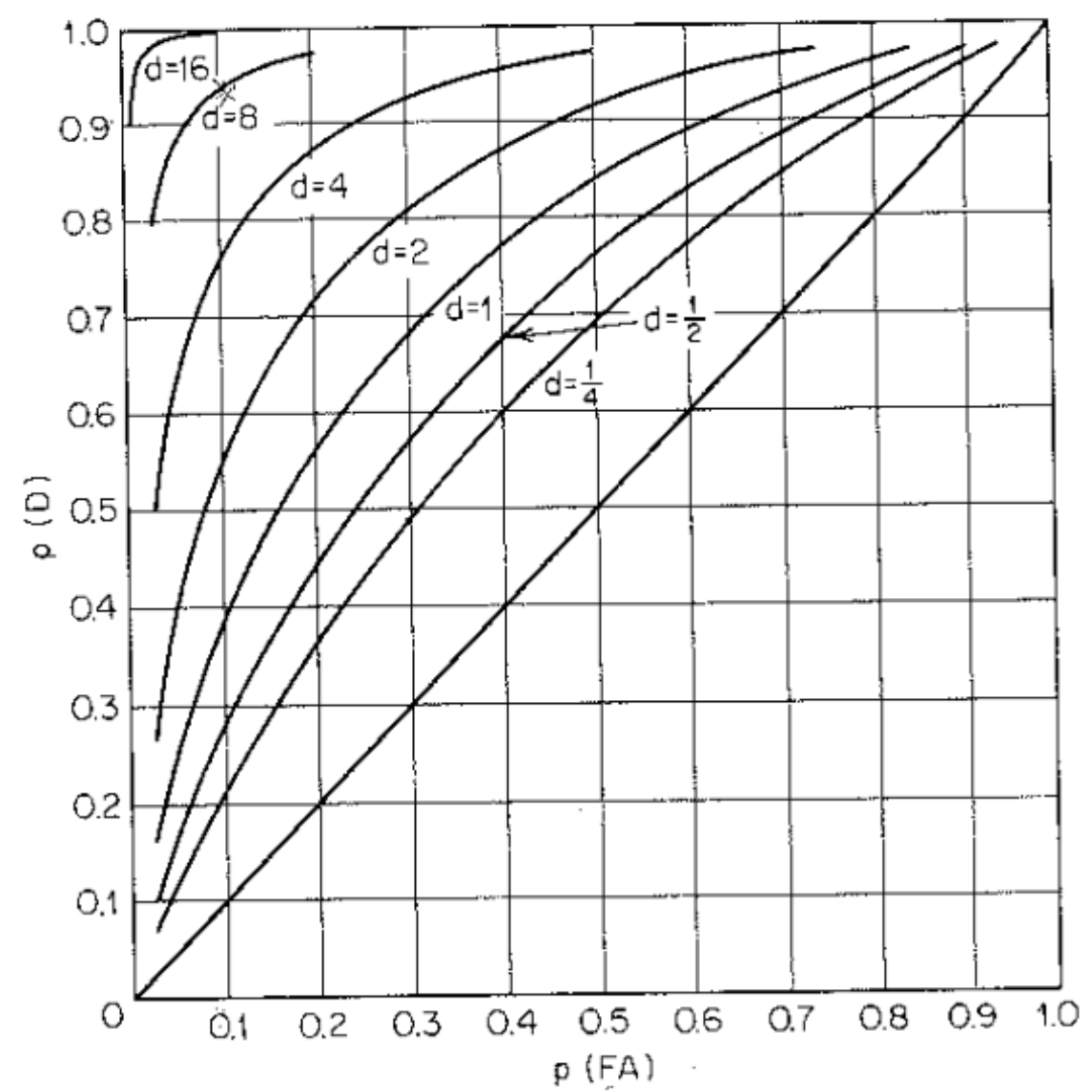

Figure 3.4: Example ROC curve of detection probability $p(D)$ against false-alarm probability $\mathrm{p}(\mathrm{FA})[61]$

When the output signal-to-noise ratio is considered as a fixed value, different threshold settings bring about different pair of values of the two decision probabilities. The correct detection probability and the false-alarm probability can be plotted as a curve according to each other for different threshold settings. This curve graph is called as receiver-operating-characteristic (ROC) curves. An example of ROC curve graph can be seen in Figure 3.4.

The parameter $\mathrm{d}$ is the detection index that is equivalent to the signal-plus-noise to the noise ratio of the envelope of the receiver output at the terminals for established threshold settings [67]. The detection index, $d$, is calculated by the formula in 3.18. 


$$
d=\frac{\left(M_{S+N}-M_{N}\right)^{2}}{\sigma^{2}}
$$

where $M_{S+N}$ is the mean signal-plus-noise amplitude, $M_{N}$ is the mean noise amplitude and $\sigma$ is the variance of probability-density functions.

\subsection{Transmission Range Calculation}

The transmission range is limited for underwater acoustic channel due to the challenges such as high propagation delay, high absorption loss. The maximum distance that two nodes can communicate successfully can be determined by the help of sonar equation in 3.2.

At the sound source side, transducer is assumed as non-directional characteristics. Thus, the transmitter directivity index becomes zero.

The deep-water conditions are assumed and ambient noise level can be calculated by the help of Figure 3.2.

At the receiver side, hydrophone is assumed single, not array type. Then, the receiver directivity index can be ignored.

The detection threshold can be estimated according to the ROC curve graph in Figure 3.4.

When all above conditions and assumptions are considered, the maximum transmission distance $R_{\max }$ can be found by the help of the equation 3.19 .

$$
\begin{gathered}
\alpha(f) * R_{\max } * 10^{-3}+20 \log R_{\max }=171.5+10 \log \left(P_{\text {elec }} * E\right)- \\
N L-D T
\end{gathered}
$$

$P_{\text {elec }}, E$ are related to the transducer at transmitter side and they are known. $N L$, $D T$ are related to sea conditions and assumptions and they are also known. The operating frequency is decided. Therefore, the only unknown variable is $R_{\max }$ and it can be calculated. 


\section{CHAPTER 4}

\section{TOPOLOGY CONTROL VECTOR BASED FORWARDING PROTOCOL}

Communication range and connectivity loss problems for underwater acoustic networks are investigated and the topology of underwater acoustic networks is modeled. A novel approach, namely Topology Control Vector Based Forwarding Protocol (TC-VBF), is proposed for these problems. The descriptions, the parameters and the packet transfer algorithm of the protocol are also given in this chapter.

\subsection{Topology Model for Underwater Acoustic Sensor Networks}

In UASNs, when two nodes want to communicate, the distance between them should be lower than the maximum transmission distance of nodes for a successful data exchange. On the other hand, routing algorithms can increase the communication distance by relaying the data with the help of other nodes in the network.

Underwater acoustic sensor networks can be classified as small, medium or large networks according to number of nodes they have. All of three network types may suffer from the loss of connectivity due to the underwater environment. It is a big challenge to handle connectivity losses in the network.

It can be assumed that large UASNs, deployed highly dense with sensor nodes, can be considered as a combination of many clusters. Assume that while nodes inside these clusters are able to communicate with each other, the node which is closest to the neighbor cluster could not communicate with the neighbor cluster because of the 
connectivity loss. The reasons of this loss are the node's mobility and the underwater medium. Therefore, the entire network can be modeled as separated clusters and the network loses all connectivity. One of these network models can be seen in Figure 4.1. The network in the figure becomes two separate clusters because of the connectivity loss. The data exchange between these clusters is not possible in this situation since the distance $d$ between two closest nodes in different clusters is greater than the maximum transmission distance.

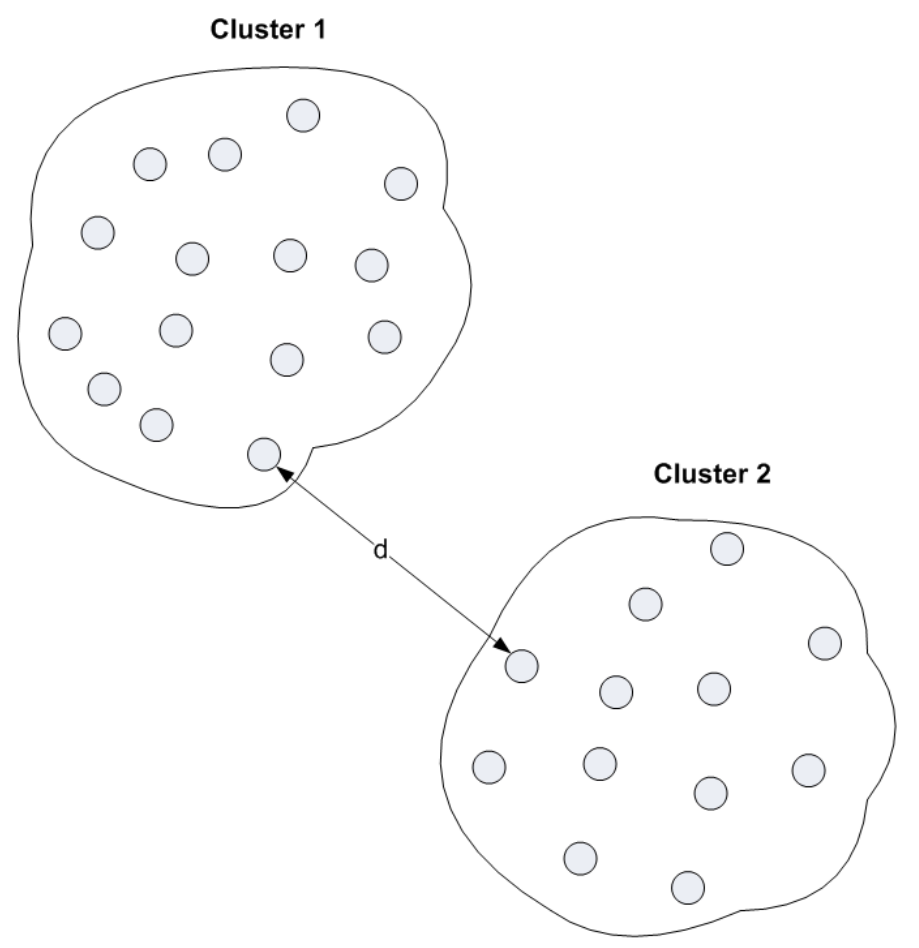

Figure 4.1: The connectivity loss model for large UASN

The connectivity loss problem for large networks can be reduced to the same problem for small or medium networks. According to above figure, all nodes can have the communication ability within the cluster and they could not communicate with the nodes inside the other cluster. Only a small number of nodes in networks can be focused and the topology model is reduced to the model in Figure 4.2. It is assumed that the red-colored node in this figure is the sender node and the node 
outside the cluster is the receiver node. According to this assumption, it becomes the same problem of small networks.

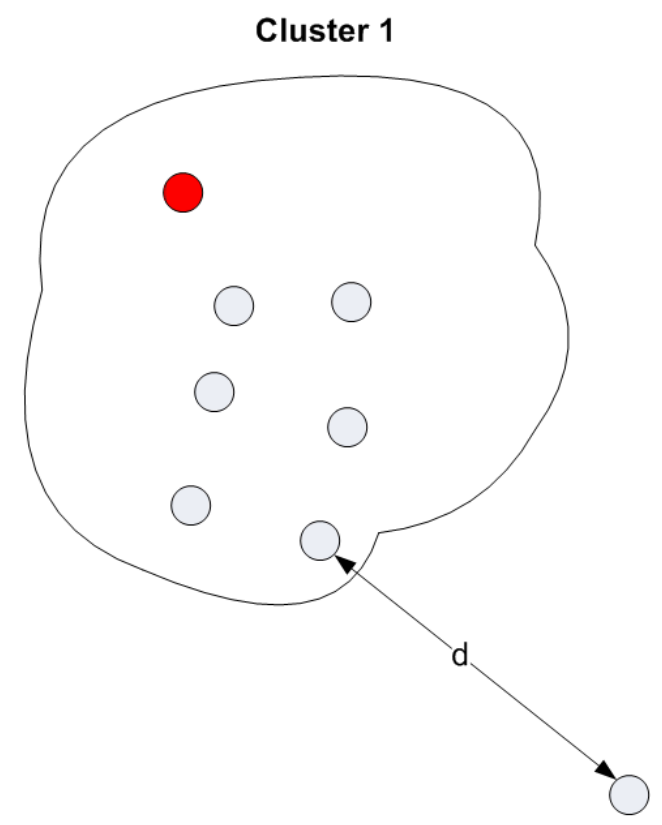

Figure 4.2: Generalized connectivity loss model for UASNs

The sender node, a master node of the network, is assumed to know position information of all nodes in the network. In this situation, it is assumed that the distance between the sender and the receiver node is lower than four times of the maximum transmission distance. This distance can be divided into four equal parts and midpoints are marked with cross sign, shown in Figure 4.3. According to this figure, two nodes are said to be close to two of these crossed points. If the bluecolored node should have been close to the other crossed point, there would not be any connectivity loss. Hence, if the blue-colored node is moved to the third crossed point by controlling topology, the connectivity loss can be eliminated. After that, packets are transmitted with the help of nodes inside the data vector shown in Figure 4.4. The data path for packets can be also seen in this figure. Therefore, connectivity losses for all underwater acoustic sensor networks can be handled by controlling topology. 


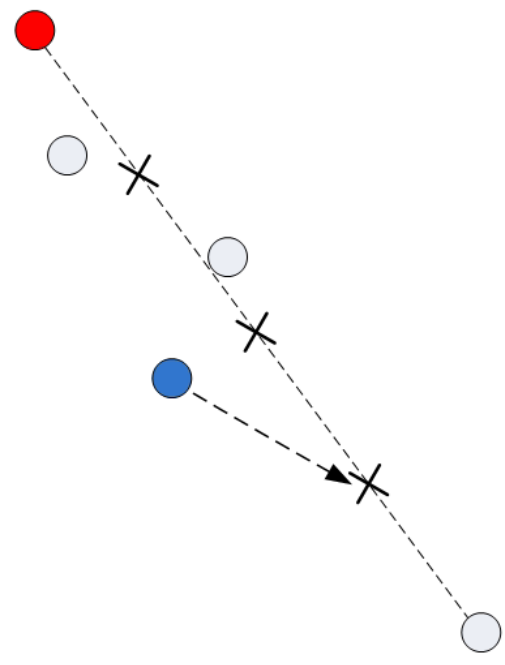

Figure 4.3: The topology control mechanism against the loss of connectivity

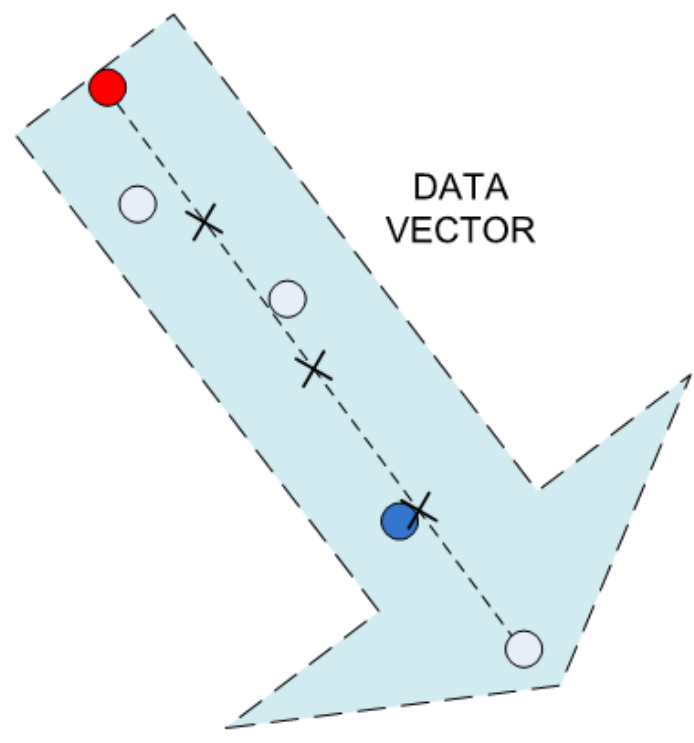

Figure 4.4: The data vector and the data path for packet transfer

\subsection{VBF Overview}

In UASN, node mobility causes to rapidly change the network topology due to the strong multipath. Vector-Based Forwarding Protocol (VBF) is developed to 
overcome this problem. Therefore it provides energy efficient, robust and scalable routing. VBF is a location-based routing approach. In the VBF, there is a vector with pre-defined radius from the source node to the destination node. Then, the message will be forwarded by the nodes close to the vector. This routing process involves a small fraction of nodes in the network. In addition, the VBF reduces the energy consumption of the network by discarding low benefit packets. For the VBF algorithm, the forwarded message carries the information about location of the sender (SP), the target (TP) and the forwarder (FP). The forwarding path is determined by the routing vector from the source to the destination. The neighbor nodes are the decider for the $\mathrm{VBF}$ algorithm. It calculates the distance to the forwarding vector and compares to the predefined threshold distance. If the calculated distance is less than the threshold, then the neighbor node forwards the packet with adding its position. Otherwise, the packet is discarded. In other words, if the neighbor node is in the virtual pipe with a predefined radius, it is actively participated in data transfer. Moreover, there is a RANGE field in the data packet to solve node mobility.

Data packet in the VBF also includes a RADIUS field. The RADIUS field is used for holding the radius information of forwarding virtual pipe.

For routing initiation, there are two query types for packets: sink initiated query and source initiated query. Sink initiated query uses sink-based coordinate system and it is location-dependent. The sink sends INTEREST message carrying the coordinates of sink and target. For source initiated query, on the other hand, source firstly send DATA_READY message with setting source originated coordinate system. These INTEREST and DATA_READY packets are forwarded by relaying in the virtual pipe.

VBF has a self-adaptation algorithm to reduce energy consumption. This algorithm determines the most desirable nodes for relays according to the desirableness factor. It is measured by the node capability of relay operation. 


\subsection{Topology Control Vector Based Forwarding Protocol}

\subsubsection{Introduction}

The networks with small number of nodes have a short communication range. Moreover, due to high absorption loss and high propagation delay of the underwater acoustic channel, the transmission range is negatively affected. When these conditions are considered for small networks, the communication range for underwater channel becomes limited. The VBF algorithm can increase the communication range but not always to the desired level.

In order to increase the communication range and enhance the communication coverage, a novel approach for underwater acoustic networks is proposed. It is called Topology Control Vector Based Forwarding Protocol (TC-VBF). The TCVBF is developed from the VBF algorithm with the topology control approach. It is also location-based, scalable, robust and reliable protocol.

The TC-VBF approach is developed for underwater acoustic networks that have small number of nodes. It is master-node controlled algorithm. However, this algorithm is not aimed to provide better transmission delay for underwater acoustic networks. The transmission delay is expected to be high for TC-VBF algorithm as well.

\subsubsection{Protocol Description}

The TC-VBF algorithm is proposed for master-controlled networks having small number of nodes. All data packets are mainly sent from the master node. All of the other nodes, slave nodes, are the potential sink node.

The properties of the TC-VBF are as follows:

- Master node knows the position information of all nodes. Slave nodes only know their own positions and the position of the master-node. 
- Each node has the unique ID. Master node knows all node's ID with their position.

- The threshold distance, pre-defined critical transmission distance, is known by all nodes in the network.

- Slave nodes have the moving ability whereas master node does not.

- The packet includes the data with the master node position, the sink node position, the forwarder node position, and the sink node ID.

- The initiation process is needed for determining the forwarding path of data packets.

\subsubsection{Threshold Distance (TD)}

The critical transmission distance for node plays a crucial role for the networks. The node's electrical, acoustic and physical properties have an impact on this critical distance. For instance, the more transmission power the transducer of transmitter node can send, the greater is the transmission distance. The receiver node having more sensitive hydrophone can hear the acoustic signal for longer distance. However, the improvement of these properties of nodes is difficult and expensive. Additionally, the improvement is also limited.

The critical transmission range is derived from the sonar equations and given in Chapter 3.

$$
\begin{gathered}
\alpha(f) * R_{\text {ctd }} * 10^{-3}+20 \log R_{\text {ctd }}=171.5+10 \log \left(P_{\text {elec }} * E\right)- \\
N L-D T
\end{gathered}
$$

The threshold distance (TD) is a pre-determined value for networks. TD is the critical transmission distance at which master node and sink node can communicate with desired lowest SNR. It can be calculated from the equation 4.1. Then, the TD is used for the algorithm of the TC-VBF. 
According to the TC-VBF approach, the threshold distance has an important role. When the master node wants to send a data packet, it firstly calculates the distance between its position and the sink node's position. After then, it decides how it sends the data to the sink according to the calculated distance with respect to the TD.

\subsubsection{Packet Transfer Algorithm}

The packet transfer is initiated by the master node in the TC-VBF algorithm. To begin with, the master node calculates the distance to the sink. Then, it divides the calculated distance to the TD. The result of this division is called k-parameter of the algorithm. Another key parameter for the TC-VBF is parameter N. N is also a predefined value for this algorithm. $\mathrm{N}$ specifies a limit for the maximum communication range to be obtained by the help of the TC-VBF.

The packet transfer algorithm is as follows. If k-parameter is less than 1, the distance is less than the TD and the master node directly sends the packet to the sink. On the other hand, if $\mathrm{k}$ is between 1 and $\mathrm{N}$, the master node tries to find the path for the data transfer. The master node firstly divides the sink distance into equal distances and determines mid-points. Then, it finds the nodes closest to the mid-points. After that, it checks the node's position to the next hop mid-point and the previous hop midpoint. If both the distance between the closest node and the next mid-point and the distance between the closest node and the previous mid-point is less than the TD, no movement is required. However, if it is not, this node is acting and moving to the closest mid-point. This node is called an actor node. The actor node goes to the related mid-point of the path to the sink node. When it goes this location, it replies the acknowledgment message to the master node. This iteration is done for all midpoints by the master node. All required movements are done; the data path becomes ready through the sink. Now, threshold distance conditions are satisfied by the help of the actor node's action. Then, the master node sends the data packet with the classical VBF algorithm. After the packet transmission is done successfully, the actor nodes go back to original positions. They continue their own work. Otherwise, 
if $\mathrm{k}$-parameter is greater than $\mathrm{N}$, distance is so far away that the packet transmission cannot be done.

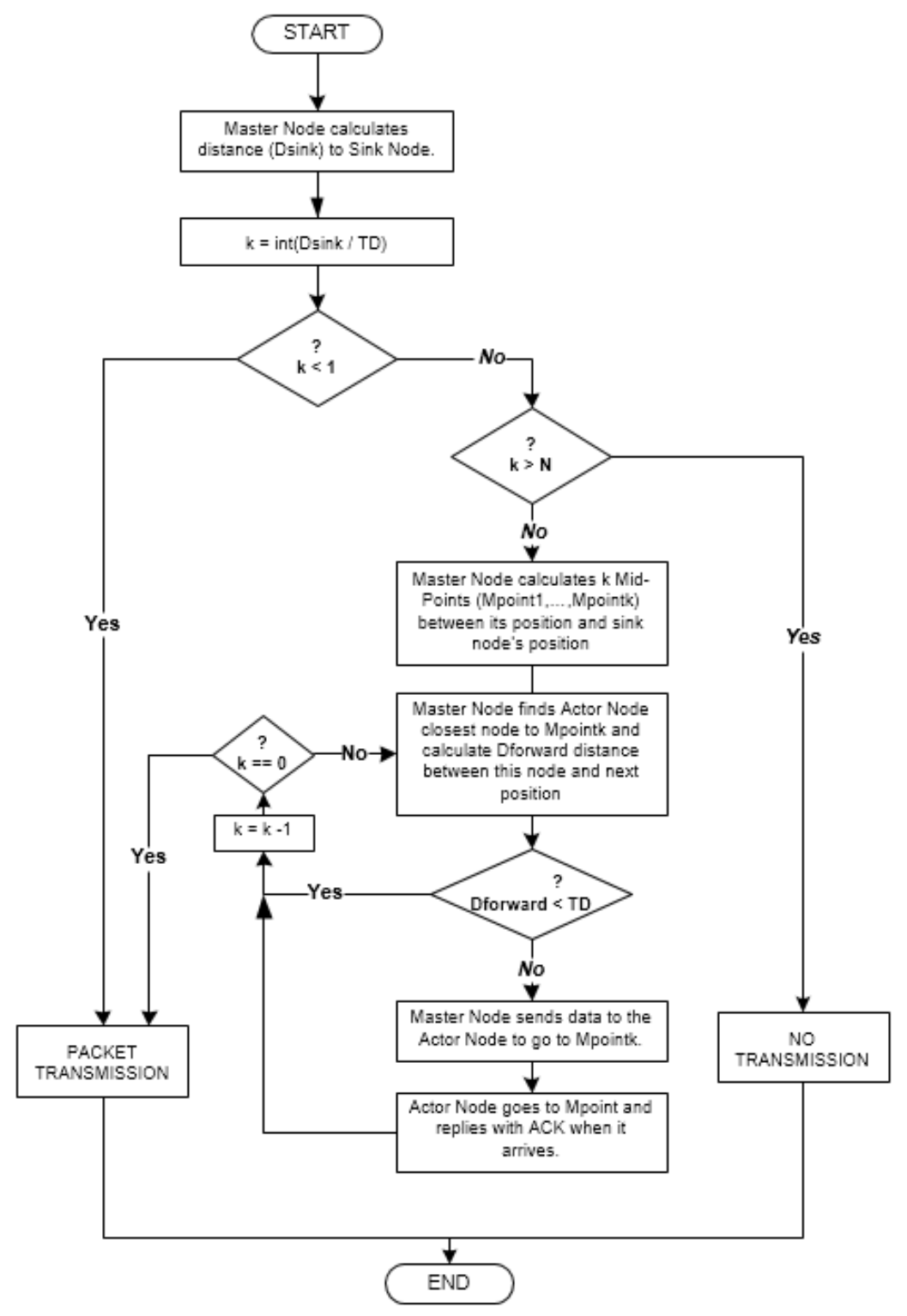

Figure 4.5: Flow chart for TC-VBF approach

The flow chart of the packet transfer algorithm of the TC-VBF is given in Figure 4.4 above. In addition, the master node algorithm is illustrated in detail in Algorithm 1 below. The pseudo code is given in this figure. 


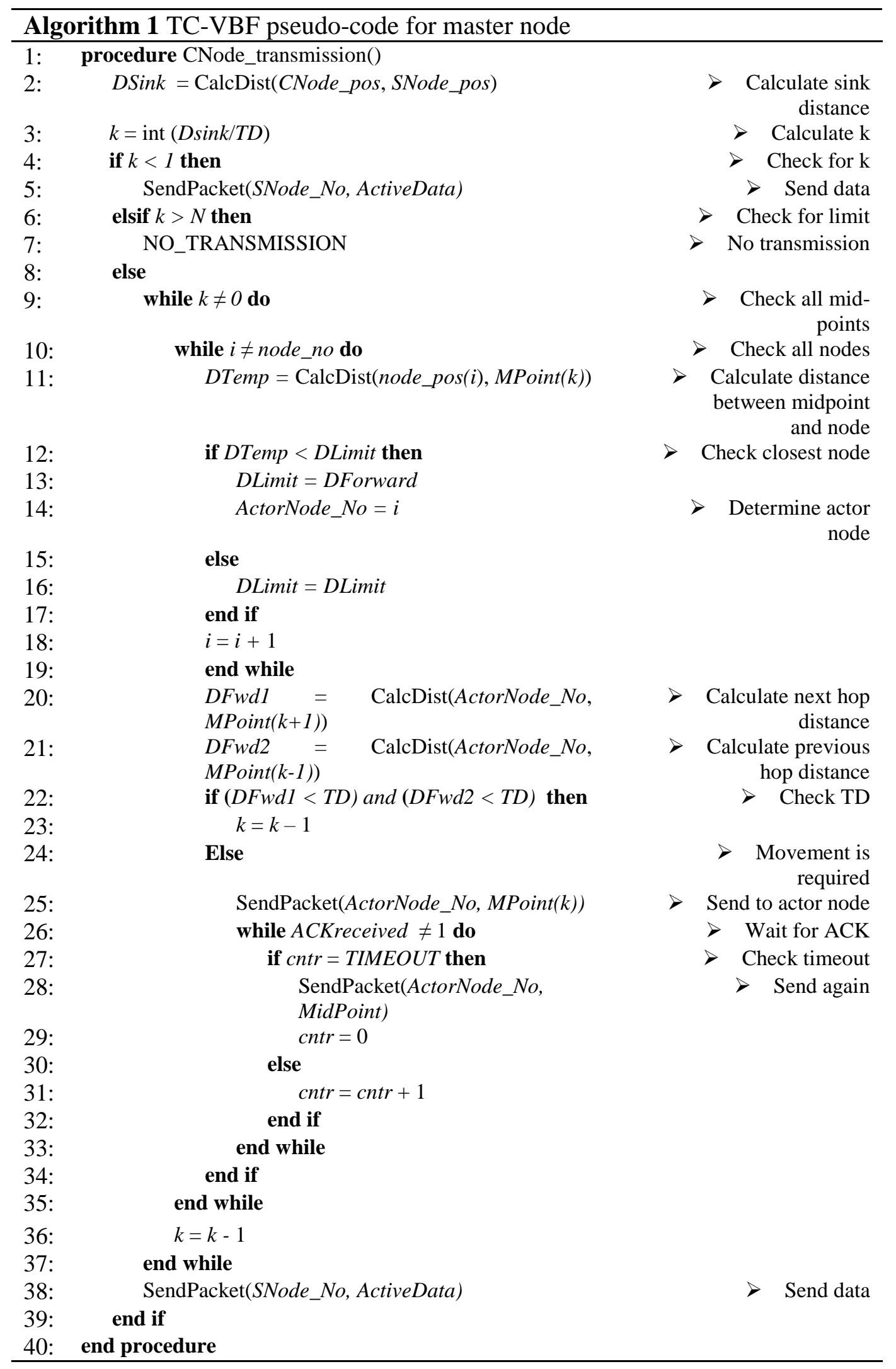


Moreover, the slave node's algorithm is given in Algorithm 2 below.

\begin{tabular}{|c|c|c|c|}
\hline \multicolumn{4}{|c|}{ Algorithm 2 TC-VBF pseudo-code for slave node } \\
\hline 1: & procedure SlaveNode_Reception () & & \\
\hline 2: & if DataReceived $=1$ then & & $>$ Data is received \\
\hline 3: & if ForwardFlag $=1$ then & & $>$ Check forward flag \\
\hline 5: & RelayData() & & $>$ Relay data \\
\hline 6: & elsif ActionFlag $=1$ then & & $>$ Check action flag \\
\hline 7: & ACTION & & $>$ Node is moving \\
\hline 8: & while ActionDone $\neq 1$ do & $>$ & $\begin{array}{r}\text { Wait until movement is } \\
\text { done }\end{array}$ \\
\hline 9: & WAIT & & \\
\hline 10: & end while & & \\
\hline 11: & SendPacket(ACK) & & $>$ Send ACK \\
\hline 12: & while ForwardFlag $\neq 1$ do & & $\begin{array}{r}>\text { Wait forward flag is } \\
\text { active }\end{array}$ \\
\hline 13: & WAIT & & \\
\hline 14: & end while & & \\
\hline 15: & RelayData() & & $>$ Relay data \\
\hline 16: & ACTION_BACK & & Node is moving back \\
\hline 17: & end if & & \\
\hline 18: & end if & & \\
\hline 19: & end procedure & & \\
\hline
\end{tabular}




\section{CHAPTER 5}

\section{SIMULATION BASED PERFORMANCE EVALUATION}

In this chapter, simulation tools are reviewed for underwater applications. MATLAB is selected as a simulation platform. Moreover, all simulation works of the TC-VBF algorithm and results are given in detail. According to simulation results, the TC-VBF approach is said to be promising solution to handle communication range problems of underwater acoustic networks.

\subsection{Simulation Tools Review}

Network protocols are developed to enhance the network performance of underwater acoustic networks. These protocols are investigated by simulations done with the help of tools improved from general network simulators. It can be posited that OPNET, NETSIM, OMNET++, ns2, ns3, JSIM, QUALNET and REAL are appropriate platforms for network simulations. On the other hand, OPNET, ns2 and MATLAB are seen to be used mostly for underwater acoustic networks as simulator tools in the literature review.

MATLAB tool is used to simulate underwater acoustic network applications. In [21], Slotted-Floor Acquisition Multiple Access (Slotted-FAMA) suggested a MAC layer protocol for underwater acoustic network applications is simulated with the help of Simulink on MATLAB. Since MATLAB is a general purpose simulation tool, it is used for the simulation of low level layers [70].

Ns2 (Network Simulater-2), open source software, is a tool that commonly used to simulate network protocols. Ns2 provides TCP (Transmission Control Protocol), routing protocols, multicast protocols for including wired or wireless networks. Ns-2 simulation tool divides channel and physical layer functions into four models: the 
propagation model, the channel model, the physical model and the modulation model. Bellhop, an underwater acoustic ray model, is added to these ns-2 simulation tools for the purpose of using in underwater acoustic network works. World Ocean Simulation System, another ns-2 based simulation tool, is developed by using the Bellhop ray model. ALOHA is compared with Tone-Lohi and Distance-Aware Collision Avoidance Protocol (DACAP) by using the WOSS simulator. Furthermore, other MAC comparison research has been conducted with the help of this simulator [71]. Another Ns-2 based simulation tool is the AquaTools. Any acoustic modem characteristics can be simulated on the AquaTools with appropriate parameters [70]. The Aqua-Sim, similar to the AquaTools, is another Ns-2 based simulation tool. The Aqua-Sim provides to simulate attenuation of the underwater acoustic channel and collision behavior of long delay acoustic networks. In addition, the Aqua-Sim supports three-dimensional networks' propagation and many basic and advanced network protocol models [72].

OPNET is a licensed software simulation tool that is generally used to do simulation works for communication networks. As the OPNET provides the propagation delay stage, the receiver power stage and the background noise stage, real underwater acoustic channel characteristics is obtained by modeling of this channel on OPNET [73]. [74] modifies the standard radio channel to match the characteristics of the shallow water acoustic channel on OPNET. Lastly, [75] tells about the USNET (Underwater Sensor Network Simulation Tools) developed to use simulation of the underwater acoustic network channel.

As a result, OPNET, Ns-2 based simulators, and MATLAB are promising tools for the simulation of underwater acoustic networks.

\subsection{Simulation Setup}

Simulations are done with MATLAB platform. MATLAB is an interactive environment and a high-level language for engineering. Underwater channel and underwater medium are modeled in MATLAB according to sonar equations and 
parameters mentioned in Chapter 3. Furthermore, underwater propagation model is characterized. Hence, all underwater conditions are realized in MATLAB platform with the help of underwater medium properties.

After the underwater channel, the underwater medium, and the underwater propagation delay are modeled, three protocols, VBF, $\mathrm{HH}-\mathrm{VBF}$, and TC-VBF, used in this work are implemented according to their behavior. Implementations of protocols are done with the help of protocol documents in the literature. In both the $\mathrm{VBF}$ and the HH-VBF implementations, the radius parameter is set to $250 \mathrm{~m}$ according to node's physical properties in the network. Furthermore, the transmission distance parameter for the $\mathrm{HH}-\mathrm{VBF}$ protocol is set to $500 \mathrm{~m}$ according to packet error probability graph.

\subsubsection{Point-to-Point Communication Model}

MATLAB underwater channel simulator has a model for packet transfer. The model is used as an underwater point-to-point communication protocol. According to the model, sender node sends a data packet with the receiver node ID information. When the receiver node gets the data packet successfully, it replies an ACK message. If the sender node could not get the ACK, then it retransmits the data packet and this process will continue till the fourth transmission attempt. If the packet is not transferred for the fourth attempt, it is counted as a fail packet.

Furthermore, for the relay operation, when the actor node receives the packet, it buffers for a specified time and then it sends the packet to the next node. Packet transfer occurs according to same process mentioned before. On the other hand, actor node also forwards the ACK message sent from sink node to the previous node in the transmission vector. When the ACK reaches to the master node, the point-topoint communication is completed. 


\subsubsection{End-to-End Delay Model}

Average end-to-end delay graphs in simulations are obtained from the calculations by a delay model of underwater channel simulator. The delay model calculates endto-end delay values according to physical parameters of underwater medium. Furthermore, average end-to-end delay values are averaged for successful packets only.

The delay model works according to point-to-point communication scenario. Firstly packet generation time is calculated from the bit rate. Then, the distance between two nodes is calculated and the propagation delay is obtained for this distance. This calculation is repeated for each packet transfer between two nodes including relays. Speed of sound is assumed $1500 \mathrm{~m} / \mathrm{sec}$. Moreover, ACK message is also considered for the propagation delay. On the other hand, if there is a relay operation, a node buffer time is considered for each relay operation. The node buffer time is assumed 10milliseconds. Lastly, a movement time is obtained for the distance and the node velocity if the node movement occurs for the data transfer. After the delay model calculates all these values, the average end-to-end delay value is eventually obtained by adding these values.

\subsubsection{Simulation Parameters}

Simulations are done in two-dimensional area. As deep water conditions are used, sound spreading is evaluated as a spherical spreading in the simulator.

Signal-to-Noise (SNR) calculation is done according to sonar equations. Both source level and noise level values given above are used. A simulator communication channel is modeled according to the underwater medium and physical parameters, shown in Figure 5.1. 


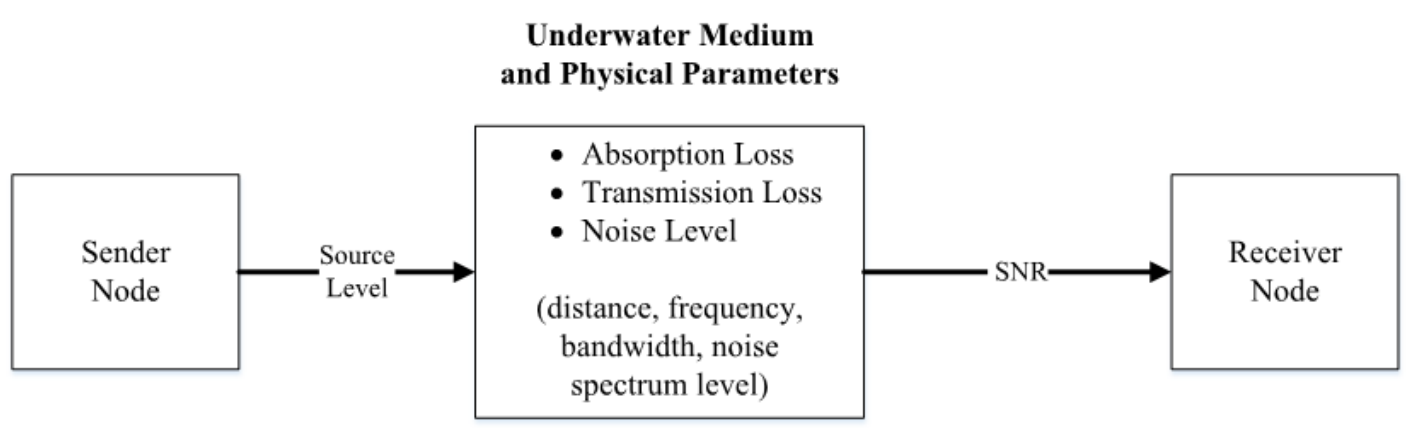

Figure 5.1: Simulator communication model

There are mainly four simulation scenarios in this chapter. The common simulation parameters are given in Table 5.1.

Table 5.1: Simulation parameters

\begin{tabular}{|l|l|}
\hline Topology area & $1000 \mathrm{~m}^{*} 1000 \mathrm{~m}$ \\
\hline Topology depth & $200 \mathrm{~m}$ \\
\hline $\begin{array}{l}\text { Transmitting Voltage Response } \\
(\mathrm{TVR})\end{array}$ & $95 \mathrm{~dB}$ \\
\hline Transmission voltage & $390 \mathrm{Vrms}$ \\
\hline Noise Spectrum Level & $40 \mathrm{~dB}$ \\
\hline Frequency band & $10 \mathrm{kHz}-18 \mathrm{kHz}$ \\
\hline Bandwidth & $8 \mathrm{kHz}$ \\
\hline Bit rate & $1 \mathrm{kbit} / \mathrm{s}$ \\
\hline Packet size & $10 \mathrm{Bytes}$ \\
\hline Node Buffer Time & $10 \mathrm{msec}$ \\
\hline
\end{tabular}

The source level and the noise level are obtained from related equations in Chapter 3.

Source Level $=139.54 \mathrm{~dB}$

Noise Level $=79.03 \mathrm{~dB}$ 
All simulations are performed for the new routing protocol TC-VBF. It will be compared with the classical VBF and the hop-by-hop VBF (HH-VBF) approaches according to network parameters with different simulation scenarios.

The simulation scenarios are described below and the results related to the simulations will be given and evaluated.

\subsubsection{Comparison Metrics}

Performance comparison between algorithms in this work includes packet delivery ratio, delay performance, retransmission performance and packet fail rates. There are 4-graph outputs in MATLAB underwater channel simulator for each simulation topology in this work: packet delivery ratio graph, average end-to-end delay graph, average number of retransmission graph, and histogram of retransmission numbers. The packet delivery ratio graph shows a ratio of successful transmitted packets to all packets for a first transmission attempt. Moreover, the average end-to-end delay graph is obtained from averaging delay values of each successful packet transmissions. Likewise, the average number of transmission graph is obtained from averaging transmission numbers of each successful packet transfer. Furthermore, the histogram of transmission numbers shows a percentage distribution of transmission numbers of each successful attempt. The histogram of transmission numbers shows also percentage of fail packets.

At the end of simulations, there is a failure rate table. It shows failure packet rates of protocols. 


\subsection{Performance Evaluation}

\subsubsection{Selection of Modulation Technique}

Firstly, modulation technique used in the simulations is investigated. Binary PhaseShift Keying (BPSK) and Quadrature Phase-Shift Keying (QPSK) are simulated according to different SNR values under water conditions.

In simulations, both signal-plus-noise and noise are assumed to have a Gaussian distribution.

The graph in Figure 5.2 shows a packet error probability of BPSK and QPSK versus SNR. For high SNR values, both techniques have a low packet error rates. On the other hand, for low SNR values, BPSK have a better packet error performance than QPSK.

Since a successful transmission between long distances is desired, BPSK seems to be more suitable although higher bit rate can be achieved with QPSK. Therefore, BPSK technique is selected to use in this work. The packet probabilities in Figure 5.2 are used in simulations according to related SNR values. 


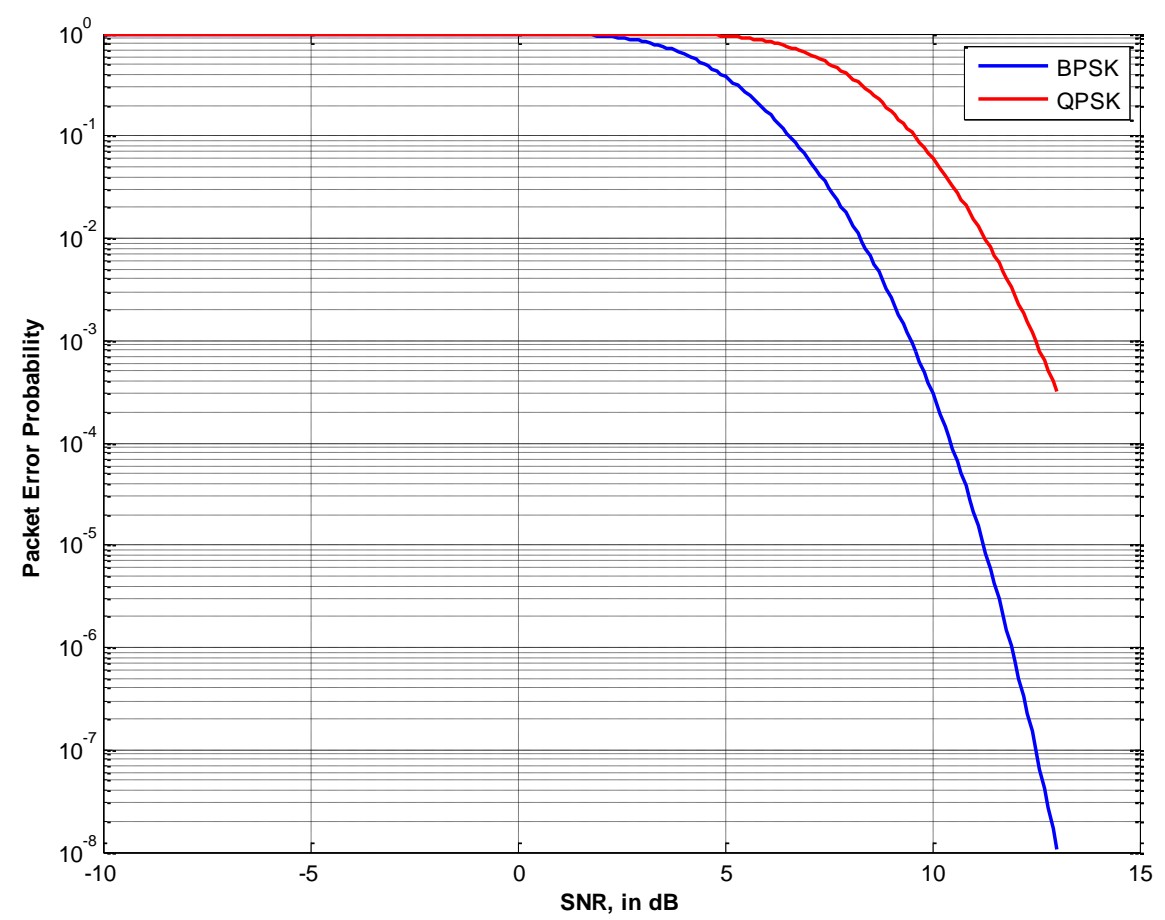

Figure 5.2: Packet Error Probability vs. SNR

\subsubsection{Performance Comparison for Topology 1}

The first simulation is the comparison of performance for routing protocols. The TCVBF is compared with the VBF and the HH-VBF protocols. For this simulation, the infrastructure can be seen in Figure 5.3. The simulation scenario is described as follows:

- There are 6 nodes. Only one node is a source node. The distance between the source node and the sink node, $\mathrm{d} 1$, is greater than the threshold transmission distance and less than two times the threshold transmission distance. Moreover, the distance $\mathrm{d} 2$ between the source node and the sub-node participated in the packet transfer is less than the threshold distance and the distance $\mathrm{d} 3$ between this sub-node and the sink node is less than the threshold distance. The distance $\mathrm{d} 1$ is varying $450 \mathrm{~m}$ to $650 \mathrm{~m}$. 
- The threshold distance for TC-VBF is set to $500 \mathrm{~m}$. Parameter $\mathrm{N}$ for TC-VBF is set to 2 .

- Simulation parameter settings are the same in Table 5.1.

- Each data point in the graphs was obtained by averaging of 100 runs.

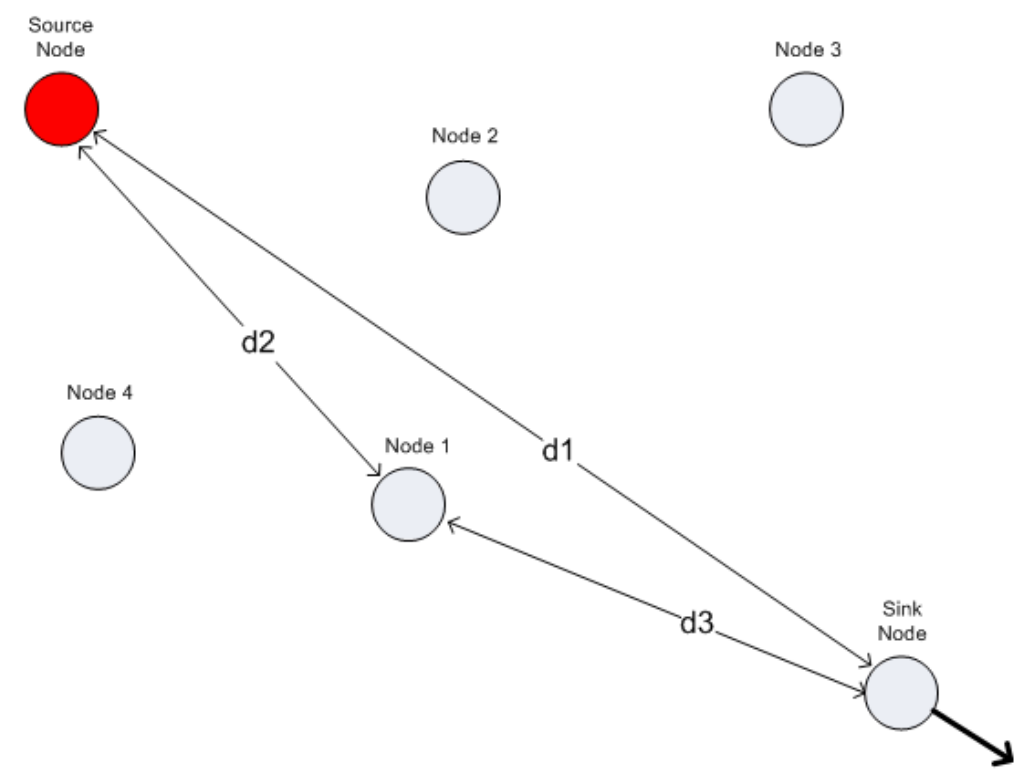

Figure 5.3: Simulation topology 1 


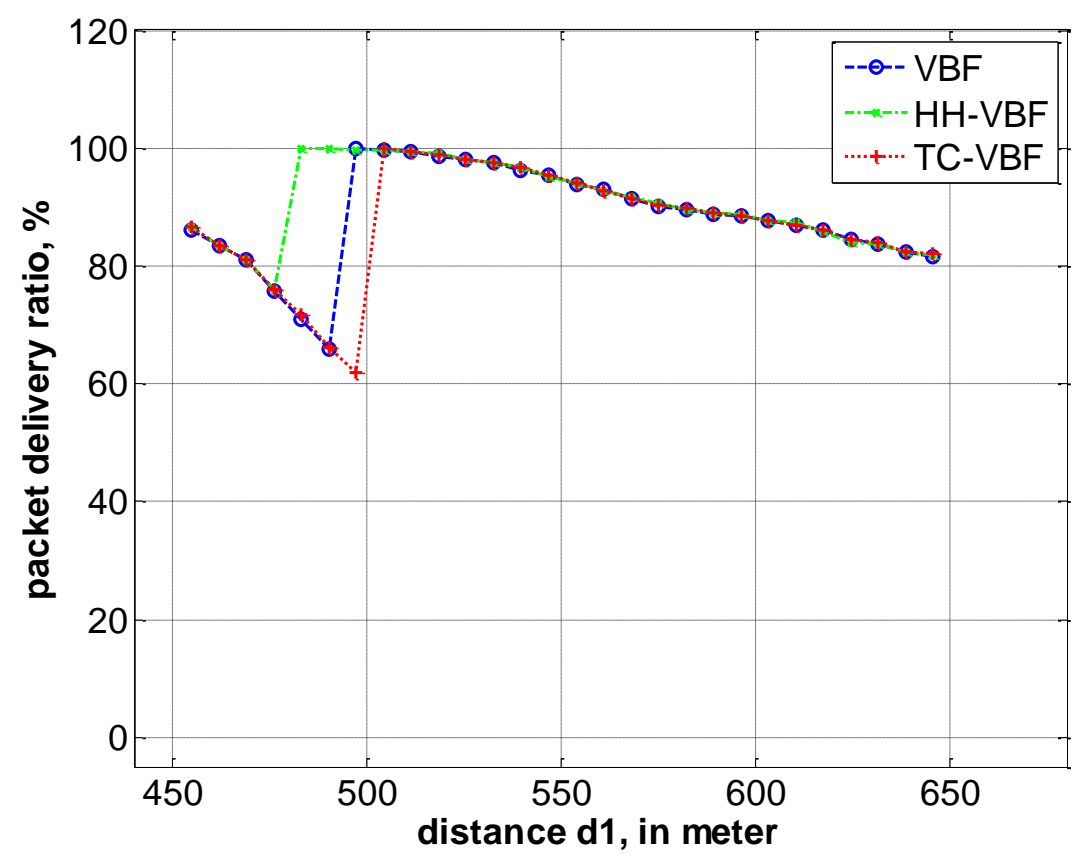

Figure 5.4: Packet delivery ratio graph for scenario 1

When the graph in Figure 5.4 is investigated, protocols have a rapid increase in packet delivery ratio at different distances since they have different relay algorithm. In the TC-VBF and the VBF, packet delivery ratio is decreasing 84 percent to 60 percent when the distance $\mathrm{d} 1$ goes to $500 \mathrm{~m}$ whereas the packet delivery ratio is increasing from 80 percent to 100 percent at distance $465 \mathrm{~m}$. On the other hand, when the distance $\mathrm{d} 1$ is between $500 \mathrm{~m}$ and $650 \mathrm{~m}$, packet delivery ratios for all protocols have greater than 80 percent. The reason of that data packets are forwarded by the forwarder node, Node 1, for all protocols. Node 1, inside the vector from source to sink, relays the data packets to the sink node according to all algorithms. 


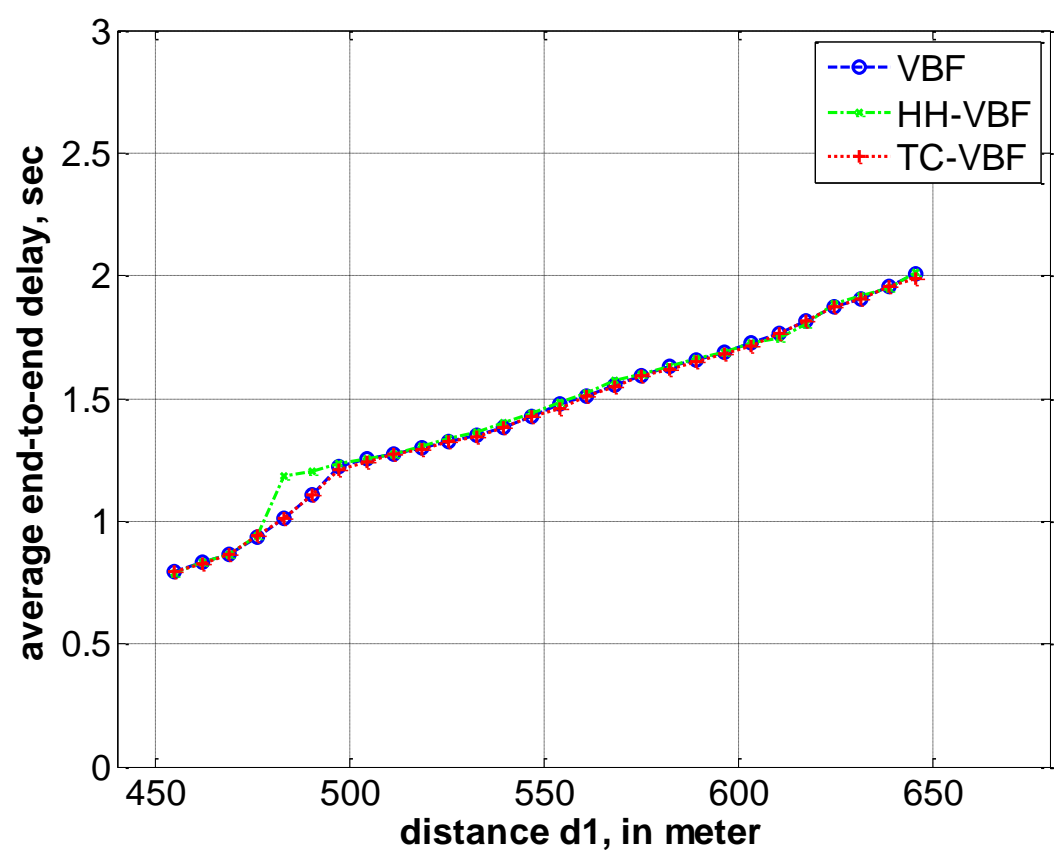

Figure 5.5: Average end-to-end delay graph for scenario 1

The graph in Figure 5.5 illustrates that all of three algorithms have the same end-toend delay performance for data packets although there are small differences at distances lower than $500 \mathrm{~m}$. Average end-to-end delay is roughly between 0.8 and 2 seconds and it is increasing with increasing distance $\mathrm{d} 1$. On the other hand, when it is focused on the distance which algorithm starts to relay data, there is a relatively high increase. 


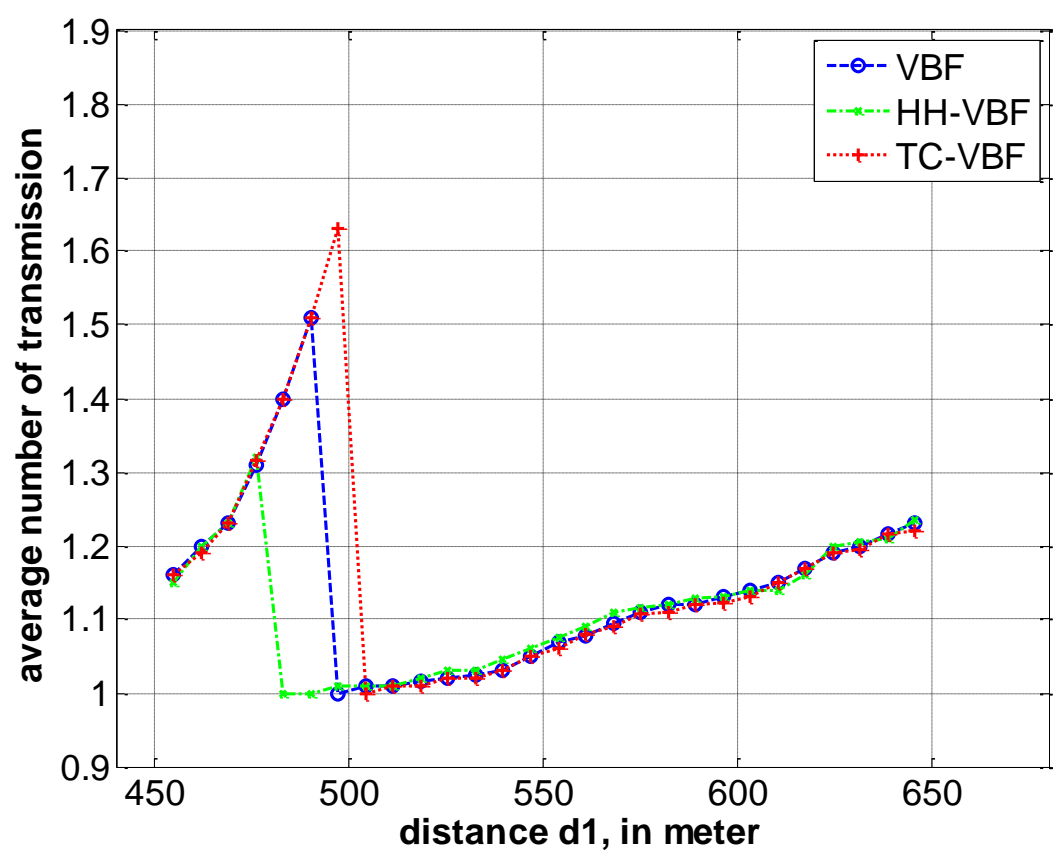

Figure 5.6: Average number of transmission graph for scenario 1
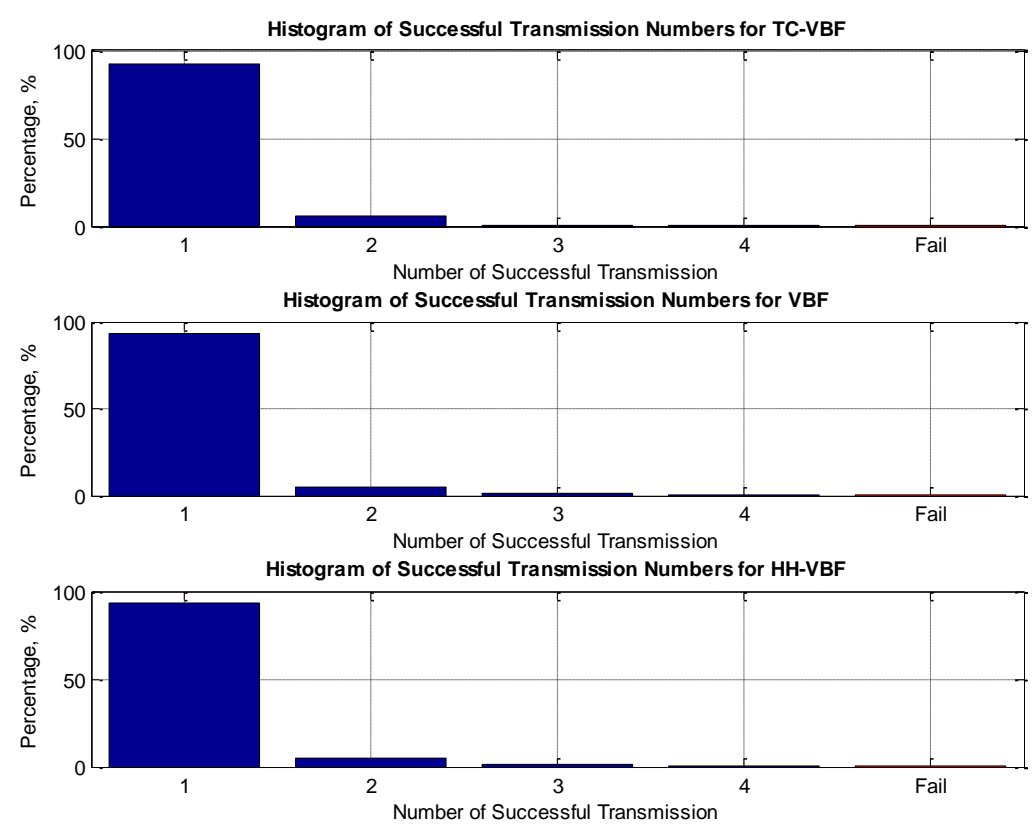

Figure 5.7: Histogram of successful transmission numbers for scenario 1 
Table 5.2: Packet failure rate table for scenario 1

\begin{tabular}{|c|c|}
\hline Protocol & Failure Rate (\%) \\
\hline TC-VBF & 0.08 \\
\hline VBF & 0.09 \\
\hline HH-VBF & 0.06 \\
\hline
\end{tabular}

According to graphs in Figure 5.6 and Figure 5.7, all of three protocols have close retransmission performance for this topology and simulation. When the distance is below the $500 \mathrm{~m}$, average number of retransmission is increasing rapidly for all three algorithms. On the contrary, the average number of retransmission is considerably close to 1 for the distance greater than $500 \mathrm{~m}$ because of relaying data packet with the help of forwarder node. In addition, packet failure rates are very small from the Table 5.2.

\subsubsection{Performance Comparison for Topology 2}

The second simulation is also a comparison of performance for routing protocols for different topology. The TC-VBF is compared with the VBF and the HH-VBF routing protocols. For this simulation, the topology is based on tactical plan for ASELSAN ZOKA ${ }^{\mathrm{TM}}$ project. The infrastructure can be seen in Figure 5.8. The simulation scenario is described as follows:

- There are 7 nodes. Only one node is a source node. All nodes are at different directions according to the position of the source node. The distance between the source node and the sink node $\mathrm{d}$ is varying $450 \mathrm{~m}$ to $650 \mathrm{~m}$. Node 5 is also moving while the sink node is moving.

- The threshold distance for TC-VBF is set to $500 \mathrm{~m}$. The parameter $\mathrm{N}$ for the TC-VBF is set to 2 . The node velocity is around $5 \mathrm{~m} / \mathrm{sec}$.

- Simulation parameter settings are the same in Table 5.1. 
- Each data point in the graphs was obtained by averaging of 100 runs.

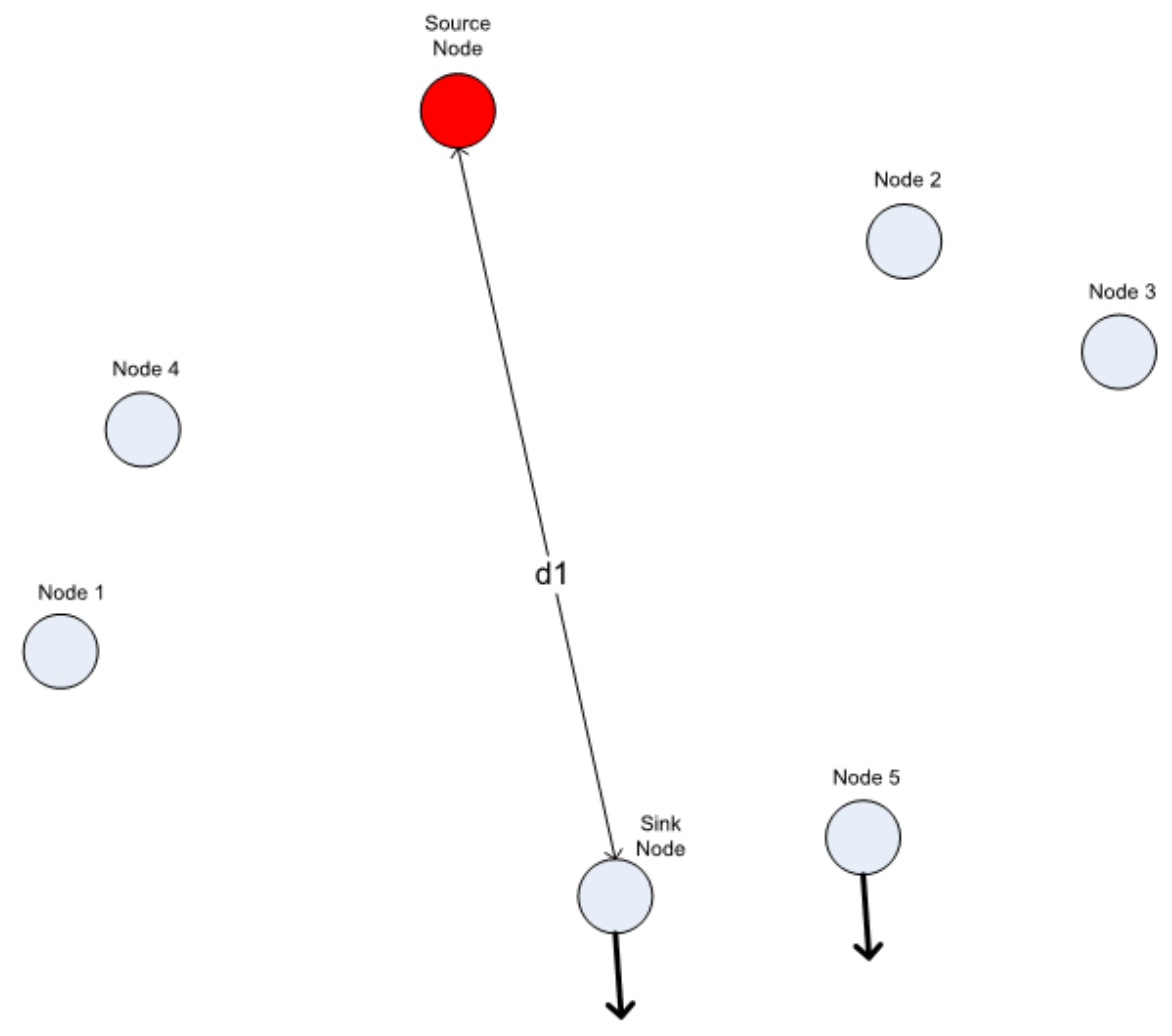

Figure 5.8: Simulation topology 2

When the Figure 5.9 is analysed, all protocols have the same packet delivery ratio for distances below $500 \mathrm{~m}$. However, when the distance $\mathrm{d} 1$ is greater than $500 \mathrm{~m}$, the TC-VBF transmits nearly all packets for the first attempt whereas packet delivery ratio is decreasing to 10 percent for both the VBF and the HH-VBF. The reason is that the distance between the sink node and the forwarder node is increasing according to the VBF and the HH-VBF algorithm when the distance d becomes greater than 500m and SNR is decreasing. On the other hand, the TC-VBF algorithm uses an actor node in the packet transfer. The actor node moves to the midpoint of the distance between the source node and the sink node and the TC-VBF does not let SNR to decrease. Thus, it realizes the data transmission with high successful rate. 


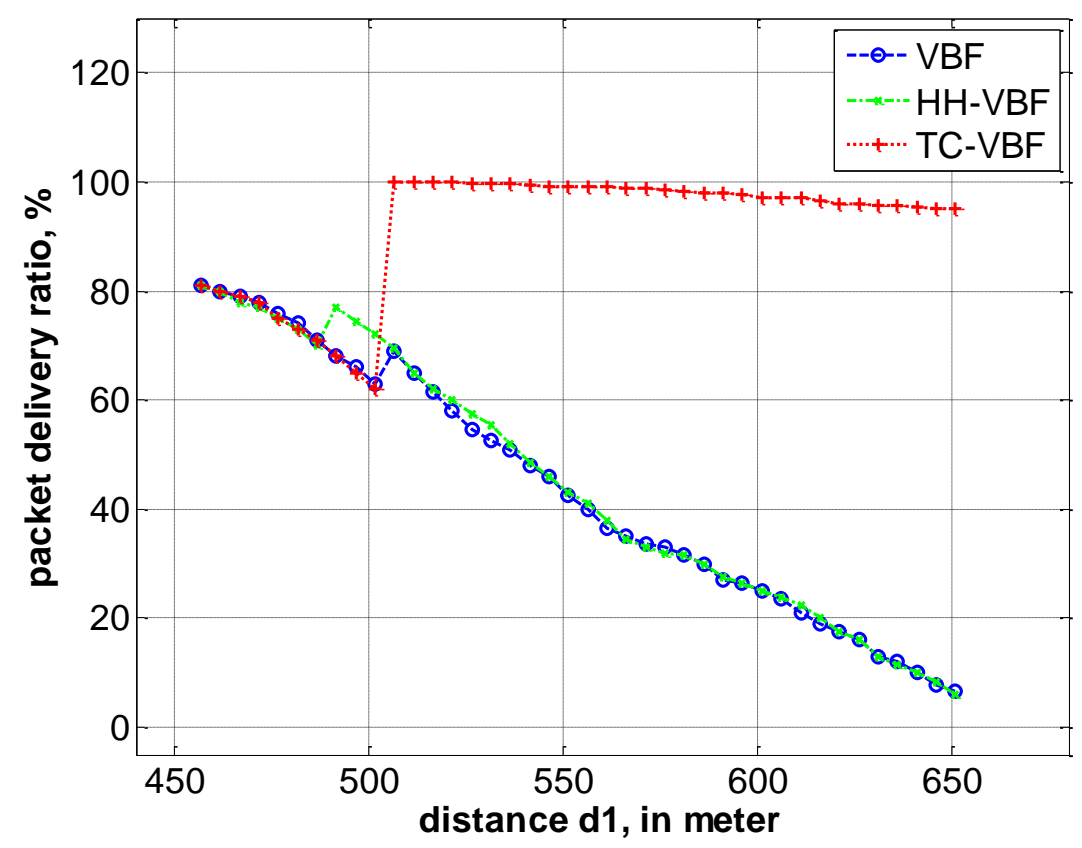

Figure 5.9: Packet delivery ratio graph for scenario 2

The average end-to-end delay graph in Figure 5.10 shows that an average end-to-end delay is the same and around 1 second for all three protocols at distances lower than $500 \mathrm{~m}$. End-to-end delay values for the VBF and the HH-VBF have an increase, but not great, as data is relayed at this distance. On the other hand, end-to-end delay values for the TC-VBF approach have a rapid increase at the distance $500 \mathrm{~m}$ and they increase to 23 seconds because there is a node movement to transmit packets successfully and it takes long time. 


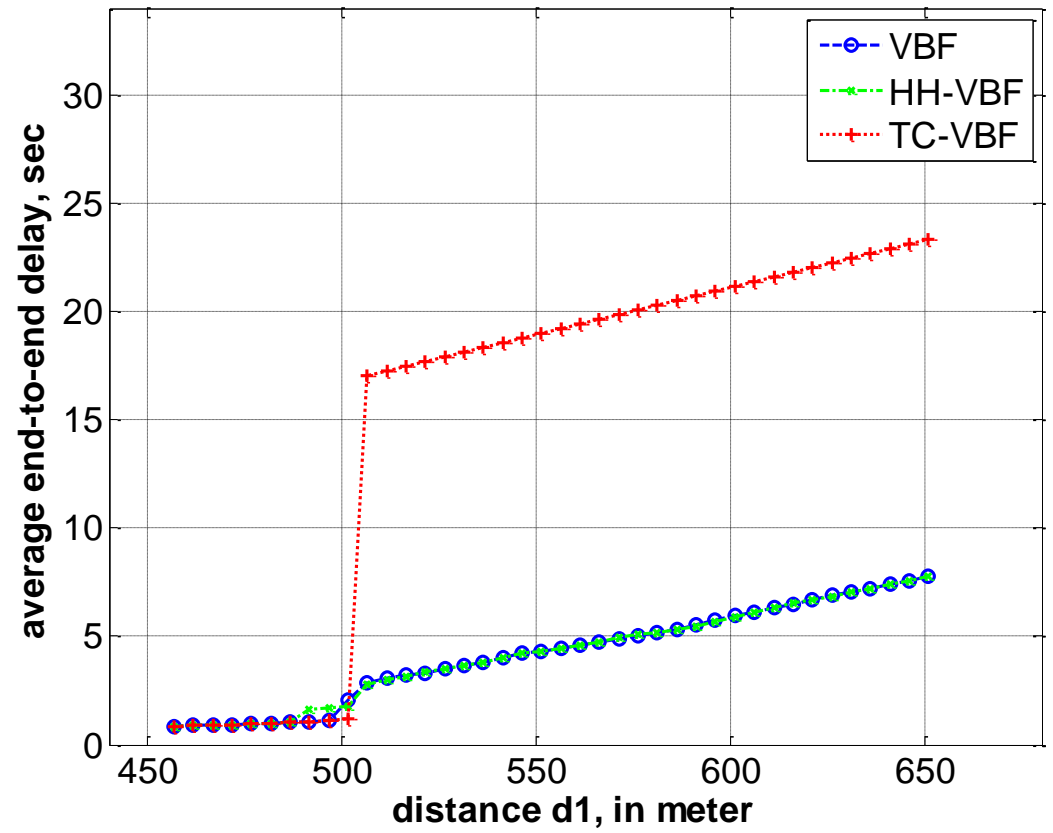

Figure 5.10: Average end-to-end delay graph for scenario 2

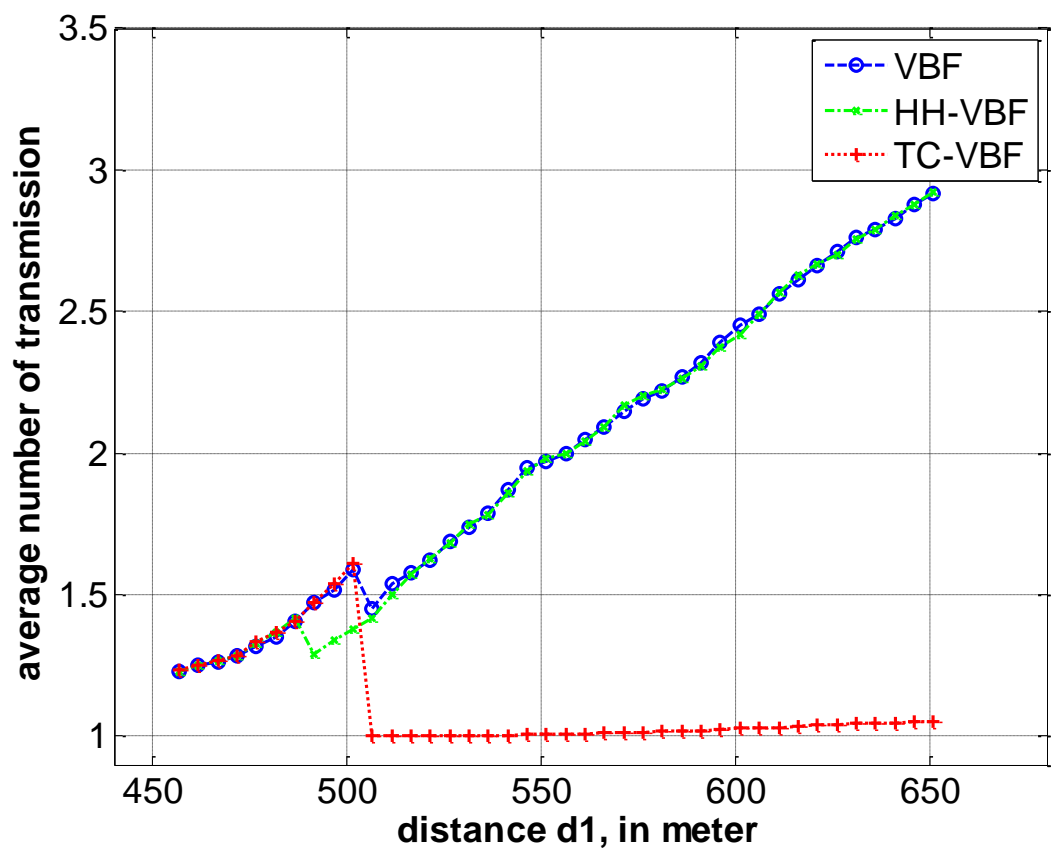

Figure 5.11: Average number of retransmission graph for scenario 2 
According to the graph in Figure 5.11, the HH-VBF have a better performance than the VBF and the TC-VBF algorithms at distances lower than $500 \mathrm{~m}$ because the $\mathrm{HH}-$ $\mathrm{VBF}$ relays data packets before both the VBF and the TC-VBF. On the other hand, the average number of retransmission for the $\mathrm{TC}-\mathrm{VBF}$ is decreasing to 1 and keeps constant with increasing distance while this number for both the VBF and the HH$\mathrm{VBF}$ is increasing to 3 . Therefore, the $\mathrm{TC}-\mathrm{VBF}$ has a better overall retransmission performance than the others for this simulation scenario.
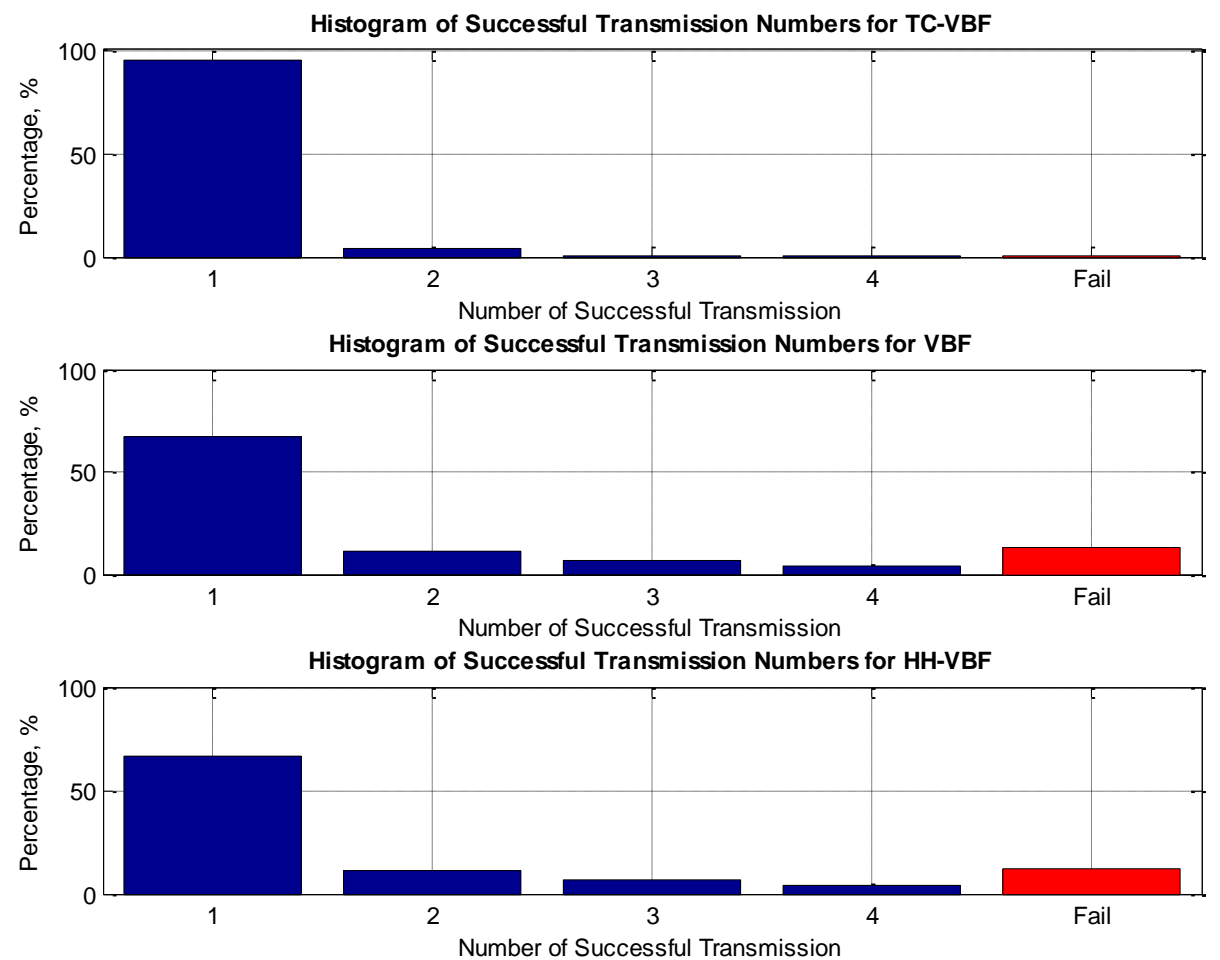

Figure 5.12: Histogram of retransmission numbers for scenario 2

Table 5.3: Packet failure rate table for scenario 2

\begin{tabular}{|c|c|}
\hline Protocol & Failure Rate (\%) \\
\hline TC-VBF & 0.11 \\
\hline VBF & 12.49 \\
\hline HH-VBF & 11.24 \\
\hline
\end{tabular}


When the histogram of retransmissions in Figure 5.12 is investigated, TC-VBF transmits data packets mostly for the first attempt and all data packets are successfully transferred at the end, whereas 2, 3 and 4 retransmission numbers can be seen for the VBF and the HH-VBF. Furthermore, they have around 12 percent packet failure rate from the Table 5.3.

\subsubsection{Performance Comparison for Long Range with $\mathrm{N}=2$}

Another simulation has almost the same network topology with the simulation scenario 1. For this simulation, the infrastructure can be seen in Figure 5.13. The simulation scenario is described as follows:

- There are also 6 nodes. The only difference with topology 2 is the distance between the sub-node and the sink node, $\mathrm{d} 3$. The distance $\mathrm{d} 3$ is greater than the maximum transmission distance. The distance $\mathrm{d} 1$ is varying $650 \mathrm{~m}$ to $950 \mathrm{~m}$.

- The threshold distance for TC-VBF is set to $500 \mathrm{~m}$. The parameter $\mathrm{N}$ for the TC-VBF is set to 2 . The node velocity is around $5 \mathrm{~m} / \mathrm{sec}$.

- Simulation parameter settings are the same in Table 5.1.

- Each data point in the graphs was obtained by averaging of 100 runs. 


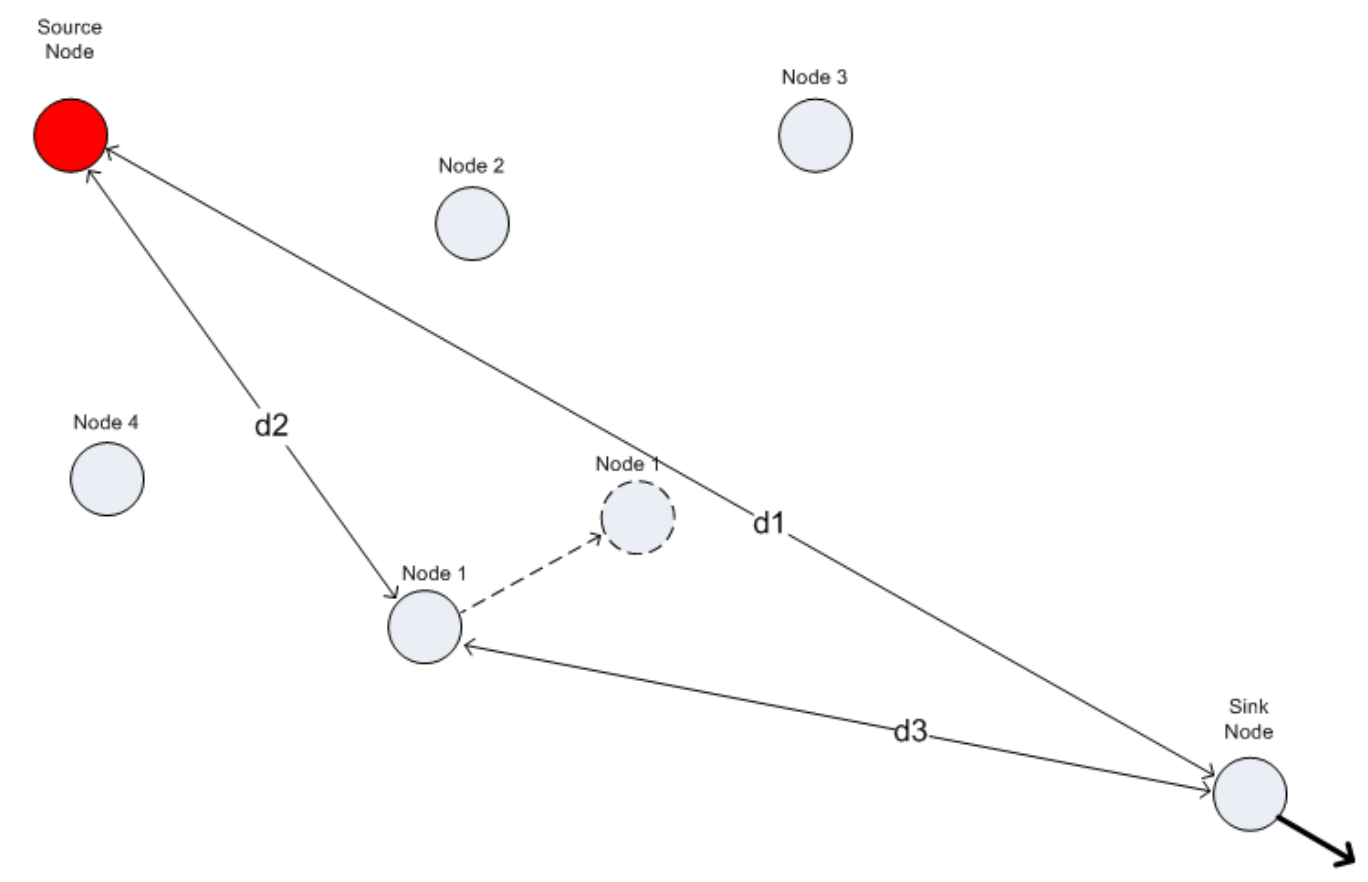

Figure 5.13: Simulation topology 3

The graph in Figure 5.14 shows that the packet delivery ratio for the TC-VBF is increases 100 percent at distance $680 \mathrm{~m}$ since a node movement occurs. In the TC$\mathrm{VBF}$, the actor node helps the packet transfer by moving to the midpoint of the distance $\mathrm{d} 1$. Thus, data packets are delivered for long ranges with the help of the TC-VBF algorithm. On the other hand, according to the VBF and the HH-VBF algorithms, a packet delivery ratio is decreasing to 0 as forwarder node does not move for these algorithms and the sink node is moving away from the forwarder node. Hence, SNR is decreasing and it affects successful packet transfer negatively. 


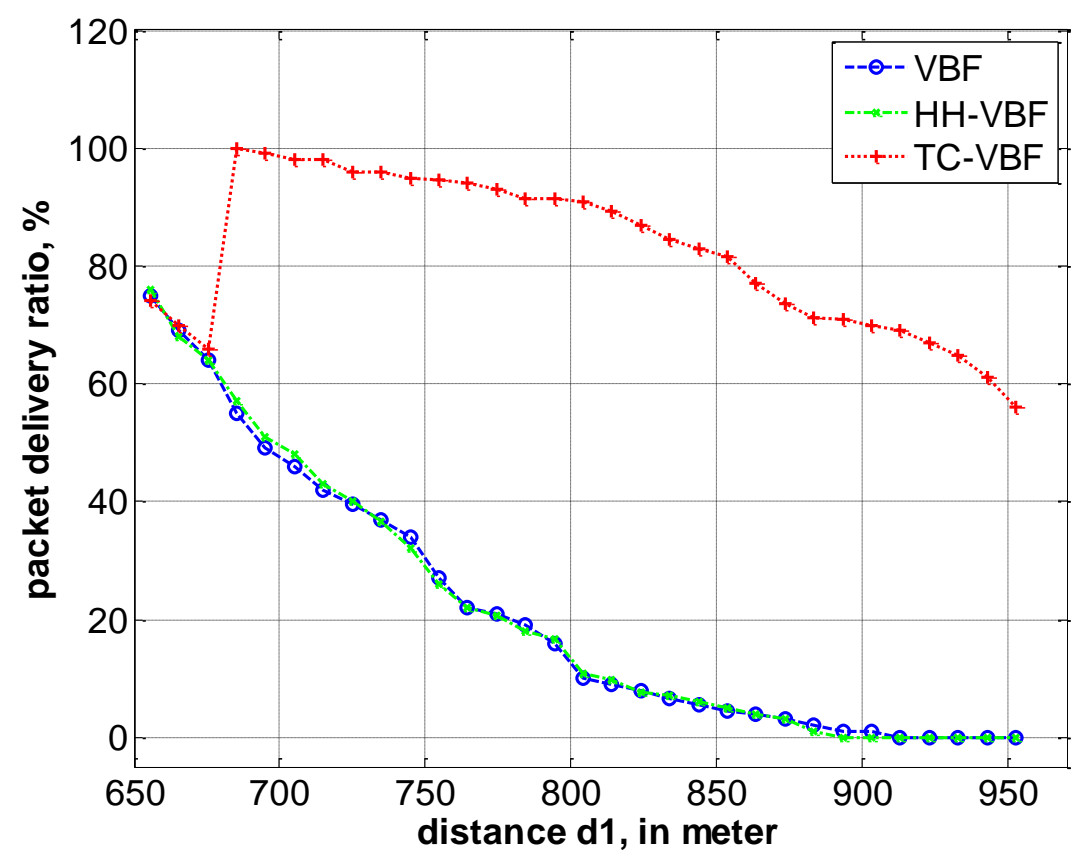

Figure 5.14: Packet delivery ratio graph for scenario 3

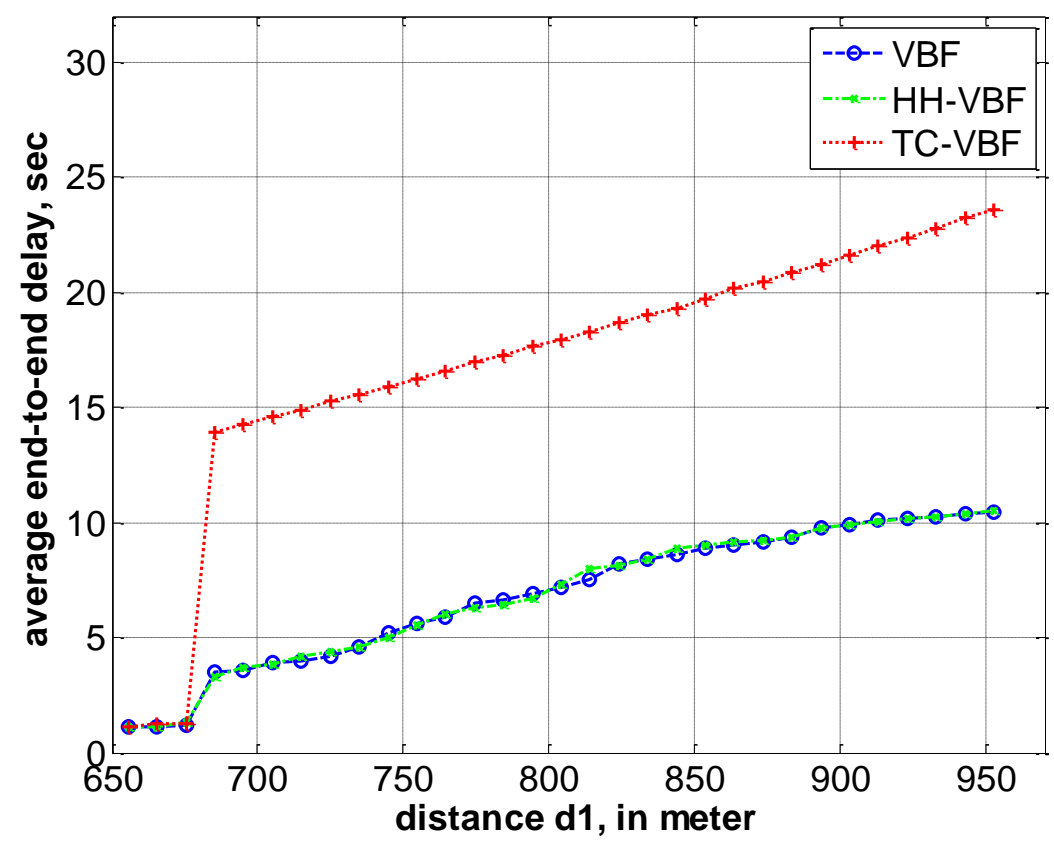

Figure 5.15: Average end-to-end delay graph for scenario 3 
The average end-to-end delay graph for the scenario 3 can be seen in Figure 5.15. According to the graph, end-to-end delay for the TC-VBF is rapidly increasing to 14 seconds at distance $670 \mathrm{~m}$ since the node movement is required and it takes long time. After that, it continues to increase with the propagation delay. On the other hand, although the end-to-end delay performance of the VBF and the HH-VBF are seen better, they cannot transmit packets successfully for long distances.

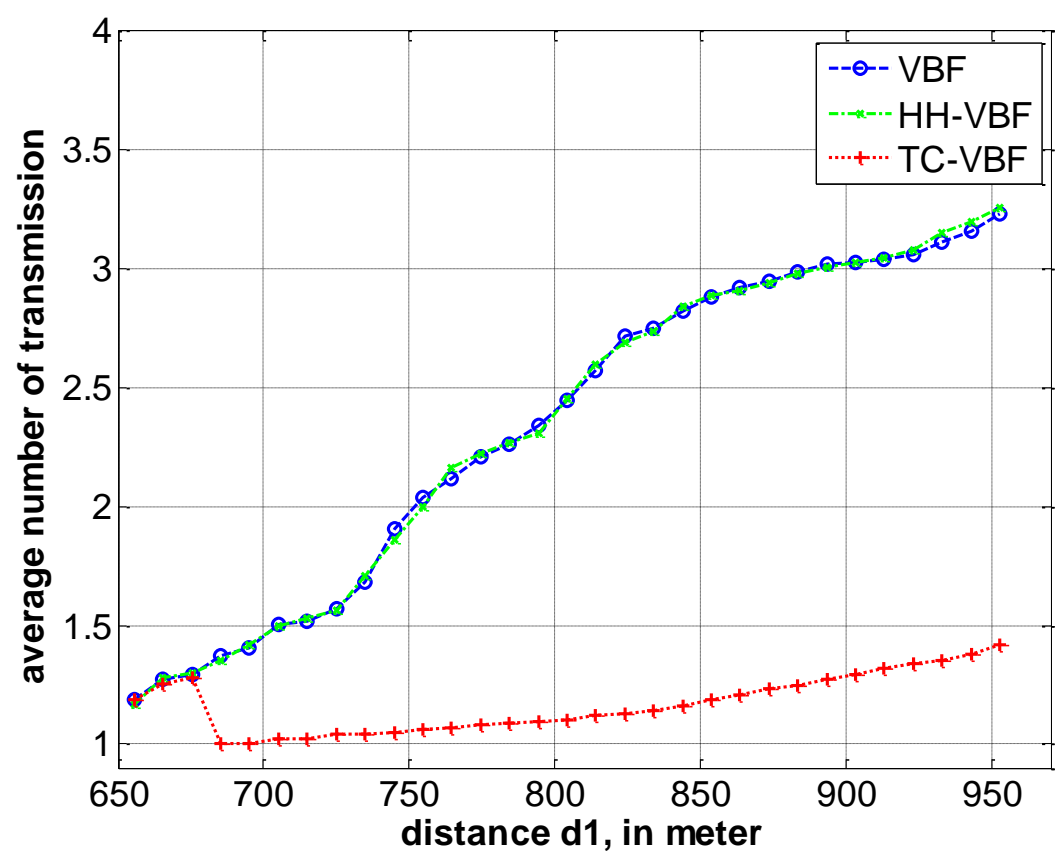

Figure 5.16: Average number of retransmission graph for scenario 3

The average number of retransmission graph in Figure 5.16 illustrates that retransmission numbers of the TC-VBF algorithm is below 1.5 and they are nearly to 1 for distances between $680 \mathrm{~m}$ and $850 \mathrm{~m}$, whereas these numbers of the VBF and the $\mathrm{HH}-\mathrm{VBF}$ are increasing to 3 with increasing the distance since the sink node is moving away from both the source node and the forwarder node. 

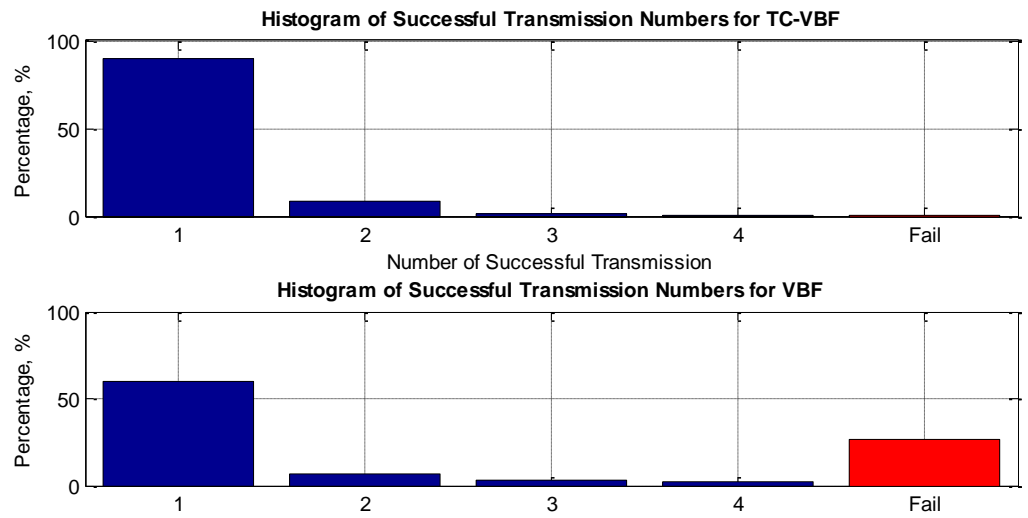

Number of Successful Transmission

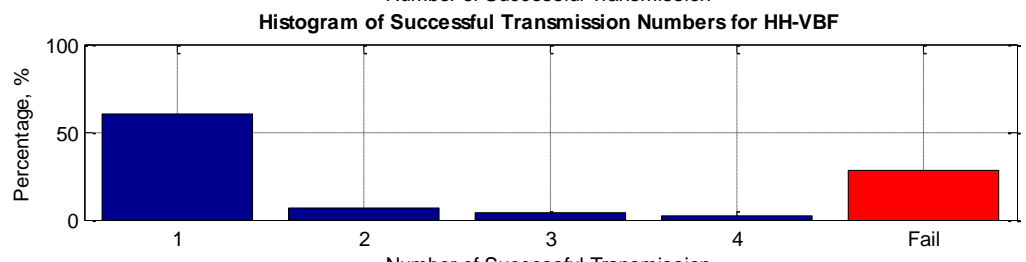

Number of Successful Transmission

Figure 5.17: Histogram of retransmission numbers for scenario 3

Differences between algorithms according to number of successful first attempts can easily be seen in Figure 5.17. The TC-VBF realizes above 90 percent success rate for the first attempt while there are around 50 percent success rate for the VBF and the HH-VBF. Moreover, number of failed packets for both the VBF and the HHVBF are considerably higher than the TC-VBF. According to Table 5.4, failure rate is around 27 percent for the VBF and the HH-VBF.

Table 5.4: Packet failure rate table for scenario 3

\begin{tabular}{|c|c|}
\hline Protocol & Failure Rate (\%) \\
\hline TC-VBF & 0.19 \\
\hline VBF & 27.63 \\
\hline HH-VBF & 26.11 \\
\hline
\end{tabular}




\subsubsection{Performance Comparison for Long Range with $\mathrm{N}=3$}

The previous simulation is repeated with different $\mathrm{N}$ value of the TC-VBF algorithm. For this simulation, the infrastructure is the same with the previous simulation topology. The simulation scenario is described as follows:

- There are also 6 nodes. The distance $\mathrm{d} 1$ is varying $950 \mathrm{~m}$ to $1500 \mathrm{~m}$.

- The threshold distance for the TC-VBF is set to $500 \mathrm{~m}$. The parameter $\mathrm{N}$ for TC-VBF is set to 3 . The node velocity is around $5 \mathrm{~m} / \mathrm{sec}$.

- Simulation parameter settings are the same in Table 5.1.

- Each data point in the graphs was obtained by averaging of 100 runs.

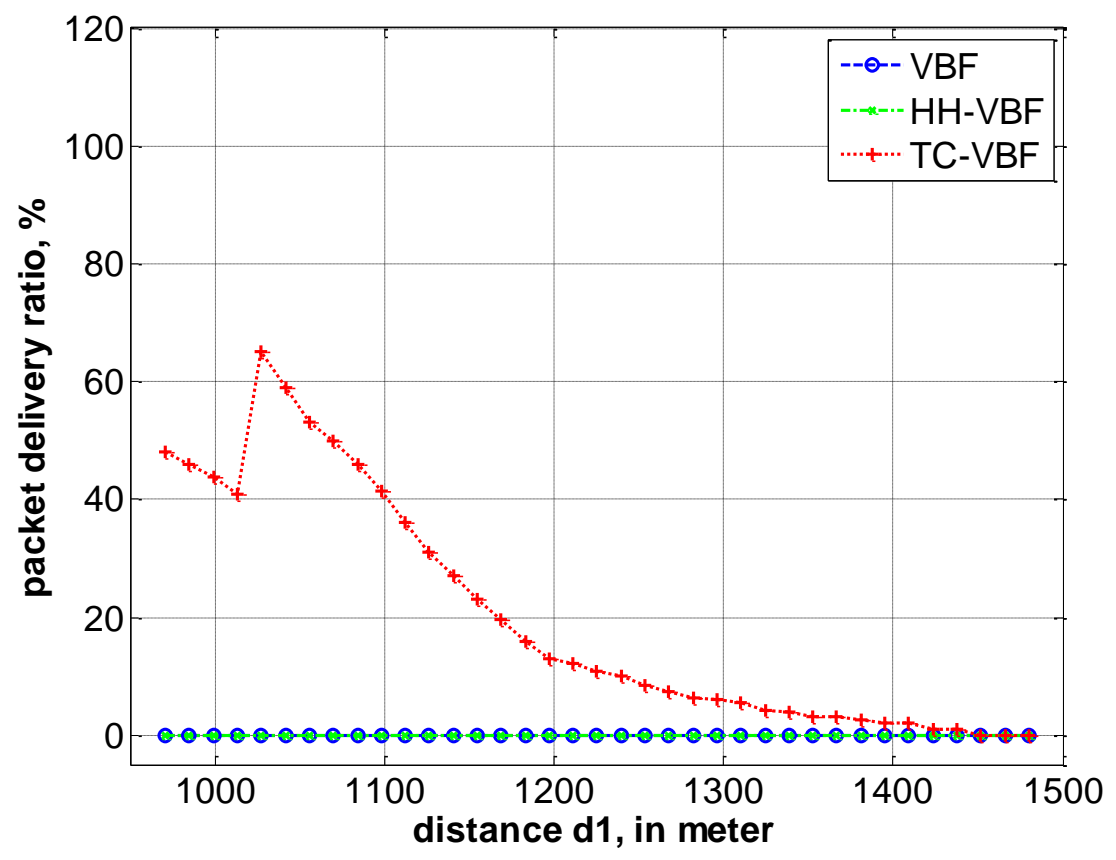

Figure 5.18: Packet delivery ratio graph for scenario 4

The packet delivery ratio graph for scenario 4 can be seen in Figure 5.18. For this topology, there is no successful data transfer in the VBF and the HH-VBF algorithm for a first transmission as the distance is highly great and SNR is low. On the other hand, according to the TC-VBF algorithm, the packet delivery ratio is around 50 
percent and it is decreasing to 0 with increasing distance. Actor node or nodes participate in the data transmission for the TC-VBF algorithm and they increase the packet delivery ratio.

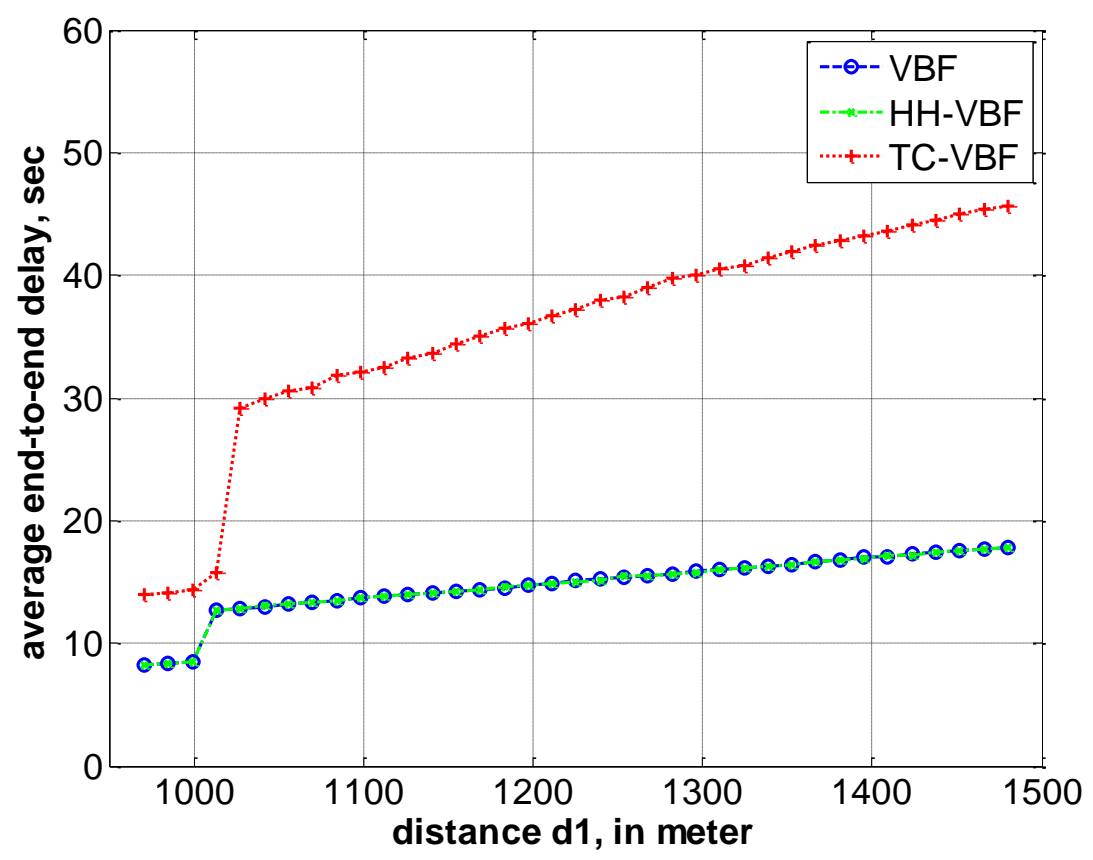

Figure 5.19: Average end-to-end delay graph for scenario 4

The graph above in Figure 5.19 is the average end-to-end delay graph for this simulation scenario. According to the graph, an end-to-end delay value of the TCVBF is increasing from 15 seconds to 45 seconds with increasing frequency because of the node movement. On the other hand, delay values for the VBF and the $\mathrm{HH}_{-}$ VBF is lower than that for the TC-VBF since node movements take long time in the TC-VBF algorithm and only small portion of packets are successfully transmitted in the VBF and the HH-VBF algorithms. 


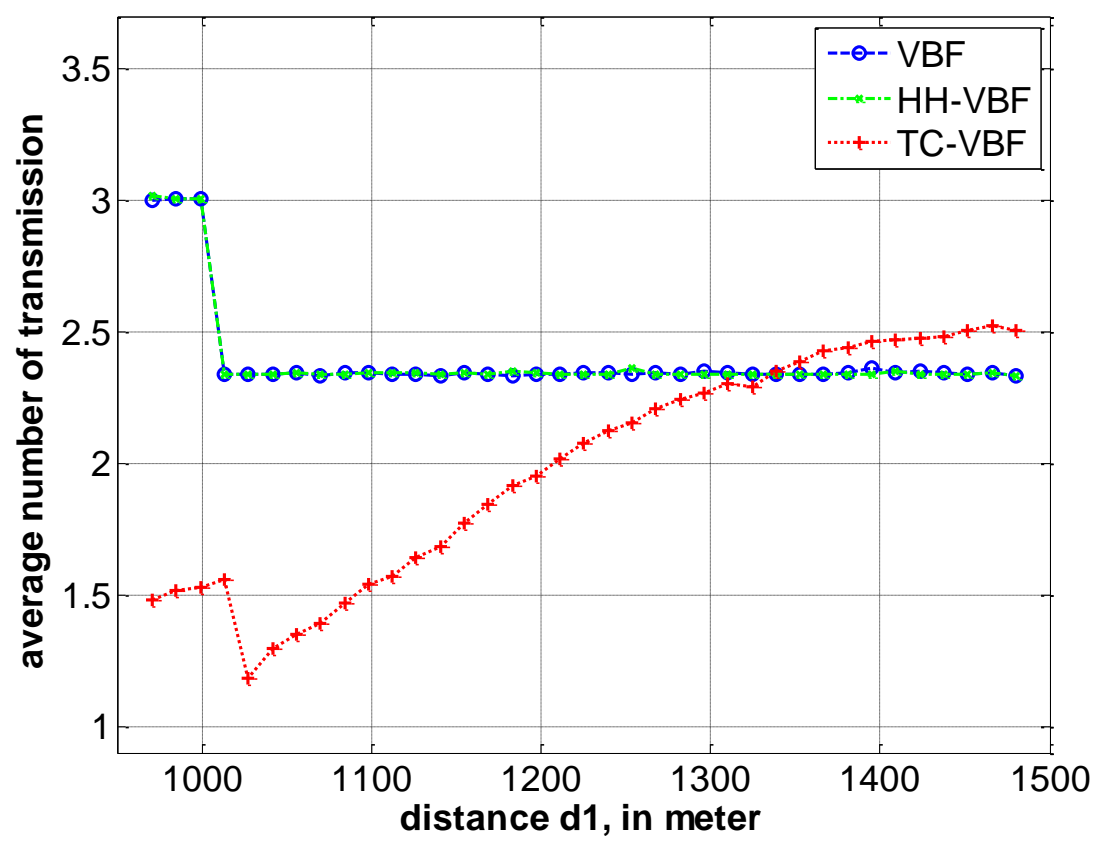

Figure 5.20: Average number of retransmission graph for scenario 4

The figure 5.20 shows that an average number of retransmission for the TC-VBF is less than the others for distance d 1 lower than $1350 \mathrm{~m}$, whereas this is more than the others for $\mathrm{d} 1$ greater $1350 \mathrm{~m}$. The reason is that small number of packets is transmitted with the VBF and the HH-VBF protocols for long distances and only successful transmitted packets are included in this graph. Moreover, the average number of retransmission for the VBF and the HH-VBF is seen constant after the distance $1000 \mathrm{~m}$ since SNR is very low for those distances and the packet delivery ratio stays constant. 

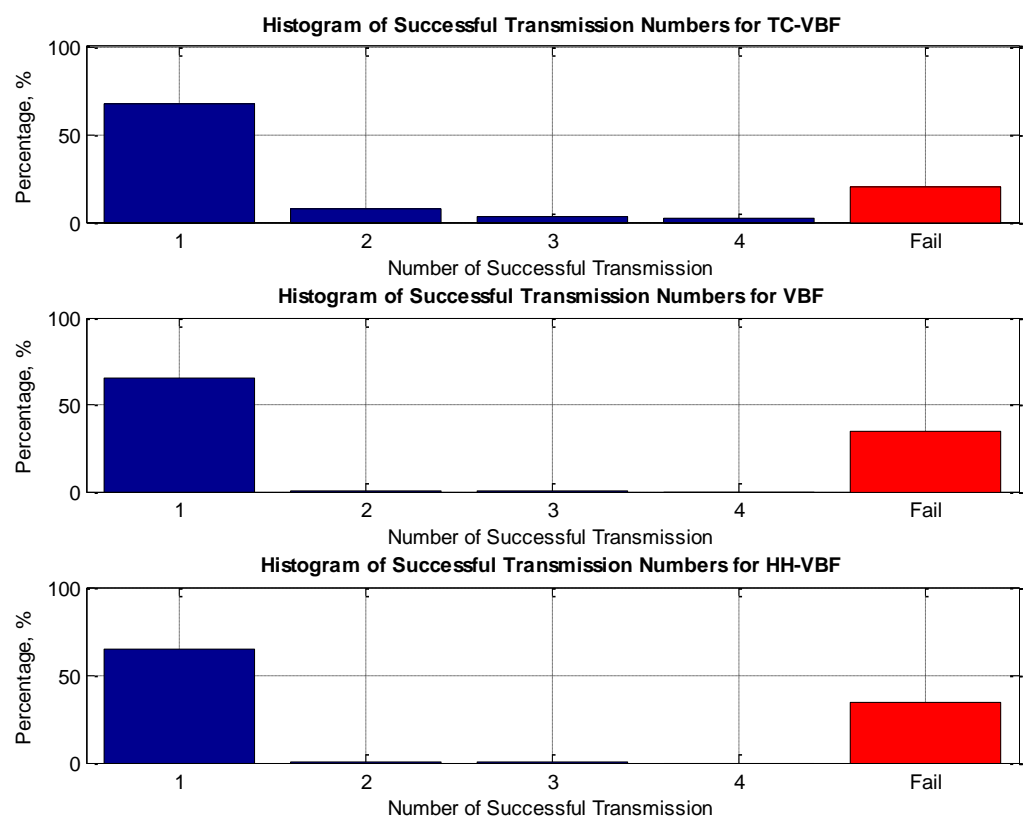

Figure 5.21: Histogram of retransmission numbers for scenario 4

Table 5.5: Packet failure rate table for scenario 4

\begin{tabular}{|c|c|}
\hline Protocol & Failure Rate (\%) \\
\hline TC-VBF & 15.94 \\
\hline VBF & 34.82 \\
\hline HH-VBF & 34.17 \\
\hline
\end{tabular}

According to the graph in Figure 5.21, when the number of successful transmission for the first attempt is observed, the TC-VBF algorithm outperforms the others. On the other hand, the VBF and the HH-VBF have more number of packets failed in transmission than the TC-VBF. Moreover, Table 5.5 illustrates the failure rates of algorithms for this simulation scenario. 


\subsection{Conclusion}

The simulations performed in previous parts show that the TC-VBF algorithm has a better performance compared to the classical VBF and the HH-VBF for underwater acoustic networks. For close distance, all three protocols work similar and the average number of retransmission, the packet delivery ratio and the average end-toend delay values are almost the same. On the other hand, when the distance between the source and the sink nodes is increased, the HH-VBF and the VBF algorithms could not satisfy the packet transmission to the sink node for the first transmission whereas the TC-VBF approach transfers the data with the help of the actor node's movement with better transmission rate. For the TC-VBF, the parameter $\mathrm{N}$ is important for the maximum communication range. While $\mathrm{N}$ is increasing, the communication range is also increasing. However, the end-to-end delay is rapidly increasing for the TC-VBF since the movement of actor node or nodes to the midpoints takes much time and it is a dominant factor for the this protocol.

The TC-VBF approach has lower packet failure rate than the others for all simulation scenarios. Especially for long ranges, the packet failure rates for the VBF and the HH-VBF algorithms are high.

As a result, in the light of information from simulation results, if the reliable data transfer for long ranges is aimed for UASN with the small number of nodes, it is clear that the TC-VBF approach is a promising solution. 


\section{CHAPTER 6}

\section{CONCLUSION AND FUTURE WORK}

Underwater environment has high absorption rate, where the bandwidth is limited, propagation delay is large, and time variation is rapid. Due to these challenges, underwater communication networks do not have a long communication range. High node dense may increase the communication range for UASN with proper protocols and algorithms. However, when the networks with small number of nodes are considered, the communication range could not be as long as desired. In the ASELSAN's underwater communication network project, the long communication range is needed. To ensure this requirement, the novel paradigm, the TC-VBF, is proposed and developed.

Underwater acoustic channel characteristics are meticulously investigated. Moreover, existing MAC, transport layer, routing layer protocols and topology control algorithms are classified and reviewed. Advantages and disadvantages of them for the UASN are discussed in depth. Most of protocols used in terrestrial networks are claimed to be inapplicable for underwater acoustic networks because of the underwater medium. Moreover, the technical analysis for underwater communication is carried out in this work. The maximum transmission range formula is obtained and analyzed.

The TC-VBF, newly proposed algorithm for underwater sensor networks, is location-based, centralized topology control approach in order to enhance the communication range performance for networks. It provides scalable, robust and reliable data transmission. According to the TC-VBF, the master node initializes the data transfer and all data packets are sent from this node. Firstly, the master node calculates the distance to the sink node. If this distance is lower than the threshold 
distance, data packets are directly transmitted. If it is not, then it calculates distances between other nodes in the system and the sink node. Afterwards, if other nodes satisfy the distance condition, the data is transmitted by the help of forwarder node or nodes. However, if the distance condition is not satisfied, the master node selects actor node or nodes, closest to midpoints of the distance between the sink node and the master node. Upon receiving the movement command, actor node or nodes move to these midpoints and the data packet is transmitted by the help of actor nodes. When the data transmission is completed, the actor nodes return to previous positions. By the way, in the system, the number of possible actor nodes is limited, so the TC-VBF algorithm can only work if the desired transmission distance is lower than the distance determined by the result of number of actor nodes times the threshold distance. Otherwise, the packet transmission will become harder.

Simulations done in this thesis show that the TC-VBF outperforms the VBF and the $\mathrm{HH}-\mathrm{VBF}$ in packet delivery ratio for both mid and long ranges. According to simulation results, nodes communicate within longer range with the TC-VBF algorithm. When average number of retransmission performances are investigated, the TC-VBF has lower retransmission values than the VBF and the HH-VBF. Average end-to-end delay performance of the TC-VBF is also as effective as that of the VBF and $\mathrm{HH}-\mathrm{VBF}$ for proximate distances. On the other hand, for long ranges, although end-to-end delay for the TC-VBF is quite high since node movements occur and it takes long time, transmission is successfully completed.

Consequently, when all simulations are analyzed, it is clearly stated that the TCVBF approach provides reliable packet transmission to the further distances for small networks, whereas currently used algorithms, the VBF and the HH-VBF, could not do with high successful transmission rates.

For further research, energy consumption of TC-VBF can be investigated and the transmission power control can be integrated into TC-VBF algorithm in order to reduce energy consumption. Furthermore, since actor nodes move in the TC-VBF algorithm, end-to-end delay is quite high. Hence, further research can be considered to decrease end-to-end delay. 


\section{REFERENCES}

[1] Partan, Jim, Jim Kurose, and Brian Neil Levine. "A survey of practical issues in underwater networks." ACM SIGMOBILE Mobile Computing and Communications Review 11.4 (2007): 23-33.

[2] Pompili, Dario, and Ian F. Akyildiz. "Overview of networking protocols for underwater wireless communications." Communications Magazine, IEEE 47.1 (2009): 97-102.

[3] Chitre, Mandar, Shiraz Shahabudeen, and Milica Stojanovic. "Underwater acoustic communications and networking: Recent advances and future challenges." Marine technology society journal 42.1 (2008): 103-116..

[4] Akyildiz, Ian F., Dario Pompili, and Tommaso Melodia. "Underwater acoustic sensor networks: research challenges." Ad hoc networks 3.3 (2005): 257-279.

[5] Otnes, Roald, et al. Underwater acoustic networking techniques. Springer Science \& Business Media, 2012.

[6] Hayajneh, Mohammad, Issa Khalil, and Yasser Gadallah. "An OFDMA-based MAC protocol for under water acoustic wireless sensor networks." Proceedings of the 2009 International Conference on Wireless Communications and Mobile Computing: Connecting the World Wirelessly. ACM, 2009.

[7] Akyildiz, Ian F., Dario Pompili, and Tommaso Melodia. "Challenges for efficient communication in underwater acoustic sensor networks." ACM Sigbed Review1.2 (2004): 3-8.

[8] Apostolas, C., R. Tafazolli, and B. G. Evans. "Comparison between elimination yield non pre-emptive priority multiple access (EY-NPMA) and dynamic TDMA (D-TDMA)." Personal, Indoor and Mobile Radio Communications, 1996. PIMRC'96., Seventh IEEE International Symposium on. Vol. 2. IEEE, 1996. 
[9] Pompili, Dario, Tommaso Melodia, and Ian F. Akyildiz. "A CDMA-based medium access control for underwater acoustic sensor networks." Wireless Communications, IEEE Transactions on 8.4 (2009): 1899-1909.

[10] Aliesawi, Salah, et al. "Performance comparison of IDMA receivers for underwater acoustic channels." Wireless Communication Systems (ISWCS), 2010 7th International Symposium on. IEEE, 2010.

[11] Abramson, Norman. "Development of the ALOHANET." Information Theory, IEEE Transactions on 31.2 (1985): 119-123.

[12] Roberts, Lawrence G. "ALOHA packet system with and without slots and capture." ACM SIGCOMM Computer Communication Review 5.2 (1975): 28-42.

[13] Karn, Phil. "MACA-a new channel access method for packet radio."ARRL/CRRL Amateur radio 9th computer networking conference. Vol. 140. 1990.

[14] Bharghavan, Vaduvur, et al. "MACAW: a media access protocol for wireless LAN's." ACM SIGCOMM Computer Communication Review. Vol. 24. No. 4. ACM, 1994.

[15] Ng, Hai-Heng, Wee-Seng Soh, and Mehul Motani. "MACA-U: A media access protocol for underwater acoustic networks." Global Telecommunications Conference, 2008. IEEE GLOBECOM 2008. IEEE. IEEE, 2008.

[16] Guo, Xiaoxing, Michael R. Frater, and Michael J. Ryan. "A propagation-delaytolerant collision avoidance protocol for underwater acoustic sensor networks."OCEANS 2006-Asia Pacific. IEEE, 2007.

[17] Guo, Xiaoxing, Michael R. Frater, and Michael J. Ryan. "Design of a propagation-delay-tolerant MAC protocol for underwater acoustic sensor networks." Oceanic Engineering, IEEE Journal of 34.2 (2009): 170-180.

[18] Peleato, Borja, and Milica Stojanovic. "Distance aware collision avoidance protocol for ad-hoc underwater acoustic sensor networks." Communications Letters, IEEE 11.12 (2007): 1025-1027.

[19] Liao, Wen-Hwa, and Chih-Chien Huang. "SF-MAC: A spatially fair MAC protocol for underwater acoustic sensor networks." Sensors Journal, IEEE 12.6 (2012): 1686-1694. 
[20] Haas, Zygmunt J., and Jing Deng. "Dual busy tone multiple access (DBTMA)-a multiple access control scheme for ad hoc networks." Communications, IEEE Transactions on 50.6 (2002): 975-985.

[21] Fullmer, Chane L., and J. J. Garcia-Luna-Aceves. Floor acquisition multiple access (FAMA) for packet-radio networks. Vol. 25. No. 4. ACM, 1995.

[21] Molins, Marcal, and Milica Stojanovic. "Slotted FAMA: a MAC protocol for underwater acoustic networks." OCEANS 2006-Asia Pacific. IEEE, 2007.

[23] Chen, Yin-Jun, and Hao-Li Wang. "Ordered CSMA: a collision-free MAC protocol for underwater acoustic networks." OCEANS 2007. IEEE, 2007.

[24] Noh, Youngtae, et al. "DOTS: A propagation Delay-aware Opportunistic MAC protocol for underwater sensor networks." Network Protocols (ICNP), 2010 18th IEEE International Conference on. IEEE, 2010.

[25] Ni, Jian, Bo Tan, and Rayadurgam Srikant. "Q-CSMA: queue-length-based CSMA/CA algorithms for achieving maximum throughput and low delay in wireless networks." Networking, IEEE/ACM Transactions on 20.3 (2012): 825-836.

[26] Park, Min Kyoung, and Volkan Rodoplu. "UWAN-MAC: An energy-efficient MAC protocol for underwater acoustic wireless sensor networks." Oceanic Engineering, IEEE Journal of 32.3 (2007): 710-720.

[27] Syed, Affan, Wei Ye, and John Heidemann. "T-Lohi: A new class of MAC protocols for underwater acoustic sensor networks." INFOCOM 2008. The 27th Conference on Computer Communications. IEEE. IEEE, 2008.

[28] Xie, Peng, and Jun-Hong Cui. "R-MAC: An energy-efficient MAC protocol for underwater sensor networks." Wireless Algorithms, Systems and Applications, 2007. WASA 2007. International Conference on. IEEE, 2007.

[29] Chirdchoo, Nitthita, Wee-seng Soh, and Kee Chaing Chua. "RIPT: A receiverinitiated reservation-based protocol for underwater acoustic networks."Selected Areas in Communications, IEEE Journal on 26.9 (2008): 1744-1753.

[30] Stojanovic, Milica. "Optimization of a data link protocol for an underwater acoustic channel." Oceans 2005-Europe. Vol. 1. IEEE, 2005.

[31] Doyle, Linda. "A multi-hop ARQ protocol for underwater acoustic networks." (2007). 
[32] Tomasi, Beatrice, et al. "A study of incremental redundancy hybrid ARQ over Markov channel models derived from experimental data." Proceedings of the Fifth ACM International Workshop on UnderWater Networks. ACM, 2010.

[33] Mo, Haining, et al. "Uw-harq: An underwater hybrid arq scheme: Design, implementation and initial test." Oceans, 2012. IEEE, 2012.

[34] Xie, Peng, and Jun-Hong Cui. "An FEC-based reliable data transport protocol for underwater sensor networks." Computer Communications and Networks, 2007. ICCCN 2007. Proceedings of 16th International Conference on. IEEE, 2007.

[35] Lee, Jae Won, Jin Yong Cheon, and Ho-Shin Cho. "A cooperative ARQ scheme in underwater acoustic sensor networks." OCEANS 2010 IEEE-Sydney. IEEE, 2010.

[36] Jacquet, Philippe, et al. "Optimized link state routing protocol for ad hoc networks." Multi Topic Conference, 2001. IEEE INMIC 2001. Technology for the 21st Century. Proceedings. IEEE International. IEEE, 2001.

[37] Perkins, Charles E., and Pravin Bhagwat. "Highly dynamic destinationsequenced distance-vector routing (DSDV) for mobile computers." ACM SIGCOMM computer communication review. Vol. 24. No. 4. ACM, 1994.

[38] Perkins, Charles, Elizabeth Belding-Royer, and Samir Das. "Ad hoc on-demand distance vector (AODV) routing”. No. RFC 3561. 2003.

[39] D.B. Johnson, A.M. Maltz, "Mobile Computing", Kluwer Academic Publishers, 1996

[40] Xie, Peng, Jun-Hong Cui, and Li Lao. "VBF: vector-based forwarding protocol for underwater sensor networks." Networking 2006. Networking technologies, services, and protocols; performance of computer and communication networks; mobile and wireless communications systems. Springer Berlin Heidelberg, 2006. 1216-1221.

[41] Nicolaou, Nicoletta, et al. "Improving the robustness of location-based routing for underwater sensor networks." OCEANS 2007-Europe. IEEE, 2007.

[42] Yan, Hai, Zhijie Jerry Shi, and Jun-Hong Cui. "DBR: depth-based routing for underwater sensor networks." NETWORKING 2008 Ad Hoc and Sensor Networks, 
Wireless Networks, Next Generation Internet. Springer Berlin Heidelberg, 2008. 7286.

[43] Ayaz, Muhammad, and Azween Abdullah. "Hop-by-hop dynamic addressing based (H2-DAB) routing protocol for underwater wireless sensor networks."Information and Multimedia Technology, 2009. ICIMT'09. International Conference on. IEEE, 2009.

[44] Jornet, Josep Miquel, Milica Stojanovic, and Michele Zorzi. "Focused beam routing protocol for underwater acoustic networks." Proceedings of the third ACM international workshop on Underwater Networks. ACM, 2008.

[45] Chen, Jinming, Xiaobing Wu, and Guihai Chen. "REBAR: A Reliable and Energy Balanced Routing Algorithm for UWSNs." Grid and Cooperative Computing, 2008. GCC'08. Seventh International Conference on. IEEE, 2008.

[46] Chirdchoo, Nitthita, Wee-Seng Soh, and Kee Chaing Chua. "Sector-based routing with destination location prediction for underwater mobile networks."Advanced Information Networking and Applications Workshops, 2009. WAINA'09. International Conference on. IEEE, 2009.

[47] Hwang, Daeyoup, and Dongkyun Kim. "DFR: Directional flooding-based routing protocol for underwater sensor networks." OCEANS 2008. IEEE, 2008.

[48] Carlson, Edward, Pierre-Philippe Beaujean, and Edgar An. "Location-aware routing protocol for underwater acoustic networks." OCEANS 2006. IEEE, 2006.

[49] Xu, Junfeng, et al. "Energy-efficient tree-based multipath power control for underwater sensor networks." Parallel and Distributed Systems, IEEE Transactions on 23.11 (2012): 2107-2116.

[50] Wang, Pu, Cheng Li, and Jun Zheng. "Distributed minimum-cost clustering protocol for underwater sensor networks (UWSNs)." Communications, 2007. ICC'07. IEEE International Conference on. IEEE, 2007.

[51] Domingo, Mari Carmen, and Rui Prior. "A distributed clustering scheme for underwater wireless sensor networks." Personal, Indoor and Mobile Radio Communications, 2007. PIMRC 2007. IEEE 18th International Symposium on. IEEE, 2007. 
[52] Santi, Paolo. "Topology control in wireless ad hoc and sensor networks." ACM computing surveys (CSUR) 37.2 (2005): 164-194.

[53] Li, Mo, Zhenjiang Li, and Athanasios V. Vasilakos. "A survey on topology control in wireless sensor networks: Taxonomy, comparative study, and open issues." Proceedings of the IEEE 101.12 (2013): 2538-2557.

[54] Tseng, Yu-Chee, Yen-Ning Chang, and Bour-Hour Tzeng. "Energy-efficient topology control for wireless ad hoc sensor networks." Journal of Information Science and Engineering 20.1 (2004): 27-37.

[55] Lim, Teck Lee, and G. Mohan. "Energy aware geographical routing and topology control to improve network lifetime in wireless sensor networks."Broadband Networks, 2005. BroadNets 2005. 2nd International Conference on. IEEE, 2005.

[56] Liu, Jilei, and Baochun Li. "Distributed topology control in wireless sensor networks with asymmetric links." Global Telecommunications Conference, 2003. GLOBECOM'03. IEEE. Vol. 3. IEEE, 2003.

[57] Wattenhofer, Roger, et al. "Distributed topology control for power efficient operation in multihop wireless ad hoc networks." INFOCOM 2001. Twentieth Annual Joint Conference of the IEEE Computer and Communications Societies. Proceedings. IEEE. Vol. 3. IEEE, 2001.

[58] Liu, Jilei, and Baochun Li. "Mobilegrid: capacity-aware topology control in mobile ad hoc networks." Computer Communications and Networks, 2002. Proceedings. Eleventh International Conference on. IEEE, 2002.

[59] Ding, Ling, et al. "Efficient algorithms for topology control problem with routing cost constraints in wireless networks." Parallel and Distributed Systems, IEEE Transactions on 22.10 (2011): 1601-1609.

[60] Costa, Paolo, et al. "A cooperative approach for topology control in wireless sensor networks: Experimental and simulation analysis." World of Wireless, Mobile and Multimedia Networks, 2008. WoWMoM 2008. 2008 International Symposium on a. IEEE, 2008. 
[61] Chen, Bai, Li-li Wang, and Yan-jin Ai. "Link interference prediction-based topology control algorithm for 3-D wireless sensor networks." Information Science and Engineering (ICISE), 2010 2nd International Conference on. IEEE, 2010.

[62] Liu, Linfeng. "A QoS-based topology control algorithm for underwater wireless sensor networks." International Journal of Distributed Sensor Networks 2010 (2010).

[63] Liu, Linfeng, Ruchuan Wang, and Fu Xiao. "Topology control algorithm for underwater wireless sensor networks using GPS-free mobile sensor nodes."Journal of Network and Computer Applications 35.6 (2012): 1953-1963.

[64] Liu, Linfeng, Ye Liu, and Ningshen Zhang. "A Complex Network Approach to Topology Control Problem in Underwater Acoustic Sensor Networks." Parallel and Distributed Systems, IEEE Transactions on 25.12 (2014): 3046-3055.

[65] Misra, Sudip, Tamoghna Ojha, and Ayan Mondal. "Game-Theoretic Topology Controlfor Opportunistic Localizationin Sparse Underwater Sensor Networks."Mobile Computing, IEEE Transactions on 14.5 (2015): 990-1003.

[66] Domingo, Mari Carmen. "Overview of channel models for underwater wireless communication networks." Physical Communication 1.3 (2008): 163-182.

[67] R.J. Urick, Principle of Underwater Sound, 3rd edition McGraw-Hill Inc., 1983

[68] Stojanovic, Milica, and James Preisig. "Underwater acoustic communication channels: Propagation models and statistical characterization."Communications Magazine, IEEE 47.1 (2009): 84-89.

[69] Waite, Ashley D., and A. D. Waite. Sonar for practising engineers. Vol. 3. London: Wiley, 2002.

[70] Sehgal, Anuj, Iyad Tumar, and Jürgen Schönwälder. "Aquatools: An underwater acoustic networking simulation toolkit." IEEE, Oceans, Sydney, Australia May(2010).

[71] Guerra, Federico, Paolo Casari, and Michele Zorzi. "World Ocean Simulation System (WOSS): a simulation tool for underwater networks with realistic propagation modeling." Proceedings of the Fourth ACM International Workshop on UnderWater Networks. ACM, 2009. 
[72] Xie, Peng, et al. "Aqua-Sim: an NS-2 based simulator for underwater sensor networks." OCEANS 2009, MTS/IEEE Biloxi-Marine Technology for Our Future: Global and Local Challenges. IEEE, 2009.

[73] Pan, Chengsheng, et al. "Modeling and simulation of channel for underwater communication network." Int. J. Innov. Comput. Inf. Control 8 (2012): 2149-2156. [74] Sozer, Ethem M., Milica Stojanovic, and John G. Proakis. "Underwater acoustic networks." Oceanic Engineering, IEEE Journal of 25.1 (2000): 72-83.

[75] Ovaliadis, Kyriakos, and Nick Savage. "Underwater Sensor Network Simulation Tool (USNeT)." International Journal of Computer Applications 71.22 (2013).

[76] Pompili, Dario, Tommaso Melodia, and Ian F. Akyildiz. "Deployment analysis in underwater acoustic wireless sensor networks." Proceedings of the 1st ACM international workshop on Underwater networks. ACM, 2006.

[77] Kilfoyle, Daniel B., and Arthur B. Baggeroer. "The state of the art in underwater acoustic telemetry." Oceanic Engineering, IEEE Journal of 25.1 (2000): 4-27. 